DEPARTMENT OF THE INTERIOR

U.S. GEOLOGICAL SURVEY

\title{
DATA REPORT FOR 1980-1981 SEISMIC-REFRACTION PROFILES IN THE WESTERN MOJAVE DESERT, CALIFORNIA
}

By

R. N. Harris, A. W. Walter and G. S. Fuis ${ }^{1}$

1988

Open-File Report 88-580

This report is preliminary and has not been reviewed for conformity with U.S. Geological Survey editorial standards and stratigraphic nomenclature. Any use of trade names is for descriptive purposes only and does not imply endorsement by the U.S. Geological Survey.

Menlo Park, California

1988

1 U.S. Geological Survey, Menlo Park, CA 


\section{CONTENTS}

Page

Introduction . . . . . . . . . . . . . . . . . . . . . . . . . . . . . . . . . 1

Instrumentation . . . . . . . . . . . . . . . . . . . . . . . . . . . . . 2

Data Processing and Editing . . . . . . . . . . . . . . . . . . . . . . . . . . . 2

Acknowledginents . . . . . . . . . . . . . . . . . . . . . . . . . . . . 3

References . . . . . . . . . . . . . . . . . . . . . . . . . . . . 3a

TABLES

TABLE 1. List of shots for western Mojave Desert, 1980-1981

2. Seismic recorder locations, western Mojave Desert, 1980-1981 . . . . . . . . 5

3. Explanation of columns in seismic recorder data table . . . . . . . . . . . . 14

4. Description of tape grade codes . . . . . . . . . . . . . . . . . . . . . 14

5. Seismic recorder data . . . . . . . . . . . . . . . . . . . . . . . . 15

\section{ILLUSTRATIONS}

Figure 1. Index map of study area . . . . . . . . . . . . . . . . . . . . . . . . . 28

2. Deployment 1 , shot numbers $1-5$. . . . . . . . . . . . . . . . . . . . . 29

3. Deployment 2, shot numbers 6-8 . . . . . . . . . . . . . . . . . . . . . 30

4. Deployment 3, shot number 9 . . . . . . . . . . . . . . . . . . . . . . 31

5. Deploynent 4, shot numbers 10-12 . . . . . . . . . . . . . . . . . . . . 32

6. Deployment 5, shotpoint 13 . . . . . . . . . . . . . . . . . . . . . . . 33

7. Record section for shot number 1 , shotpoint 4 , north of shotpoint 4 . . . . . . 34

8. Record section for shot number 1 , shotpoint 4 , south of shotpoint 4 . . . . . . 35

9. Record section for shot number 1 , shotpoint 4 , east-west recording array . . . . 36

10. Record section for shot number 2 , shotpoint 2 , north of shotpoint 2 . . . . . 37

11. Record section for shot number 2 , shotpoint 2 , south of shotpoint 2 . . . . . . 38

12. Record section for shot number 2 , shotpoint 2 , east-west recording array . . . 39

13. Record section for shot number 3, shotpoint 3, north-south recording array . . 40

14. Record section for shot number 3 , shotpoint 3 , west of shotpoint 3 . . . . . . . 41

15. Record section for shot number 3, shotpoint 3, east of shotpoint 3 . . . . . . . 42

16. Record section for shot number 4 , shotpoint 1 , north of shotpoint 2 . . . . . 43

17. Record section for shot number 4 , shotpoint 1 , south of shotpoint 2 . . . . . 44

18. Record section for shot number 4 , shotpoint 1 , west-east recording array . . . 45

19. Record section for shot number 5 , shotpoint 5 , north-south recording array . . 46

20. Record section for shot number 5 , shotpoint 5 , west of shotpoint 2 . . . . . . 47

21. Record section for shot number 5 , shotpoint 5 , east of shotpoint 2 . . . . . . 48 
22. Record section for shot number 6 , shotpoint 2 , south of shotpoint 2 . . . . . 49

23. Record section for shot number 6 , shotpoint 2 , north of shotpoint 7 . . . . . 50

24. Record section for shot number 7 , shotpoint 7 , north of shotpoint 7 . . . . . 51

25. Record section for shot number 7, shotpoint 7, north-south recording array . . 52

26. Record section for shot number 8 , shotpoint 6 , north of shotpoint 6 . . . . . 53

27. Record section for shot number 8 , shotpoint 6 , south of shotpoint 6 . . . . . 54

28. Record section for shot number 9, shotpoint 2, north of San Andreas Fault . . 55

29. Record section for shot number 9 , shotpoint 2 , western north-south recording array 56

30. Record section for shot number 9, shotpoint 2, south of San Andreas Fault . . 57

31. Record section for shot number 9 , shotpoint 2 , east of shotpoint 7 . . . . . . 58

32. Record section for shot number 10 , shotpoint 10 , north of shotpoint 10 . . . . $\quad 59$

33. Record section for shot number 10 , shotpoint 10 , south of shotpoint 10 . . . . 60

34. Record section for shot number 11 , shotpoint 11 , north of shotpoint 11 . . . . 61

35. Record section for shot number 11, shotpoint 11, south of shotpoint 11 . . . . 62

36. Record section for shot number 12 , shotpoint 12 , northern half . . . . . . . 63

37. Record section for shot number 12 , shotpoint 12 , southern half . . . . . . . 64

38. Record section for shot number 13 , shotpoint 13 . . . . . . . . . . . . 65 


\section{INTRODUCTION}

During 1980 and 1981, the U.S. Geological Survey collected seismic refraction data in the western Mojave Desert (fig. 1). The seismic refraction data extends from north of the Garlock Fault, south through the Mojave Desert, and across the San Andreas Fault into the San Gabriel Mountains. This report describes and documents the recorded data, which was collected over three field sessions. In total thirteen shots were fired at 11 different shotpoints (Table 1). The experiment consisted of five deployments of a set of 100 portable seismic recorders, during which seismic energy from one or more shots was recorded. The data set was acquired with five deployments of a set of 100 portable seismic recorders.

The first phase (deployments 1 - 3; figs. 2 - 4), in June 1980, was designed to investigate P-wave anisotropic-velocity using a south trending profile with two cross-arrays. Station spacing for these deployments was about $0.5 \mathrm{~km}$. The U.S. Geological Survey loaded and detonated all shots in the first phase.

The second phase of the experiment (deployments 4 and 5; figs. 5 and 6), in April and November 1981, recorded two quarry blasts (shots 10 and 11) and a missile detonation (shot 12) so that the profile from phase one could be extened to the north and south. Station spacing for these deployments was about $1.5 \mathrm{~km}$. During this phase of the experiment some of the original recording sites were reoccuppied. Given and Koesterer (1983) describe shot number 13, which was detonated on Santa Catalina island.

Shot times and shotpoint locations are presented in table 1. Geographic locations for each recording site are in table 2 . The location of each recording site relative to the shot point and information about each data recording for that site is provided in table 3. Seismic record sections are plotted in figures $7-38$. The data is stored on magnetic tape in SEGY format, described by Barry and others (1975), and may be obtained by writing the National

Geophysical Data Center, National Oceanic and Atmospheric Administration, 325 Broadway, Boulder CO, 80303. 
On the basis of this data and in-situ stress measurements Kohler and Healy (1981) suggest that $\mathrm{P}$-wave anisotropic velocity arises from the action of tectonic stress on highangle macro-factures. Fuis and others (1986) modeled the crust along the north - south profile between shotpoint 12 and the San Gabriel Mountains. Other work in the area includes six deep seismic reflection profiles collected by the Consortium for Continental Reflection Profiling in 1982 (Cheadle and others, 1986).

\section{INSTRUMENTATION}

Each seismic refraction recorder consists of a $2-\mathrm{Hz}$ vertical component seismometer coupled to an analog tape cassette recording system (Healy and others, 1982) The electronics, including signal response, calibration and diagnostic tests is described by Murphy (1988).

\section{DATA PROCESSING AND EDITING}

For each shot, 20 seconds of seismic signal from playback of cassette data tapes were digitized at 200 samples/sec. The digitizing start time $(T)$ is calculated from:

$$
T=S-2 \sec +X / R+C
$$

$$
\begin{aligned}
& \text { where } \\
& \mathrm{S}=\text { shot time (Table } 1) \\
& \mathrm{X}=\text { shot to recorder distance in } \mathrm{km} \text { (Table } 2), \\
& \mathrm{R}=\text { reduction velocity in } \mathrm{km} / \mathrm{sec}, \\
& \mathrm{C}=\text { recorder clock drift in seconds. }
\end{aligned}
$$

Recorder and shotpoint locations were determined from USGS 7.5' and $15^{\prime}$ topographic maps and are estimated to be accurate to 10 meters. Shotpoint to recorder distances are calculated 
with the algorithm of Sodano and Robinson (1963). The reduction velocity for all shots except shotpoint 13 is $6.0 \mathrm{~km} / \mathrm{sec}$; a reduction velocity of $8.0 \mathrm{~km} / \mathrm{sec}$ was used for shotpoint 13. The clock drift of each recorder was checked before and after each deployment and was assumed to be linear. Estimated drifts at shot time were usually less than 50 milliseconds.

Record sections, shown in figures $7-38$, are plotted with the maximum anplitude for each trace normalized to a common value. Distances are relative to the shotpoint, except as noted, and distances to the west and north of the shotpoint are given negative values.

\section{ACKNOWLEDGMENTS}

This experiment was initiated by $\mathrm{J}$. Healy. We wish to express our appreciation to $\mathrm{J}$. Bonomolo, R. Catchings, S. Gallanthine, L. Hoffman, W. Kohler, B. Lutter, J. Roloff, L. Sutton, A. Young for help with the field operations. 


\section{REFERENCES}

Barry, K. M., Cavers, D. A., and Kneale, C. W., 1975, Recommended standards for digital tape formats, Geophysics 40, 344-352 p.

Cheadle, M. J., Czuchra, B. L., Byrne, T., Ando, C. J., Oliver, J. E., Brown, L. D., Kaufman, S., Malin, P. E., and Phinney, R. A., 1986, The deep crustal structure of the Mojave Desert, California, from COCORP seismic reflection data, Tectonics, vol. 5, no. 2, p. 293-320.

Fuis, G. S., A. W. Walter, W. D. Mooney, and J. McCarthy, 1986, Crustal velocity structure of the Salton Trough and western Mojave Desert from seismic refraction measurements, GSA Abstracts with Programs, Cordilleran section, v. 18, no. 2 , p. 107.

Given, Douglas, D., and Koesterer, Charles, L., 1983, Station arrival data for a quarry blast on Santa Catalina Island, California: U.S. Geological Survey Open-File Report 83-462, 12 p.

Healy, J. H., Mooney, W. D., Blank, H. R., Gettings, M. E., Kohler, W. M., Lamson, R. J., Leone, L. E., 1982, Saudi Arabian seismic deep-refraction profile: Final project report: U.S. Geological Survey Open-File Report 02-37, 429 p.

Kohler, W. M., and Healy, J., H., 1981, The mechanism of anisotrpic wave velocity in crystalline rocks of the Mojave Desert (abs.): Eos, American Geophysical Union Transactions, v. 62, no. 45, p. 961.

Murphy, J., 1988, USGS FM cassette seismic-recording system: U.S. Geological Suvey Open-File Report (in press). 
TABLE 1.-List of Shots for western Mojave Desert, 1980-1981

\begin{tabular}{|c|c|c|c|c|c|c|c|c|c|c|}
\hline $\begin{array}{l}\text { Deployment } \\
\text { Number }\end{array}$ & $\begin{array}{c}\text { Shot } \\
\text { Number }\end{array}$ & $\begin{array}{l}\text { Shot } \\
\text { Point }\end{array}$ & & Dete & $\begin{array}{c}\text { Shot } \\
\text { (Day, Hour, Min, Sec) }\end{array}$ & & $\begin{array}{l}\text { etitude } \\
\text { eg, min) }\end{array}$ & $\begin{array}{l}\text { Longitude } \\
\text { (deg, min) }\end{array}$ & $\begin{array}{l}\text { Elevation } \\
\text { (meters) }\end{array}$ & $\begin{array}{l}\text { Size } \\
(\mathbf{k g})\end{array}$ \\
\hline 1 & 1 & 4 & JUN & 15,1980 & $167: 06: 00: 00.010$ & 34 & 50.0285 & 11739.3073 & 908 & 470 \\
\hline 1 & 2 & 2 & JUN & 15,1980 & $167: 06: 29: 60.003$ & 34 & 48.0542 & 11746.7844 & 780 & 358 \\
\hline 1 & 3 & 3 & JUN & 15,1980 & $167: 07: 00: 00.008$ & 34 & 57.0705 & 11745.8702 & 713 & 381 \\
\hline 1 & 4 & 1 & JUN & 15,1980 & $167: 07: 29: 59.989$ & 34 & 47.5793 & 11752.9454 & 704 & 289 \\
\hline 1 & 5 & 5 & JUN & 15,1980 & $167: 08: 30: 00.010$ & 34 & 42.9739 & 11746.7313 & 915 & 358 \\
\hline 2 & 6 & 2 & JUN & 25,1980 & $177: 06: 00: 00.012$ & 34 & 48.0542 & 11746.7844 & 780 & 381 \\
\hline 2 & 7 & 7 & JUN & 25,1980 & $177: 07: 00: 00.012$ & 34 & 27.8142 & 11751.6504 & 1073 & 381 \\
\hline 2 & 8 & 6 & JUN & 25,1980 & $177: 08: 00: 00.016$ & 34 & 32.6843 & 11751.2842 & 882 & 142 \\
\hline 3 & 9 & 2 & JUN & 28,1980 & $180: 08: 00: 00.016$ & 34 & 48.0542 & 11746.7844 & 780 & 970 \\
\hline 4 & 10 & 10 & APR & 24,1981 & $114: 20: 01: 13.785$ & 35 & 2.6402 & 11740.6659 & 770 & 13,996 \\
\hline 4 & 11 & 11 & APR & 24,1981 & $114: 20: 02: 23.085$ & 35 & 2.7052 & 11740.8376 & 771 & 3,255 \\
\hline 4 & 12 & 12 & APR & 25,1981 & $115: 21: 00: 58.487$ & 35 & 42.5200 & 11731.8600 & 753 & 37,324 \\
\hline 5 & 13 & 13 & NOV & 8,1981 & $312: 20: 00: 00.190$ & 33 & 19.0307 & 11818.3825 & 55 & 67,183 \\
\hline
\end{tabular}


TABLE 2.-Seismic Recorder Locations, western Mojave Desert, 1980-1981

\begin{tabular}{|c|c|c|c|}
\hline $\begin{array}{l}\text { Station } \\
\text { number }\end{array}$ & $\begin{array}{l}\text { Latitude } \\
\text { deg min }\end{array}$ & $\begin{array}{l}\text { Longitude } \\
\text { deg min }\end{array}$ & $\begin{array}{c}\text { Elevation } \\
\text { meters }\end{array}$ \\
\hline $\begin{array}{l}101 \\
102 \\
103 \\
104 \\
105 \\
106 \\
107 \\
108 \\
109 \\
110\end{array}$ & $\begin{array}{ll}34 & 57.04 \\
34 & 56.48 \\
34 & 56.11 \\
34 & 55.73 \\
34 & 55.27 \\
34 & 54.74 \\
34 & 54.45 \\
34 & 54.24 \\
34 & 54.04 \\
34 & 53.90\end{array}$ & $\begin{array}{ll}117 & 45.84 \\
117 & 46.09 \\
117 & 45.74 \\
117 & 45.50 \\
117 & 45.56 \\
117 & 45.39 \\
117 & 45.41 \\
117 & 45.41 \\
117 & 45.77 \\
117 & 46.02\end{array}$ & $\begin{array}{l}713 \\
733 \\
777 \\
755 \\
719 \\
724 \\
727 \\
728 \\
721 \\
715\end{array}$ \\
\hline $\begin{array}{l}111 \\
112 \\
113 \\
114 \\
115 \\
116 \\
117 \\
118 \\
119 \\
121\end{array}$ & $\begin{array}{ll}34 & 53.73 \\
34 & 53.42 \\
34 & 53.14 \\
34 & 52.76 \\
34 & 52.56 \\
34 & 52.28 \\
34 & 52.03 \\
34 & 51.73 \\
34 & 51.46 \\
34 & 50.04\end{array}$ & $\begin{array}{ll}117 & 46.34 \\
117 & 46.32 \\
117 & 46.34 \\
117 & 46.34 \\
117 & 46.36 \\
117 & 46.35 \\
117 & 46.36 \\
117 & 46.36 \\
117 & 46.35 \\
117 & 39.30\end{array}$ & $\begin{array}{l}711 \\
715 \\
718 \\
722 \\
725 \\
728 \\
735 \\
739 \\
738 \\
908\end{array}$ \\
\hline $\begin{array}{l}122 \\
123 \\
124 \\
125 \\
126 \\
127 \\
128 \\
129 \\
130\end{array}$ & $\begin{array}{ll}34 & 49.84 \\
34 & 49.88 \\
34 & 49.92 \\
34 & 49.97 \\
34 & 50.07 \\
34 & 50.12 \\
34 & 50.17 \\
34 & 50.22 \\
34 & 50.29\end{array}$ & $\begin{array}{ll}117 & 37.78 \\
117 & 38.05 \\
117 & 38.40 \\
117 & 38.75 \\
117 & 39.43 \\
117 & 39.77 \\
117 & 40.13 \\
117 & 40.47 \\
117 & 40.80\end{array}$ & $\begin{array}{l}957 \\
954 \\
939 \\
927 \\
902 \\
890 \\
887 \\
881 \\
875\end{array}$ \\
\hline $\begin{array}{l}131 \\
132 \\
133 \\
134 \\
135 \\
136 \\
137 \\
138 \\
139 \\
141\end{array}$ & $\begin{array}{ll}34 & 50.25 \\
34 & 50.33 \\
34 & 50.41 \\
34 & 50.47 \\
34 & 50.55 \\
34 & 50.50 \\
34 & 50.44 \\
34 & 50.39 \\
34 & 50.32 \\
34 & 42.99\end{array}$ & $\begin{array}{ll}117 & 41.15 \\
117 & 41.48 \\
117 & 41.84 \\
117 & 42.17 \\
117 & 42.48 \\
117 & 42.81 \\
117 & 43.16 \\
117 & 43.49 \\
117 & 43.87 \\
117 & 46.73\end{array}$ & $\begin{array}{l}860 \\
860 \\
847 \\
841 \\
829 \\
820 \\
811 \\
792 \\
792 \\
915\end{array}$ \\
\hline
\end{tabular}


TABLE 2.-Seismic Recorder Locations, western Mojave Desert, 1980-1981-Continued

\begin{tabular}{|c|c|c|c|}
\hline $\begin{array}{l}\text { Station } \\
\text { number }\end{array}$ & $\begin{array}{l}\text { Latitude } \\
\text { deg min }\end{array}$ & $\begin{array}{l}\text { Longitude } \\
\text { deg min }\end{array}$ & $\begin{array}{c}\text { Elevation } \\
\text { meters }\end{array}$ \\
\hline $\begin{array}{l}142 \\
143 \\
144 \\
145 \\
146 \\
147 \\
148 \\
149 \\
150 \\
151\end{array}$ & $\begin{array}{ll}34 & 43.20 \\
34 & 43.51 \\
34 & 43.80 \\
34 & 44.09 \\
34 & 44.40 \\
34 & 44.68 \\
34 & 44.98 \\
34 & 45.28 \\
34 & 45.57 \\
34 & 45.86\end{array}$ & $\begin{array}{ll}117 & 46.41 \\
117 & 46.41 \\
117 & 46.40 \\
117 & 46.41 \\
117 & 46.41 \\
117 & 46.40 \\
117 & 46.40 \\
117 & 46.41 \\
117 & 46.41 \\
117 & 46.41\end{array}$ & $\begin{array}{l}913 \\
919 \\
925 \\
928 \\
933 \\
936 \\
927 \\
933 \\
939 \\
942\end{array}$ \\
\hline $\begin{array}{l}152 \\
153 \\
154 \\
155 \\
156 \\
157 \\
158 \\
159 \\
160 \\
161\end{array}$ & $\begin{array}{ll}34 & 46.16 \\
34 & 46.45 \\
34 & 46.75 \\
34 & 47.03 \\
34 & 47.32 \\
34 & 47.61 \\
34 & 47.92 \\
34 & 48.21 \\
34 & 48.50 \\
34 & 47.56\end{array}$ & $\begin{array}{ll}117 & 46.41 \\
117 & 46.41 \\
117 & 46.42 \\
117 & 46.42 \\
117 & 46.42 \\
117 & 46.42 \\
117 & 46.42 \\
117 & 46.42 \\
117 & 46.42 \\
117 & 52.94\end{array}$ & $\begin{array}{l}951 \\
954 \\
933 \\
902 \\
872 \\
847 \\
826 \\
811 \\
796 \\
704\end{array}$ \\
\hline $\begin{array}{l}162 \\
163 \\
164 \\
165 \\
166 \\
167 \\
168 \\
169 \\
170 \\
171\end{array}$ & $\begin{array}{ll}34 & 48.47 \\
34 & 48.47 \\
34 & 48.47 \\
34 & 48.47 \\
34 & 48.47 \\
34 & 48.47 \\
34 & 48.48 \\
34 & 48.48 \\
34 & 48.48 \\
34 & 48.49\end{array}$ & $\begin{array}{ll}117 & 53.86 \\
117 & 53.50 \\
117 & 53.14 \\
117 & 52.80 \\
117 & 52.44 \\
117 & 52.08 \\
117 & 51.74 \\
117 & 51.37 \\
117 & 51.03 \\
117 & 50.64\end{array}$ & $\begin{array}{l}704 \\
703 \\
703 \\
702 \\
701 \\
701 \\
700 \\
700 \\
701 \\
700\end{array}$ \\
\hline $\begin{array}{l}172 \\
173 \\
174 \\
175 \\
176 \\
177 \\
178 \\
179 \\
180 \\
181\end{array}$ & $\begin{array}{ll}34 & 48.49 \\
34 & 48.49 \\
34 & 48.48 \\
34 & 48.49 \\
34 & 48.49 \\
34 & 48.49 \\
34 & 48.49 \\
34 & 48.49 \\
34 & 48.50 \\
34 & 48.04\end{array}$ & $\begin{array}{ll}117 & 50.29 \\
117 & 49.96 \\
117 & 49.59 \\
117 & 49.27 \\
117 & 48.90 \\
117 & 48.54 \\
117 & 48.18 \\
117 & 47.84 \\
117 & 47.46 \\
117 & 46.78\end{array}$ & $\begin{array}{l}704 \\
709 \\
719 \\
725 \\
735 \\
716 \\
725 \\
765 \\
774 \\
780\end{array}$ \\
\hline
\end{tabular}


TABLE 2.-Seismic Recorder Locations, western Mojave Desert, 1980-1981-Continued

\begin{tabular}{|c|c|c|c|}
\hline $\begin{array}{l}\text { Station } \\
\text { number }\end{array}$ & $\begin{array}{l}\text { Latitude } \\
\text { deg min }\end{array}$ & $\begin{array}{l}\text { Longitude } \\
\text { deg min }\end{array}$ & $\begin{array}{c}\text { Elevation } \\
\text { meters }\end{array}$ \\
\hline $\begin{array}{l}182 \\
183 \\
184 \\
185 \\
186 \\
187 \\
188 \\
189 \\
190 \\
191\end{array}$ & $\begin{array}{ll}34 & 48.81 \\
34 & 49.09 \\
34 & 49.38 \\
34 & 48.50 \\
34 & 48.50 \\
34 & 50.27 \\
34 & 50.19 \\
34 & 50.14 \\
34 & 50.07 \\
34 & 50.01\end{array}$ & $\begin{array}{ll}117 & 46.41 \\
117 & 46.41 \\
117 & 46.41 \\
117 & 46.75 \\
117 & 47.10 \\
117 & 44.17 \\
117 & 44.51 \\
117 & 44.85 \\
117 & 45.19 \\
117 & 45.53\end{array}$ & $\begin{array}{l}780 \\
777 \\
774 \\
789 \\
780 \\
792 \\
786 \\
783 \\
780 \\
777\end{array}$ \\
\hline $\begin{array}{l}192 \\
193 \\
194 \\
195 \\
196 \\
197 \\
198 \\
199 \\
200 \\
201\end{array}$ & $\begin{array}{ll}34 & 49.96 \\
34 & 49.90 \\
34 & 49.87 \\
34 & 49.58 \\
34 & 50.16 \\
34 & 50.28 \\
34 & 50.57 \\
34 & 50.85 \\
34 & 51.16 \\
34 & 39.66\end{array}$ & $\begin{array}{ll}117 & 45.87 \\
117 & 46.22 \\
117 & 46.40 \\
117 & 46.40 \\
117 & 46.42 \\
117 & 46.42 \\
117 & 46.42 \\
117 & 46.42 \\
117 & 46.43 \\
117 & 47.08\end{array}$ & $\begin{array}{l}774 \\
768 \\
762 \\
771 \\
756 \\
753 \\
750 \\
747 \\
741 \\
846\end{array}$ \\
\hline $\begin{array}{l}202 \\
203 \\
204 \\
205 \\
206 \\
207 \\
208 \\
209 \\
210 \\
211\end{array}$ & $\begin{array}{ll}34 & 39.65 \\
34 & 39.36 \\
34 & 39.07 \\
34 & 38.79 \\
34 & 38.76 \\
34 & 38.76 \\
34 & 38.75 \\
34 & 38.47 \\
34 & 38.18 \\
34 & 37.86\end{array}$ & $\begin{array}{ll}117 & 47.44 \\
117 & 47.45 \\
117 & 47.45 \\
117 & 47.44 \\
117 & 47.78 \\
117 & 48.13 \\
117 & 48.49 \\
117 & 48.50 \\
117 & 48.50 \\
117 & 48.51\end{array}$ & $\begin{array}{l}850 \\
828 \\
812 \\
808 \\
803 \\
799 \\
794 \\
796 \\
799 \\
802\end{array}$ \\
\hline $\begin{array}{l}212 \\
213 \\
214 \\
215 \\
216 \\
217 \\
218 \\
219 \\
220 \\
221\end{array}$ & $\begin{array}{ll}34 & 37.58 \\
34 & 37.31 \\
34 & 36.97 \\
34 & 36.67 \\
34 & 36.40 \\
34 & 36.13 \\
34 & 35.83 \\
34 & 35.58 \\
34 & 35.27 \\
34 & 34.93\end{array}$ & $\begin{array}{ll}117 & 48.51 \\
117 & 48.50 \\
117 & 48.49 \\
117 & 48.49 \\
117 & 48.49 \\
117 & 48.49 \\
117 & 48.50 \\
117 & 48.49 \\
117 & 48.49 \\
117 & 48.48\end{array}$ & $\begin{array}{l}805 \\
808 \\
813 \\
817 \\
820 \\
823 \\
828 \\
832 \\
837 \\
843\end{array}$ \\
\hline
\end{tabular}


TABLE 2.-Seismic Recorder Locations, western Mojave Desert, 1980-1981-Continued

\begin{tabular}{|c|c|c|c|}
\hline $\begin{array}{l}\text { Station } \\
\text { number }\end{array}$ & $\begin{array}{l}\text { Latitude } \\
\text { deg min }\end{array}$ & $\begin{array}{l}\text { Longitude } \\
\text { deg min }\end{array}$ & $\begin{array}{c}\text { Elevation } \\
\text { meters }\end{array}$ \\
\hline $\begin{array}{l}222 \\
223 \\
224 \\
225 \\
226 \\
227 \\
228 \\
229 \\
230 \\
231\end{array}$ & $\begin{array}{ll}34 & 34.67 \\
34 & 34.37 \\
34 & 34.05 \\
34 & 33.80 \\
34 & 33.52 \\
34 & 33.21 \\
34 & 32.91 \\
34 & 32.53 \\
34 & 32.25 \\
34 & 31.96\end{array}$ & $\begin{array}{ll}117 & 48.48 \\
117 & 48.48 \\
117 & 48.49 \\
117 & 48.47 \\
117 & 48.47 \\
117 & 48.52 \\
117 & 48.49 \\
117 & 48.50 \\
117 & 48.50 \\
117 & 48.51\end{array}$ & $\begin{array}{l}847 \\
853 \\
860 \\
866 \\
872 \\
878 \\
884 \\
893 \\
902 \\
911\end{array}$ \\
\hline $\begin{array}{l}232 \\
233 \\
234 \\
235 \\
236 \\
237 \\
238 \\
239 \\
240 \\
248\end{array}$ & $\begin{array}{ll}34 & 31.69 \\
34 & 32.53 \\
34 & 32.53 \\
34 & 32.53 \\
34 & 32.54 \\
34 & 32.55 \\
34 & 32.54 \\
34 & 32.55 \\
34 & 32.70 \\
34 & 42.90\end{array}$ & 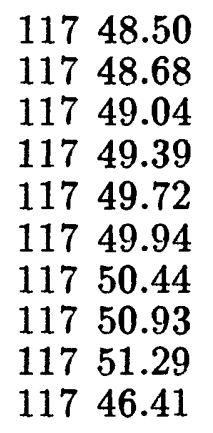 & $\begin{array}{l}922 \\
893 \\
892 \\
890 \\
890 \\
890 \\
890 \\
887 \\
882 \\
908\end{array}$ \\
\hline $\begin{array}{l}249 \\
250 \\
251 \\
252 \\
253 \\
254 \\
255 \\
256 \\
257 \\
258\end{array}$ & $\begin{array}{ll}34 & 42.61 \\
34 & 42.30 \\
34 & 42.01 \\
34 & 41.72 \\
34 & 41.45 \\
34 & 41.13 \\
34 & 40.86 \\
34 & 40.56 \\
34 & 40.28 \\
34 & 40.02\end{array}$ & $\begin{array}{ll}117 & 46.41 \\
117 & 46.41 \\
117 & 46.39 \\
117 & 46.39 \\
117 & 46.40 \\
117 & 46.40 \\
117 & 46.39 \\
117 & 46.43 \\
117 & 46.39 \\
117 & 46.40\end{array}$ & $\begin{array}{l}908 \\
908 \\
899 \\
898 \\
891 \\
881 \\
914 \\
881 \\
864 \\
852\end{array}$ \\
\hline $\begin{array}{l}259 \\
260 \\
261 \\
262 \\
263 \\
264 \\
265 \\
266 \\
267 \\
268\end{array}$ & $\begin{array}{ll}34 & 39.67 \\
34 & 39.66 \\
34 & 31.31 \\
34 & 31.25 \\
34 & 31.08 \\
34 & 30.80 \\
34 & 30.57 \\
34 & 30.36 \\
34 & 30.06 \\
34 & 29.82\end{array}$ & $\begin{array}{ll}117 & 46.39 \\
117 & 46.74 \\
117 & 48.49 \\
117 & 48.53 \\
117 & 48.54 \\
117 & 48.52 \\
117 & 48.51 \\
117 & 48.82 \\
117 & 49.03 \\
117 & 49.02\end{array}$ & $\begin{array}{r}837 \\
838 \\
939 \\
945 \\
956 \\
963 \\
969 \\
975 \\
991 \\
1006\end{array}$ \\
\hline
\end{tabular}


TABLE 2.-Seismic Recorder Locations, western Mojave Desert, 1980-1981-Continued

\begin{tabular}{|c|c|c|c|}
\hline $\begin{array}{l}\text { Station } \\
\text { number }\end{array}$ & $\begin{array}{l}\text { Latitude } \\
\text { deg min }\end{array}$ & $\begin{array}{l}\text { Longitude } \\
\text { deg min }\end{array}$ & $\begin{array}{c}\text { Elevation } \\
\text { meters }\end{array}$ \\
\hline $\begin{array}{l}269 \\
270 \\
271 \\
272 \\
273 \\
274 \\
275 \\
276 \\
277 \\
278\end{array}$ & $\begin{array}{ll}34 & 29.46 \\
34 & 29.17 \\
34 & 29.16 \\
34 & 29.16 \\
34 & 29.16 \\
34 & 28.86 \\
34 & 28.56 \\
34 & 27.92 \\
34 & 27.61 \\
34 & 27.22\end{array}$ & $\begin{array}{ll}117 & 49.02 \\
117 & 49.03 \\
117 & 49.36 \\
117 & 49.73 \\
117 & 49.99 \\
117 & 50.06 \\
117 & 50.06 \\
117 & 50.06 \\
117 & 50.06 \\
117 & 49.88\end{array}$ & $\begin{array}{l}1018 \\
1036 \\
1036 \\
1036 \\
1030 \\
1055 \\
1073 \\
1140 \\
1201 \\
1302\end{array}$ \\
\hline $\begin{array}{l}279 \\
280 \\
281 \\
282 \\
283 \\
284 \\
285 \\
286 \\
287 \\
288\end{array}$ & $\begin{array}{ll}34 & 28.40 \\
34 & 27.81 \\
34 & 27.93 \\
34 & 28.05 \\
34 & 28.12 \\
34 & 28.09 \\
34 & 28.20 \\
34 & 26.89 \\
34 & 26.62 \\
34 & 26.51\end{array}$ & $\begin{array}{ll}117 & 50.34 \\
117 & 51.65 \\
117 & 51.52 \\
117 & 51.29 \\
117 & 51.19 \\
117 & 50.85 \\
117 & 50.55 \\
117 & 49.98 \\
117 & 50.06 \\
117 & 50.07\end{array}$ & $\begin{array}{l}1073 \\
1073 \\
1073 \\
1073 \\
1073 \\
1109 \\
1097 \\
1158 \\
1158 \\
1170\end{array}$ \\
\hline $\begin{array}{l}289 \\
290 \\
291 \\
292 \\
293 \\
294 \\
295 \\
296 \\
297 \\
298\end{array}$ & $\begin{array}{ll}34 & 26.17 \\
34 & 25.86 \\
34 & 25.61 \\
34 & 25.35 \\
34 & 25.02 \\
34 & 24.92 \\
34 & 24.82 \\
34 & 24.65 \\
34 & 24.35 \\
34 & 24.10\end{array}$ & $\begin{array}{ll}117 & 50.10 \\
117 & 50.06 \\
117 & 50.20 \\
117 & 50.27 \\
117 & 50.19 \\
117 & 49.86 \\
117 & 49.54 \\
117 & 49.30 \\
117 & 49.33 \\
117 & 49.37\end{array}$ & $\begin{array}{l}1186 \\
1207 \\
1219 \\
1231 \\
1256 \\
1280 \\
1292 \\
1317 \\
1341 \\
1353\end{array}$ \\
\hline $\begin{array}{l}300 \\
301 \\
302 \\
303 \\
304 \\
305 \\
306 \\
307 \\
308\end{array}$ & $\begin{array}{ll}34 & 23.63 \\
34 & 30.27 \\
34 & 29.60 \\
34 & 29.27 \\
34 & 28.64 \\
34 & 28.15 \\
34 & 27.50 \\
34 & 26.91 \\
34 & 26.43\end{array}$ & $\begin{array}{ll}117 & 49.21 \\
117 & 53.75 \\
117 & 53.74 \\
117 & 53.75 \\
117 & 53.98 \\
117 & 53.92 \\
117 & 53.97 \\
117 & 53.97 \\
117 & 53.74\end{array}$ & $\begin{array}{r}1402 \\
933 \\
984 \\
1012 \\
1058 \\
1097 \\
1170 \\
1183 \\
1218\end{array}$ \\
\hline
\end{tabular}


TABLE 2.-Seismic Recorder Locations, western Mojave Desert, 1980-1981-Continued

\begin{tabular}{|c|c|c|c|}
\hline $\begin{array}{l}\text { Station } \\
\text { number }\end{array}$ & $\begin{array}{l}\text { Latitude } \\
\text { deg min }\end{array}$ & $\begin{array}{l}\text { Longitude } \\
\text { deg min }\end{array}$ & $\begin{array}{c}\text { Elevation } \\
\text { meters }\end{array}$ \\
\hline $\begin{array}{l}309 \\
310 \\
311 \\
312 \\
313 \\
314 \\
315 \\
316 \\
317 \\
318\end{array}$ & $\begin{array}{ll}34 & 25.89 \\
34 & 25.32 \\
34 & 24.91 \\
34 & 27.45 \\
34 & 27.38 \\
34 & 27.39 \\
34 & 26.80 \\
34 & 26.43 \\
34 & 25.88 \\
34 & 25.68\end{array}$ & $\begin{array}{ll}117 & 53.82 \\
117 & 53.92 \\
117 & 53.90 \\
117 & 53.40 \\
117 & 52.60 \\
117 & 51.70 \\
117 & 51.11 \\
117 & 50.62 \\
117 & 49.53 \\
117 & 49.04\end{array}$ & $\begin{array}{l}1292 \\
1378 \\
1451 \\
1161 \\
1195 \\
1091 \\
1127 \\
1161 \\
1231 \\
1298\end{array}$ \\
\hline $\begin{array}{l}319 \\
320 \\
321 \\
322 \\
323 \\
324 \\
325 \\
326 \\
327 \\
328\end{array}$ & $\begin{array}{ll}34 & 25.76 \\
34 & 25.38 \\
34 & 14.29 \\
34 & 14.47 \\
34 & 14.94 \\
34 & 15.13 \\
34 & 15.36 \\
34 & 15.60 \\
34 & 15.80 \\
34 & 16.29\end{array}$ & $\begin{array}{ll}117 & 48.52 \\
117 & 48.08 \\
117 & 51.74 \\
117 & 51.88 \\
117 & 51.80 \\
117 & 51.55 \\
117 & 51.46 \\
117 & 51.21 \\
117 & 50.61 \\
117 & 50.78\end{array}$ & $\begin{array}{r}1396 \\
1451 \\
475 \\
482 \\
543 \\
570 \\
561 \\
628 \\
695 \\
756\end{array}$ \\
\hline $\begin{array}{l}329 \\
330 \\
331 \\
332 \\
333 \\
334 \\
335 \\
336 \\
337 \\
338\end{array}$ & $\begin{array}{ll}34 & 16.68 \\
34 & 16.81 \\
34 & 16.97 \\
34 & 17.24 \\
34 & 17.58 \\
34 & 17.98 \\
34 & 18.21 \\
34 & 18.54 \\
34 & 18.82 \\
34 & 19.12\end{array}$ & $\begin{array}{ll}117 & 50.68 \\
117 & 50.31 \\
117 & 50.55 \\
117 & 50.48 \\
117 & 49.96 \\
117 & 50.04 \\
117 & 50.70 \\
117 & 50.41 \\
117 & 49.99 \\
117 & 50.37\end{array}$ & $\begin{array}{r}817 \\
902 \\
939 \\
1006 \\
1097 \\
1158 \\
1390 \\
1463 \\
1530 \\
1695\end{array}$ \\
\hline $\begin{array}{l}339 \\
340 \\
341 \\
342 \\
343 \\
344 \\
345 \\
346 \\
347 \\
348\end{array}$ & $\begin{array}{ll}34 & 19.78 \\
34 & 19.96 \\
34 & 29.90 \\
34 & 29.31 \\
34 & 28.72 \\
34 & 28.19 \\
34 & 27.66 \\
34 & 27.06 \\
34 & 26.49 \\
34 & 26.01\end{array}$ & $\begin{array}{ll}117 & 50.22 \\
117 & 50.26 \\
117 & 45.85 \\
117 & 45.85 \\
117 & 45.85 \\
117 & 45.85 \\
117 & 45.85 \\
117 & 45.84 \\
117 & 45.86 \\
117 & 46.06\end{array}$ & $\begin{array}{l}1890 \\
1932 \\
1042 \\
1082 \\
1131 \\
1180 \\
1231 \\
1286 \\
1341 \\
1402\end{array}$ \\
\hline
\end{tabular}


TABLE 2.-Seismic Recorder Locations, western Mojave Desert, 1980-1981-Continued

\begin{tabular}{|c|c|c|c|}
\hline $\begin{array}{l}\text { Station } \\
\text { number }\end{array}$ & $\begin{array}{l}\text { Latitude } \\
\text { deg min }\end{array}$ & $\begin{array}{l}\text { Longitude } \\
\text { deg min }\end{array}$ & $\begin{array}{c}\text { Elevation } \\
\text { meters }\end{array}$ \\
\hline $\begin{array}{l}349 \\
350 \\
351 \\
352 \\
353 \\
355 \\
356 \\
357 \\
358 \\
359\end{array}$ & $\begin{array}{ll}34 & 25.33 \\
34 & 24.74 \\
34 & 24.45 \\
34 & 24.88 \\
34 & 25.08 \\
34 & 24.08 \\
34 & 23.90 \\
34 & 23.73 \\
34 & 23.65 \\
34 & 23.53\end{array}$ & $\begin{array}{ll}117 & 46.02 \\
117 & 46.12 \\
117 & 45.83 \\
117 & 46.58 \\
117 & 47.38 \\
117 & 48.85 \\
117 & 48.57 \\
117 & 47.95 \\
117 & 47.58 \\
117 & 47.09\end{array}$ & $\begin{array}{l}1469 \\
1567 \\
1628 \\
1585 \\
1534 \\
1378 \\
1414 \\
1487 \\
1530 \\
1585\end{array}$ \\
\hline $\begin{array}{l}360 \\
361 \\
362 \\
363 \\
364 \\
365 \\
366 \\
367 \\
368 \\
369\end{array}$ & $\begin{array}{ll}34 & 23.50 \\
34 & 23.35 \\
34 & 23.17 \\
34 & 22.94 \\
34 & 22.85 \\
34 & 22.70 \\
34 & 22.52 \\
34 & 22.42 \\
34 & 22.45 \\
34 & 22.55\end{array}$ & $\begin{array}{ll}117 & 46.64 \\
117 & 46.52 \\
117 & 46.35 \\
117 & 46.19 \\
117 & 45.93 \\
117 & 45.70 \\
117 & 45.21 \\
117 & 45.07 \\
117 & 45.71 \\
117 & 46.19\end{array}$ & $\begin{array}{l}1634 \\
1646 \\
1689 \\
1743 \\
1792 \\
1859 \\
1975 \\
2010 \\
2060 \\
2109\end{array}$ \\
\hline $\begin{array}{l}370 \\
371 \\
372 \\
373 \\
374 \\
375 \\
376 \\
377 \\
378 \\
379\end{array}$ & $\begin{array}{ll}34 & 22.63 \\
34 & 22.25 \\
34 & 22.10 \\
34 & 21.66 \\
34 & 21.59 \\
34 & 22.03 \\
34 & 21.73 \\
34 & 21.35 \\
34 & 20.90 \\
34 & 20.89\end{array}$ & $\begin{array}{ll}117 & 46.71 \\
117 & 46.83 \\
117 & 47.18 \\
117 & 47.38 \\
117 & 47.85 \\
117 & 47.96 \\
117 & 48.50 \\
117 & 48.49 \\
117 & 48.63 \\
117 & 49.13\end{array}$ & $\begin{array}{l}2170 \\
2219 \\
2256 \\
2310 \\
2353 \\
2402 \\
2377 \\
2329 \\
2286 \\
2231\end{array}$ \\
\hline $\begin{array}{l}380 \\
381 \\
382 \\
383 \\
384 \\
385 \\
386 \\
387 \\
388 \\
389\end{array}$ & $\begin{array}{rr}34 & 20.74 \\
34 & 9.75 \\
34 & 9.80 \\
34 & 10.22 \\
34 & 10.86 \\
34 & 11.10 \\
34 & 11.31 \\
34 & 11.59 \\
34 & 11.82 \\
34 & 12.03\end{array}$ & $\begin{array}{ll}117 & 49.57 \\
117 & 53.76 \\
117 & 53.19 \\
117 & 53.23 \\
117 & 52.82 \\
117 & 52.26 \\
117 & 52.07 \\
117 & 51.86 \\
117 & 51.71 \\
117 & 51.46\end{array}$ & $\begin{array}{r}2213 \\
250 \\
280 \\
329 \\
390 \\
396 \\
387 \\
364 \\
384 \\
427\end{array}$ \\
\hline
\end{tabular}


TABLE 2.-Seismic Recorder Locations, western Mojave Desert, 1980.1981-Continued

\begin{tabular}{|c|c|c|c|}
\hline $\begin{array}{l}\text { Station } \\
\text { number }\end{array}$ & $\begin{array}{l}\text { Latitude } \\
\text { deg min }\end{array}$ & $\begin{array}{l}\text { Longitude } \\
\text { deg min }\end{array}$ & $\begin{array}{c}\text { Elevation } \\
\text { meters }\end{array}$ \\
\hline $\begin{array}{l}390 \\
391 \\
392 \\
393 \\
394 \\
395 \\
396 \\
397 \\
398 \\
399\end{array}$ & $\begin{array}{ll}34 & 12.23 \\
34 & 12.40 \\
34 & 12.63 \\
34 & 12.86 \\
34 & 13.01 \\
34 & 13.37 \\
34 & 13.60 \\
34 & 13.76 \\
34 & 13.95 \\
34 & 14.10\end{array}$ & $\begin{array}{ll}117 & 51.87 \\
117 & 51.89 \\
117 & 51.84 \\
117 & 51.70 \\
117 & 51.29 \\
117 & 51.03 \\
117 & 50.82 \\
117 & 50.69 \\
117 & 50.71 \\
117 & 50.82\end{array}$ & $\begin{array}{l}463 \\
488 \\
502 \\
506 \\
506 \\
469 \\
469 \\
469 \\
469 \\
469\end{array}$ \\
\hline $\begin{array}{l}400 \\
401 \\
441 \\
442 \\
443 \\
444 \\
445 \\
446 \\
447 \\
448\end{array}$ & $\begin{array}{ll}34 & 14.26 \\
34 & 44.30 \\
35 & 42.40 \\
35 & 41.26 \\
35 & 40.66 \\
35 & 40.08 \\
35 & 38.52 \\
35 & 37.66 \\
35 & 36.63 \\
35 & 35.91\end{array}$ & $\begin{array}{ll}117 & 51.03 \\
117 & 46.35 \\
117 & 31.88 \\
117 & 31.93 \\
117 & 31.92 \\
117 & 31.93 \\
117 & 31.53 \\
117 & 31.21 \\
117 & 31.46 \\
117 & 31.97\end{array}$ & $\begin{array}{l}457 \\
933 \\
750 \\
660 \\
622 \\
600 \\
634 \\
683 \\
759 \\
863\end{array}$ \\
\hline $\begin{array}{l}449 \\
450 \\
451 \\
452 \\
453 \\
454 \\
455 \\
456 \\
457 \\
458\end{array}$ & $\begin{array}{ll}35 & 35.17 \\
35 & 34.36 \\
35 & 33.91 \\
35 & 32.92 \\
35 & 32.05 \\
35 & 31.27 \\
35 & 30.62 \\
35 & 30.02 \\
35 & 29.25 \\
35 & 28.74\end{array}$ & $\begin{array}{ll}117 & 32.48 \\
117 & 33.14 \\
117 & 33.69 \\
117 & 34.59 \\
117 & 34.87 \\
117 & 34.97 \\
117 & 35.08 \\
117 & 35.18 \\
117 & 35.29 \\
117 & 35.37\end{array}$ & $\begin{array}{r}957 \\
1000 \\
986 \\
981 \\
896 \\
861 \\
863 \\
884 \\
945 \\
1003\end{array}$ \\
\hline $\begin{array}{l}459 \\
460 \\
461 \\
462 \\
463 \\
464 \\
465 \\
466 \\
467 \\
468\end{array}$ & $\begin{array}{lr}35 & 28.21 \\
35 & 41.78 \\
34 & 55.29 \\
34 & 55.75 \\
34 & 56.38 \\
34 & 57.22 \\
34 & 58.36 \\
34 & 58.92 \\
34 & 59.29 \\
35 & 0.05\end{array}$ & $\begin{array}{ll}117 & 35.41 \\
117 & 31.89 \\
117 & 45.58 \\
117 & 44.69 \\
117 & 44.26 \\
117 & 43.97 \\
117 & 44.03 \\
117 & 43.00 \\
117 & 42.26 \\
117 & 41.07\end{array}$ & $\begin{array}{r}1033 \\
683 \\
719 \\
732 \\
723 \\
713 \\
707 \\
724 \\
732 \\
739\end{array}$ \\
\hline
\end{tabular}


TABLE 2.-Seismic Recorder Locations, western Mojave Desert, 1980-1981-Continued

\begin{tabular}{|c|c|c|c|}
\hline $\begin{array}{l}\text { Station } \\
\text { number }\end{array}$ & $\begin{array}{l}\text { Latitude } \\
\text { deg min }\end{array}$ & $\begin{array}{l}\text { Longitude } \\
\text { deg min }\end{array}$ & $\begin{array}{c}\text { Elevation } \\
\text { meters }\end{array}$ \\
\hline $\begin{array}{l}469 \\
470 \\
471 \\
472 \\
473 \\
474 \\
475 \\
476 \\
477 \\
478\end{array}$ & $\begin{array}{ll}35 & 0.99 \\
35 & 1.25 \\
35 & 1.70 \\
35 & 1.98 \\
35 & 2.64 \\
35 & 3.09 \\
35 & 4.01 \\
35 & 4.82 \\
35 & 5.84 \\
35 & 7.07\end{array}$ & $\begin{array}{ll}117 & 40.56 \\
117 & 40.55 \\
117 & 40.55 \\
117 & 40.82 \\
117 & 40.67 \\
117 & 40.30 \\
117 & 40.89 \\
117 & 40.93 \\
117 & 41.05 \\
117 & 40.82\end{array}$ & $\begin{array}{l}742 \\
753 \\
744 \\
750 \\
770 \\
771 \\
770 \\
780 \\
796 \\
811\end{array}$ \\
\hline $\begin{array}{l}479 \\
480 \\
481 \\
482 \\
483 \\
484 \\
485 \\
486 \\
487 \\
488\end{array}$ & $\begin{array}{rr}35 & 8.08 \\
35 & 8.74 \\
35 & 27.74 \\
35 & 27.20 \\
35 & 26.65 \\
35 & 26.01 \\
35 & 25.21 \\
35 & 24.06 \\
35 & 23.11 \\
35 & 22.29\end{array}$ & $\begin{array}{ll}117 & 40.11 \\
117 & 39.81 \\
117 & 35.27 \\
117 & 35.08 \\
117 & 35.37 \\
117 & 35.43 \\
117 & 35.51 \\
117 & 35.75 \\
117 & 35.99 \\
117 & 36.40\end{array}$ & $\begin{array}{r}832 \\
814 \\
1049 \\
1049 \\
1009 \\
991 \\
963 \\
960 \\
1012 \\
1085\end{array}$ \\
\hline $\begin{array}{l}489 \\
490 \\
491 \\
492 \\
493 \\
494 \\
495 \\
496 \\
497 \\
498\end{array}$ & $\begin{array}{ll}35 & 21.84 \\
35 & 20.67 \\
35 & 19.59 \\
35 & 18.62 \\
35 & 17.62 \\
35 & 16.42 \\
35 & 15.52 \\
35 & 14.30 \\
35 & 13.35 \\
35 & 11.96\end{array}$ & $\begin{array}{ll}117 & 38.06 \\
117 & 37.82 \\
117 & 37.83 \\
117 & 38.29 \\
117 & 38.76 \\
117 & 39.47 \\
117 & 39.48 \\
117 & 39.46 \\
117 & 39.45 \\
117 & 39.95\end{array}$ & $\begin{array}{r}1158 \\
1116 \\
1074 \\
1026 \\
988 \\
957 \\
927 \\
899 \\
871 \\
846\end{array}$ \\
\hline $\begin{array}{l}499 \\
500 \\
510 \\
577 \\
578 \\
580 \\
583\end{array}$ & $\begin{array}{rr}35 & 10.69 \\
35 & 9.83 \\
34 & 35.95 \\
35 & 6.44 \\
35 & 7.44 \\
35 & 8.53 \\
35 & 26.79\end{array}$ & $\begin{array}{ll}117 & 39.98 \\
117 & 39.52 \\
117 & 48.51 \\
117 & 40.86 \\
117 & 40.65 \\
117 & 40.00 \\
117 & 35.26\end{array}$ & $\begin{array}{r}832 \\
823 \\
826 \\
798 \\
819 \\
812 \\
1018\end{array}$ \\
\hline
\end{tabular}


TABLE 3.-Explanantion of Columns Seismic Recorder Data Table

\begin{tabular}{|c|c|}
\hline Column & Description \\
\hline $\begin{array}{r}\text { Loc } \\
\text { Dist } \\
\text { Azim } \\
\text { Db }\end{array}$ & $\begin{array}{l}\text { Location number of seismic recorder. } \\
\text { Distance from shotpoint to recorder. } \\
\text { Azimuth from shotpoint to recorder, in degrees, clockwise from north. } \\
\text { Amplifier attenuation setting }(\mathrm{db}) \text { of the digitized channel. } \\
\text { The gain is determined by subtracting the given attenuation from } \\
\text { a total gain of } 96 \mathrm{db} \text {. }\end{array}$ \\
\hline
\end{tabular}

TABLE 4.-Description of Tape Grade Codes

\begin{tabular}{clcl}
\hline Code & Description & Code & Description \\
\hline 0 & Good playback & 14 & Tape Speed off \\
1 & Tape did not run & 15 & Bad Calibration \\
2 & No signal & 16 & Recorder stopped during play \\
3 & Skipped record time & 17 & Bad time code \\
6 & Weak multiplexed signal; & 18 & Tape speed off, belt slipped \\
& cannot read time code; & 20 & Recorder unit not deployed \\
& low record level & 25 & Bad geophone test \\
7 & Continuous calibration & 28 & Unit damaged \\
8 & Noise, sinusoidal & 29 & Tape damaged \\
9 & Noise, spike & 30 & Digitized without calibraiton \\
10 & Noise, WWVB cross feed & 31 & Amplifier out of balance \\
12 & Noise, random & & \\
\hline
\end{tabular}


TABLE 5.-Seismic Recorder Data

Shot Number 1 Shot Point 4

Shot Time (Julian day, hr, min, sec): 167:06:00:00.010

\begin{tabular}{|c|c|c|c|c|c|c|c|c|c|c|c|c|c|c|}
\hline Loc & $\begin{array}{l}\text { Dist } \\
(\mathbf{k m})\end{array}$ & $\begin{array}{l}\text { Azim } \\
(\text { deg })\end{array}$ & $\mathbf{D b}$ & $\begin{array}{l}\text { Tape } \\
\text { Grade }\end{array}$ & Loc & $\begin{array}{c}\text { Dist } \\
(\mathbf{k m})\end{array}$ & $\begin{array}{l}\text { Azim } \\
\text { (deg) }\end{array}$ & $\mathrm{Db}$ & $\begin{array}{l}\text { Tape } \\
\text { Grede }\end{array}$ & Loc & $\begin{array}{c}\text { Dist } \\
(\mathbf{k m})\end{array}$ & $\begin{array}{l}\text { Azim } \\
\text { (deg) }\end{array}$ & $\mathrm{Db}$ & $\begin{array}{l}\text { Tape } \\
\text { Grade }\end{array}$ \\
\hline 101 & 16.34 & 322.5 & 26 & 0 & 135 & 4.93 & 281.3 & 44 & 15 & 169 & 18.61 & 261.2 & 44 & 0 \\
\hline 102 & 15.78 & 319.1 & 26 & 0 & 136 & 5.42 & 279.3 & 44 & 0 & 170 & 18.10 & 261.0 & 44 & 0 \\
\hline 103 & 14.93 & 319.0 & & $6 / 17$ & 137 & 5.93 & 277.5 & 44 & 15 & 171 & 17.52 & 260.7 & & $0 / 6$ \\
\hline 104 & 14.14 & 318.2 & 26 & 0 & 138 & 6.41 & 276.0 & 44 & 0 & 172 & 16.98 & 260.4 & 44 & 0 \\
\hline 105 & 13.60 & 315.5 & 44 & 0 & 139 & 6.98 & 274.4 & 44 & 13 & 173 & 16.49 & 260.1 & & $6 / 17$ \\
\hline 106 & 12.72 & 313.2 & & 2 & 141 & 17.24 & 221.1 & 26 & 0 & 174 & 15.93 & 259.7 & 44 & 0 \\
\hline 107 & 12.38 & 311.4 & 44 & 0 & 142 & 16.63 & 220.7 & 26 & 0 & 175 & 15.45 & 259.4 & 44 & $\mathbf{0}$ \\
\hline 108 & 12.12 & 309.9 & 44 & 0 & 143 & 16.20 & 222.0 & 44 & $\mathbf{0}$ & 176 & 14.90 & 259.0 & 44 & 0 \\
\hline 109 & 12.33 & 307.0 & 44 & 0 & 144 & 15.81 & 223.3 & 44 & 0 & 177 & 14.36 & 258.6 & 44 & 0 \\
\hline 110 & 12.49 & 305.0 & 44 & 0 & 145 & 15.43 & 224.6 & 44 & 15 & 178 & 13.82 & 258.2 & 44 & 0 \\
\hline 111 & 12.72 & 302.6 & & 2 & 146 & 15.02 & 226.2 & 44 & 0 & 179 & 13.31 & 257.7 & 44 & $\mathbf{0}$ \\
\hline 112 & 12.40 & 300.5 & 44 & 0 & 147 & 14.66 & 227.6 & 44 & $\mathbf{0}$ & 180 & 12.74 & 257.2 & 44 & 0 \\
\hline 113 & 12.16 & 298.3 & & 25 & 148 & 14.29 & 229.3 & 44 & 0 & 181 & 11.98 & 252.2 & 44 & 0 \\
\hline 114 & 11.85 & 295.2 & 44 & 15 & 149 & 13.94 & 231.0 & 44 & 0 & 182 & 11.07 & 258.3 & 44 & 0 \\
\hline 115 & 11.72 & 293.6 & 44 & 0 & 150 & 13.61 & 232.7 & 44 & 0 & 183 & 10.97 & 261.0 & 44 & 0 \\
\hline 116 & 11.51 & 291.2 & 44 & $0 / 6 / 30$ & 151 & 13.29 & 234.6 & 44 & 0 & 184 & 10.90 & 263.8 & 44 & 0 \\
\hline 117 & 11.36 & 289.0 & 44 & 0 & 152 & 12.98 & 236.6 & 44 & 0 & 185 & 11.70 & 256.1 & & 1 \\
\hline 118 & 11.19 & 286.3 & 44 & 0 & 153 & 12.70 & 238.6 & 26 & 0 & 186 & 12.21 & 256.7 & 44 & 15 \\
\hline 119 & 11.05 & 283.9 & 26 & 0 & 154 & 12.42 & 240.8 & 26 & 25 & 187 & 7.42 & 273.4 & 44 & $\mathbf{0}$ \\
\hline 121 & 0.02 & 29.9 & 62 & 0 & 155 & 12.18 & 242.9 & 44 & 0 & 188 & 7.94 & 272.2 & 44 & $\mathbf{0}$ \\
\hline 122 & 2.36 & 98.7 & 62 & 0 & 156 & 11.94 & 245.2 & 44 & 0 & 189 & 8.46 & 271.4 & & 3 \\
\hline 123 & 1.93 & 98.4 & 62 & 0 & 157 & 11.73 & 247.7 & 44 & 15 & 190 & 8.97 & 270.6 & 44 & 0 \\
\hline 124 & 1.39 & 98.0 & 62 & 0 & 158 & 11.52 & 250.3 & & 1 & 191 & 9.49 & 269.8 & 44 & 0 \\
\hline 125 & 0.86 & 96.7 & 62 & 0 & 159 & 11.35 & 252.8 & 44 & 0 & 192 & 10.01 & 269.3 & 44 & 0 \\
\hline 126 & 0.21 & 292.4 & 62 & 0 & 160 & 11.21 & 255.4 & 44 & $\mathbf{0}$ & 193 & 10.54 & 268.8 & 44 & 0 \\
\hline 127 & 0.73 & 283.9 & 62 & 0 & 161 & 21.29 & 257.7 & 44 & 0 & 194 & 10.82 & 268.5 & & 1 \\
\hline 128 & 1.28 & 282.1 & 62 & 0 & 162 & 22.37 & 262.7 & 44 & 0 & 195 & 10.84 & 265.7 & 44 & 0 \\
\hline 129 & 1.81 & 281.3 & 62 & 0 & 163 & 21.83 & 262.5 & 44 & $\mathbf{0}$ & 196 & 10.84 & 271.3 & & 1 \\
\hline 130 & 2.32 & 281.8 & 62 & 0 & 164 & 21.29 & 262.3 & 26 & 0 & 197 & 10.85 & 272.5 & 44 & 0 \\
\hline 131 & 2.84 & 278.5 & 62 & 0 & 165 & 20.78 & 262.1 & 26 & 0 & 198 & 10.88 & 275.3 & 44 & 0 \\
\hline 132 & 3.36 & 279.5 & 62 & 0 & 166 & 20.23 & 261.9 & 26 & 0 & 199 & 10.95 & 278.0 & & 1 \\
\hline 133 & 3.92 & 280.4 & 62 & $\mathbf{0}$ & 167 & 19.69 & 261.7 & 26 & 0 & 200 & 11.05 & 280.9 & & 1 \\
\hline 134 & 4.45 & 280.5 & & 3 & 168 & 19.18 & 261.5 & 44 & 0 & & & & & \\
\hline
\end{tabular}


TABLE 5.-Seismic Recorder Data-Continued Shot Number 2 Shot Point 2

Shot Time (Julian day, hr, min, sec): 167:06:29:60.003

\begin{tabular}{|c|c|c|c|c|c|c|c|c|c|c|c|c|c|c|}
\hline Loc & $\begin{array}{l}\text { Dist } \\
(\mathbf{k m})\end{array}$ & $\begin{array}{l}\text { Azim } \\
\text { (deg) }\end{array}$ & Db & $\begin{array}{l}\text { Tape } \\
\text { Grade }\end{array}$ & Loc & $\begin{array}{c}\text { Dist } \\
(\mathbf{k m})\end{array}$ & $\begin{array}{l}\text { Arim } \\
\text { (deg) }\end{array}$ & $\mathbf{D b}$ & $\begin{array}{l}\text { Tape } \\
\text { Grade }\end{array}$ & Loc & $\begin{array}{c}\text { Dist } \\
(\mathrm{km})\end{array}$ & $\begin{array}{l}\text { Azim } \\
\text { (deg) }\end{array}$ & $\mathbf{D b}$ & $\begin{array}{l}\text { Tape } \\
\text { Grade }\end{array}$ \\
\hline 101 & 16.67 & 5.0 & 26 & 0 & 135 & 8.02 & 54.8 & 26 & 15 & 169 & 7.04 & 276.5 & 44 & 0 \\
\hline 102 & 15.61 & 3.9 & 26 & 15 & 136 & 7.56 & 53.2 & 26 & 0 & 170 & 6.53 & 277.0 & 44 & 0 \\
\hline 103 & 14.99 & 6.1 & & $6 / 17$ & 137 & 7.07 & 51.3 & 44 & 15 & 171 & 5.94 & 277.8 & & 17 \\
\hline 104 & 14.32 & 7.9 & 26 & 0 & 138 & 6.62 & 49.3 & 44 & 0 & 172 & 5.40 & 278.7 & 44 & 0 \\
\hline 105 & 13.48 & 7.9 & 26 & 0 & 139 & 6.10 & 46.7 & 44 & 0 & 173 & 4.91 & 279.6 & 44 & $0 / 30$ \\
\hline 106 & 12.54 & 9.7 & 26 & 0 & 141 & 9.36 & 179.5 & 44 & 0 & 174 & 4.34 & 280.5 & 44 & 0 \\
\hline 107 & 12.02 & 10.1 & 26 & 0 & 142 & 8.99 & 176.3 & 44 & 0 & 175 & 3.87 & 281.9 & 44 & 0 \\
\hline 108 & 11.62 & 10.4 & 26 & 0 & 143 & 8.42 & 176.1 & 44 & 0 & 176 & 3.32 & 283.9 & & 2 \\
\hline 109 & 11.17 & $7 . \theta$ & 26 & 0 & 144 & 7.89 & 175.8 & 44 & 0 & 177 & 2.79 & 286.8 & 62 & 0 \\
\hline 110 & 10.88 & 6.1 & 26 & 0 & 145 & 7.36 & 175.5 & 44 & 15 & 178 & 2.27 & 290.7 & 62 & 0 \\
\hline 111 & 10.51 & 3.7 & & 2 & 146 & 6.79 & 175.1 & 44 & 0 & 179 & 1.80 & $296 . \theta$ & 62 & 0 \\
\hline 112 & 9.95 & 4.1 & 26 & 0 & 147 & 6.26 & 174.7 & 44 & 0 & 180 & 1.31 & 308.7 & 62 & 0 \\
\hline 113 & 9.43 & 4.1 & & 7 & 148 & 5.71 & 174.1 & 44 & 0 & 181 & 0.02 & 180.0 & 62 & 0 \\
\hline 114 & 8.73 & 4.4 & 26 & 15 & 149 & 5.16 & 173.6 & 44 & 0 & 182 & 1.50 & 22.0 & 62 & 0 \\
\hline 115 & 8.35 & 4.5 & 26 & 0 & 160 & 4.64 & 172.9 & 62 & 0 & 183 & 2.01 & 16.4 & 62 & 0 \\
\hline 116 & 7.84 & 4.9 & 44 & 15 & 151 & 4.09 & 171.9 & 62 & 0 & 184 & 2.52 & 13.0 & 62 & 0 \\
\hline 117 & 7.38 & 5.1 & 44 & 0 & 152 & 3.55 & 170.7 & 44 & 0 & 185 & 0.83 & 3.3 & & 1 \\
\hline 118 & 6.83 & 5.5 & 44 & 0 & 153 & 3.03 & 169.2 & 44 & 0 & 186 & 0.95 & 330.1 & 62 & 0 \\
\hline 119 & 6.34 & 6.1 & 44 & 0 & 154 & 2.48 & 166.9 & & 25 & 187 & 5.71 & 44.3 & 44 & 0 \\
\hline 121 & 11.99 & 72.1 & 26 & 0 & 155 & 1.98 & 163.5 & 62 & 0 & 188 & 5.25 & 41.3 & 44 & 0 \\
\hline 122 & 14.12 & 76.5 & 26 & 0 & 156 & 1.47 & 157.5 & 62 & 0 & 189 & 4.85 & 37.4 & & 2 \\
\hline 123 & 13.73 & 75.8 & 26 & 0 & 157 & 0.98 & 145.7 & 62 & 0 & 190 & 4.46 & 33.1 & 44 & 0 \\
\hline 124 & 13.24 & 74.8 & 26 & 0 & 158 & 0.61 & 113.5 & & 1 & 181 & 4.09 & 27.9 & 44 & 0 \\
\hline 125 & 12.76 & 73.8 & 26 & 0 & 159 & 0.63 & 62.2 & 62 & 0 & 182 & 3.79 & 21.5 & 44 & 0 \\
\hline 126 & 11.81 & 71.6 & 26 & 0 & 160 & $0 . \theta \theta$ & 34.0 & 62 & 0 & 183 & 3.52 & 14.1 & 62 & 0 \\
\hline 127 & 11.35 & 70.3 & 26 & 0 & 161 & 9.44 & 264.5 & 44 & 0 & 194 & 3.41 & 9.9 & & 1 \\
\hline 128 & 10.88 & 68.9 & 26 & 0 & 162 & 10.81 & 274.1 & 44 & 0 & 195 & 2.89 & 11.7 & 62 & 0 \\
\hline 129 & 10.42 & 67.4 & 44 & 0 & 163 & 10.27 & 274.3 & 44 & 0 & 196 & 3.93 & 8.2 & & 1 \\
\hline 130 & 10.02 & 65.6 & 26 & 0 & 164 & 9.73 & 274.6 & 44 & $0 / 30$ & 197 & 4.16 & 7.7 & 62 & 0 \\
\hline 131 & $\mathbf{9 . 5 1}$ & 64.6 & 44 & 0 & 165 & 9.21 & 274.8 & 44 & 0 & 198 & 4.68 & 6.8 & 44 & 0 \\
\hline 132 & $\theta .11$ & 62.5 & 44 & 0 & 166 & 8.66 & 275.1 & 44 & 0 & $18 \theta$ & 5.21 & 6.1 & & 1 \\
\hline 133 & 8.72 & 60.0 & 26 & 0 & 167 & 8.12 & 275.5 & 44 & 0 & 200 & 5.76 & 5.5 & & 1 \\
\hline 134 & 8.33 & 57.6 & & 3 & 168 & 7.61 & 275.9 & & 7 & & & & & \\
\hline
\end{tabular}


TABLE 5.-Seismic Recorder Data-Continued Shot Number 3 Shot Point 3

Shot Time (Julian day, hr, min, sec): 167:07:00:00.008

\begin{tabular}{|c|c|c|c|c|c|c|c|c|c|c|c|c|c|c|}
\hline Loc & $\begin{array}{c}\text { Dist } \\
\text { (kin) }\end{array}$ & $\begin{array}{l}\text { Azim } \\
\text { (deg) }\end{array}$ & $\mathbf{D b}$ & $\begin{array}{l}\text { Tepe } \\
\text { Grede }\end{array}$ & Loc & $\begin{array}{c}\text { Dist } \\
(\mathbf{k m})\end{array}$ & $\begin{array}{l}\text { Azim } \\
\text { (deg) }\end{array}$ & $\mathbf{D b}$ & $\begin{array}{l}\text { Tape } \\
\text { Grade }\end{array}$ & Loc & $\begin{array}{c}\text { Dist } \\
(\mathbf{k m})\end{array}$ & $\begin{array}{l}\text { Azim } \\
\text { (deg) }\end{array}$ & $\mathbf{D b}$ & $\begin{array}{r}\text { Tape } \\
\text { Grade }\end{array}$ \\
\hline 101 & 0.08 & 138.8 & 62 & 0 & 135 & 13.11 & 156.8 & 26 & 0 & 169 & 17.96 & 207.8 & 26 & 0 \\
\hline 102 & 1.14 & 197.2 & 62 & 0 & 136 & 13.01 & 159.0 & 26 & 0 & 170 & 17.72 & 206.4 & 26 & 0 \\
\hline 103 & 1.78 & 173.8 & & $6 / 17$ & 137 & 12.93 & 161.4 & 26 & 0 & 171 & 17.46 & 204.7 & & $6 / 17$ \\
\hline 104 & 2.55 & 167.1 & 44 & 0 & 138 & 12.88 & 163.6 & 44 & 0 & 172 & 17.23 & 203.0 & 26 & 0 \\
\hline 105 & 3.35 & 172.0 & 44 & 0 & 139 & 12.86 & 166.3 & 26 & 0 & 173 & 17.04 & 201.5 & 26 & 0 \\
\hline 106 & 4.38 & 170.5 & 44 & 0 & 141 & 26.06 & 182.9 & 26 & 0 & 174 & 16.86 & 199.6 & 26 & 0 \\
\hline 107 & 4.89 & 171.7 & 44 & 0 & 142 & 25.66 & 181.8 & & 25 & 175 & 16.70 & 198.1 & 26 & 0 \\
\hline 108 & 5.29 & 172.4 & 44 & 0 & 143 & 25.08 & 181.9 & 26 & 0 & 176 & 16.53 & 196.2 & & $6 / 17$ \\
\hline 109 & 5.61 & 178.5 & 44 & 0 & 144 & 24.55 & 181.9 & 26 & 0 & 177 & 16.38 & 194.4 & 26 & 0 \\
\hline 110 & 5.86 & 182.2 & 44 & 0 & 145 & 24.02 & 182.0 & 26 & $0 / 15$ & 178 & 16.25 & 192.5 & 26 & 25 \\
\hline 111 & 6.22 & 186.7 & & 17 & 146 & 23.45 & 182.0 & 26 & 0 & 179 & 16.14 & 190.7 & 26 & 0 \\
\hline 112 & 6.78 & 185.8 & 44 & 0 & 147 & 22.92 & 182.0 & 26 & 0 & 180 & 16.03 & 188.7 & 26 & 0 \\
\hline 113 & 7.30 & 185.6 & & 25 & 148 & 22.37 & 182.1 & 26 & 0 & 181 & 16.75 & 184.8 & 26 & 0 \\
\hline 114 & 8.00 & 185.2 & 44 & 0 & 149 & 21.82 & 182.2 & 26 & 0 & 182 & 15.30 & 183.1 & 26 & 0 \\
\hline 115 & 8.38 & 185.1 & 44 & 0 & 150 & 21.29 & 182.2 & 26 & 0 & 183 & 14.77 & 183.2 & 26 & 0 \\
\hline 116 & 8.89 & 184.7 & 26 & 0 & 151 & 20.74 & 182.3 & 26 & 0 & 184 & 14.24 & 183.3 & & 25 \\
\hline 117 & 9.35 & 184.5 & 26 & 0 & 152 & 20.19 & 182.3 & 26 & 0 & 185 & 15.90 & 184.9 & & 1 \\
\hline 118 & 9.90 & 184.3 & 26 & 0 & 153 & 19.66 & 182.4 & 26 & 0 & 186 & 15.96 & 186.7 & 26 & 0 \\
\hline 119 & 10.40 & 184.0 & 26 & 0 & 154 & 19.11 & 182.5 & & 25 & 187 & 12.85 & 168.3 & & 3 \\
\hline 121 & 16.41 & 142.4 & 26 & 0 & 155 & 18.59 & 182.6 & 26 & 0 & 188 & 12.89 & 170.8 & 26 & 0 \\
\hline 122 & 18.19 & 137.3 & 26 & 0 & 156 & 18.05 & 182.6 & 26 & 0 & 189 & 12.92 & 173.1 & & 3 \\
\hline 123 & 17.85 & 138.1 & 26 & 0 & 157 & 17.50 & 182.8 & 26 & 0 & 190 & 12.98 & 175.4 & 26 & 0 \\
\hline 124 & 17.44 & 139.2 & 26 & 0 & 158 & 16.93 & 182.8 & & 1 & 191 & 13.07 & 177.7 & 26 & 0 \\
\hline 125 & 17.03 & 140.4 & 26 & 0 & 159 & 16.40 & 182.9 & 26 & 0 & 192 & 13.14 & 180.0 & 26 & 0 \\
\hline 126 & 16.24 & 142.8 & 26 & 0 & 160 & 15.87 & 183.0 & 26 & 0 & 193 & 13.26 & 182.3 & 26 & 0 \\
\hline 127 & 15.85 & 144.1 & 26 & 0 & 161 & 20.62 & 211.5 & 26 & 0 & 194 & 13.34 & 183.5 & & 1 \\
\hline 128 & 15.47 & 145.5 & 26 & 0 & 162 & 20.02 & 217.5 & 26 & 0 & 195 & 13.87 & 183.3 & 26 & 0 \\
\hline 129 & 15.10 & 147.0 & 26 & 0 & 163 & 19.70 & 216.2 & 26 & 0 & 196 & 12.81 & 183.7 & & 1 \\
\hline 130 & 14.73 & 148.4 & 26 & 0 & 164 & 19.38 & 214.9 & 26 & 0 & 197 & 12.58 & 183.8 & 26 & 0 \\
\hline 131 & 14.51 & 150.3 & 26 & 0 & 165 & 19.09 & 213.6 & 26 & 0 & 198 & 12.05 & 184.0 & 26 & 0 \\
\hline 132 & 14.15 & 151.8 & 26 & 0 & 166 & 18.79 & 212.2 & 26 & 13 & 199 & 11.53 & 184.2 & & 1 \\
\hline 133 & 13.76 & 153.5 & 26 & 0 & 167 & 18.51 & 210.8 & 26 & 0 & 200 & 10.97 & 184.4 & & 1 \\
\hline 134 & 13.44 & 155.2 & & 3 & 168 & 18.24 & 209.4 & 26 & 0 & & & & & \\
\hline
\end{tabular}


TABLE 5.-Seismic Recorder Data-Continued

Shot Number \& Shot Point 1

Shot Time (Julian day, $\mathrm{hr}, \mathrm{min}, \mathrm{sec}$ ): 167:07:29:59.989

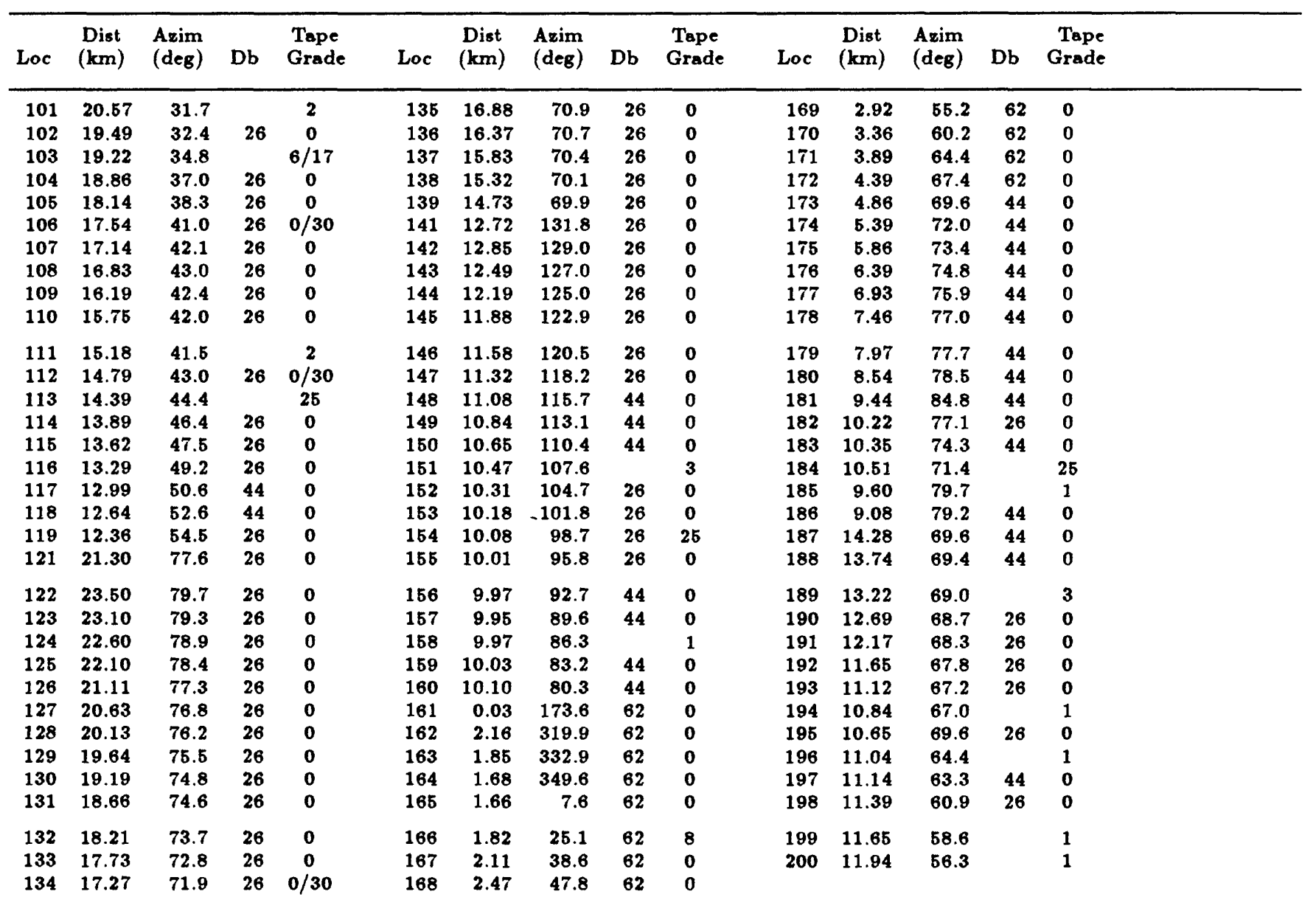


TABLE 5.-Seismic Recorder Data-Continued

Shot Number 5 Shot Point 5

Shot Time (Julian day, hr, min, sec): 167:08:30:00.010

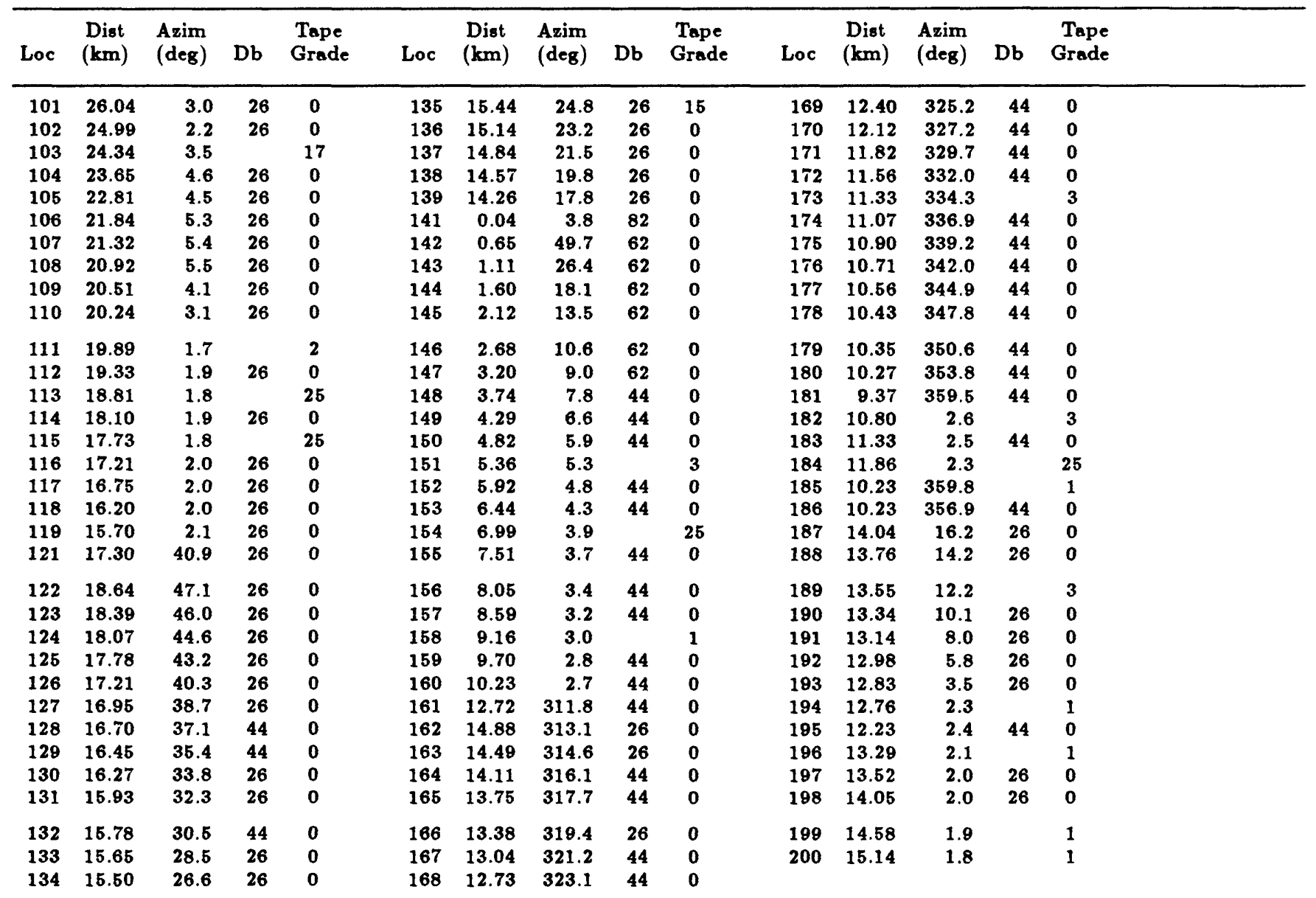


TABLE 5.-Seismic Recorder Data-Continued Shot Number 6 Shot Point 2

Shot Time (Julian day, hr, min, sec): 177:06:00:00.012

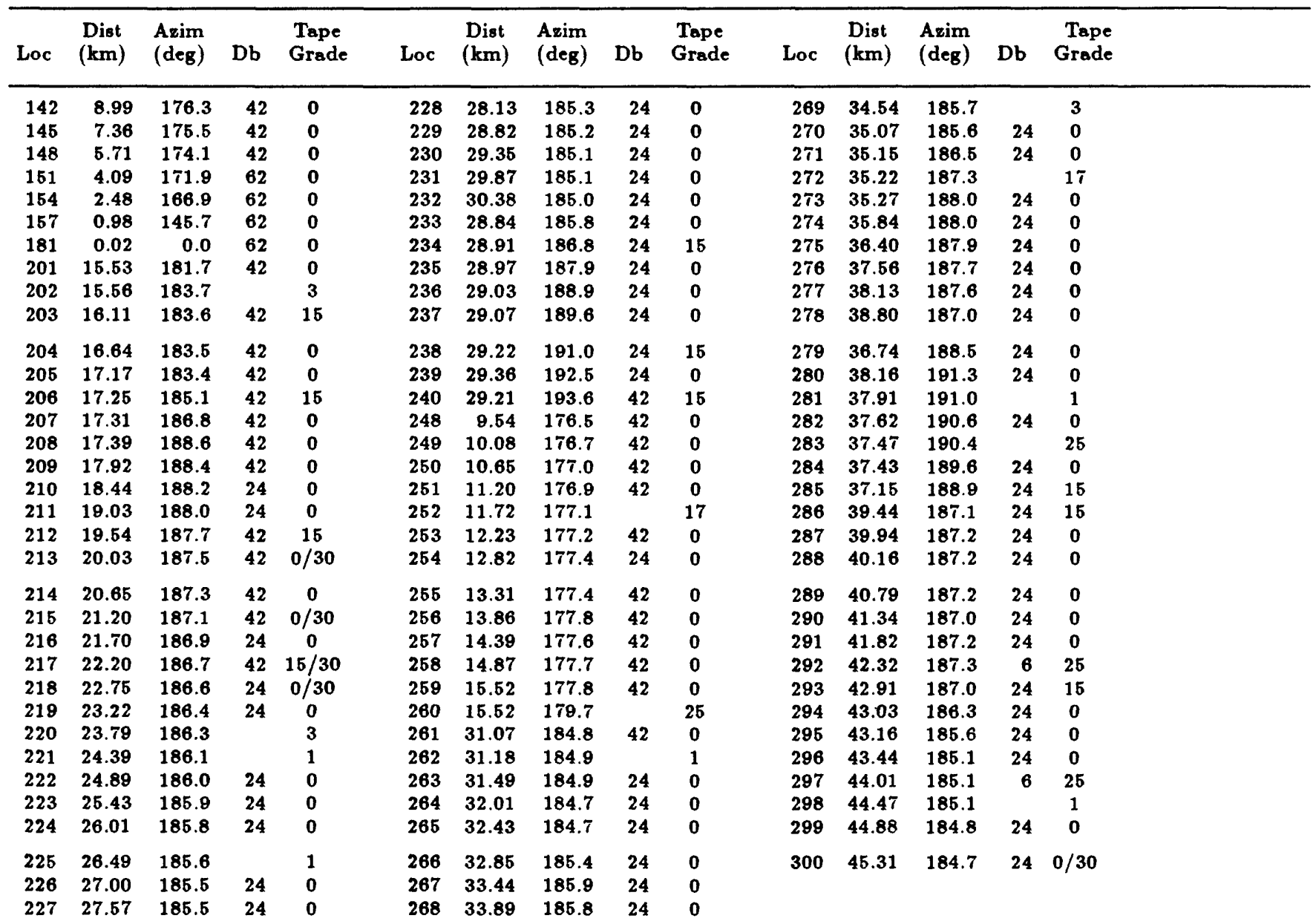


TABLE 5.-Seismic Recorder Data-Continued

Shot Number 7 Shot Point 7

Shot Time (Julian day, hr, min, sec): 177:07:00:00.012

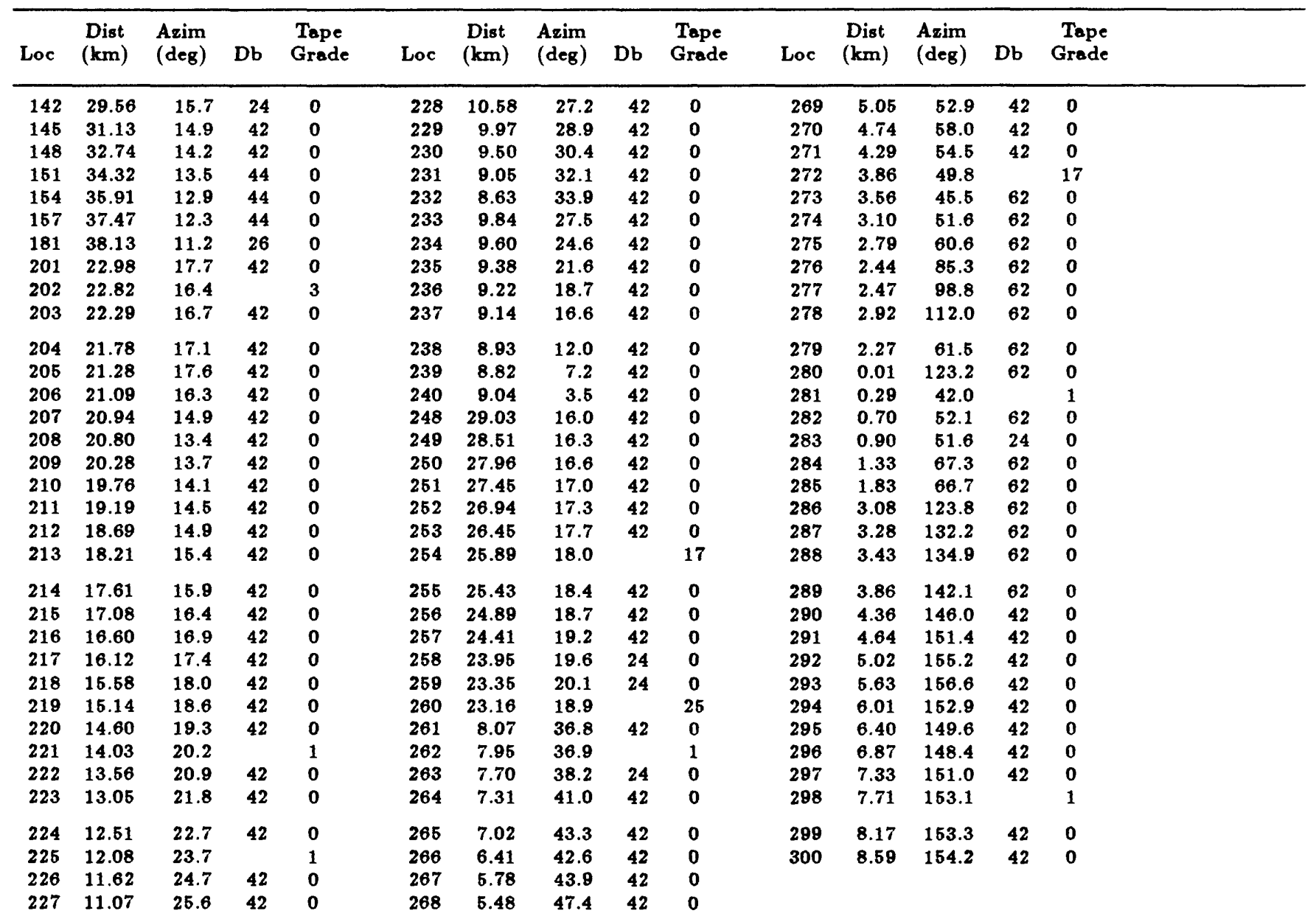


TABLE 5.-Seismic Recorder Data-Continued Shot Number 8 Shot Point 6

Shot Time (Julian day, hr, min, sec): 177:08:00:00.016

\begin{tabular}{|c|c|c|c|c|c|c|c|c|c|c|c|c|c|c|}
\hline Loc & $\begin{array}{c}\text { Dist } \\
(\mathbf{k m})\end{array}$ & $\begin{array}{l}\text { Axim } \\
\text { (deg) }\end{array}$ & $\mathrm{Db}$ & $\begin{array}{l}\text { Tape } \\
\text { Grade }\end{array}$ & Loc & $\begin{array}{c}\text { Dist } \\
(\mathbf{k m})\end{array}$ & $\begin{array}{l}\text { Azim } \\
(\text { deg })\end{array}$ & $\mathrm{Db}$ & $\begin{array}{l}\text { Tape } \\
\text { Grade }\end{array}$ & Loc & $\begin{array}{l}\text { Dist } \\
(\mathbf{k m})\end{array}$ & $\begin{array}{l}\text { Axim } \\
\text { (deg) }\end{array}$ & $\mathrm{Db}$ & $\begin{array}{l}\text { Tape } \\
\text { Grade }\end{array}$ \\
\hline 142 & 20.82 & 20.9 & 24 & 0 & 228 & 4.29 & 84.5 & 42 & 0 & 269 & 6.88 & 149.8 & 42 & O \\
\hline 148 & 23.93 & 18.1 & 6 & o & 230 & 4.33 & 100.8 & 42 & 0 & 271 & 7.15 & 155.7 & 42 & 0 \\
\hline 151 & 25.48 & 17.0 & 26 & O & 231 & 4.46 & 107.4 & 42 & 0 & 272 & 6.94 & 159.9 & 42 & 0 \\
\hline 154 & 27.04 & 15.9 & 26 & D & 232 & 4.64 & 113.5 & 42 & 0 & 273 & 6.81 & 163.1 & 24 & 0 \\
\hline 157 & 28.59 & 15.0 & 26 & O & 233 & 4.00 & 94.0 & 42 & 0 & 274 & 7.32 & 165.2 & 24 & O \\
\hline 202 & 14.17 & 24.5 & & 3 & 236 & 2.41 & 96.4 & 42 & 0 & 277 & 9.57 & 168.7 & 24 & 0 \\
\hline 203 & 13.67 & 25.4 & 24 & O & 237 & 2.07 & 96.9 & 42 & 0 & 278 & 10.32 & 168.0 & 24 & 0 \\
\hline $\begin{array}{l}204 \\
205\end{array}$ & $\begin{array}{l}13.18 \\
12.71\end{array}$ & 26.4 & 24 & 0 & 238 & 1.32 & 101.7 & 42 & 0 & 278 & 8.05 & 169.7 & 24 & 0 \\
\hline 205 & 12.71 & 27.5 & 24 & 0 & 239 & 0.61 & 115.2 & 42 & 0 & 280 & 9.03 & 183.5 & 24 & 0 \\
\hline 206 & 12.44 & 25.5 & 24 & D & 240 & 0.02 & 348.1 & 42 & 0 & 281 & 8.80 & 182.4 & & 1 \\
\hline 207 & 12.22 & 23.2 & 24 & 0 & 248 & 20.31 & 21.5 & 24 & 0 & 282 & 8.57 & 180.0 & 24 & 0 \\
\hline 213 & 9.56 & 26.5 & 42 & 0 & 254 & 17.31 & 25.5 & & 17 & 288 & 11.57 & 170.7 & 24 & 0 \\
\hline 214 & 9.01 & 28.3 & 42 & 0 & 255 & 16.87 & 26.3 & 24 & 0 & 289 & 12.19 & 171.4 & 24 & 0 \\
\hline 215 & 8.52 & 30.0 & 42 & 0 & 256 & 16.35 & 27.0 & 24 & 0 & 290 & 12.76 & 171.5 & 24 & 0 \\
\hline 216 & 8.09 & 31.8 & 24 & 0 & 257 & 15.91 & 28.0 & 24 & o & 291 & 13.18 & 172.8 & 24 & 0 \\
\hline 217 & 7.67 & 33.8 & 24 & 0 & 258 & 15.48 & 28.8 & 24 & 0 & 292 & 13.65 & 173.5 & 6 & o \\
\hline 218 & 7.21 & 36.2 & 24 & 0 & 259 & 14.92 & 30.1 & 24 & 0 & 293 & 14.27 & 173.3 & 6 & 0 \\
\hline $21 \theta$ & 6.85 & 38.6 & 24 & 0 & 260 & 14.66 & 28.3 & & 25 & 294 & 14.52 & 171.4 & 6 & 0 \\
\hline 220 & 6.40 & 41.8 & & 3 & 261 & 4.97 & 120.7 & 42 & 0 & 295 & 14.78 & 169.6 & 24 & 0 \\
\hline 221 & 5.97 & 45.8 & & 1 & 262 & 4.98 & 122.1 & & 1 & 296 & 15.16 & 168.5 & 24 & 0 \\
\hline 222 & 5.64 & 49.5 & 42 & 0 & 263 & 5.14 & 125.1 & 42 & 0 & 297 & 15.70 & 169.0 & 6 & 25 \\
\hline 223 & 5.30 & 53.9 & 42 & 0 & 264 & 5.49 & 129.4 & 42 & 0 & 298 & 16.14 & 169.5 & & 1 \\
\hline 224 & 4.97 & 59.3 & 42 & O & 265 & 5.77 & 132.5 & 42 & 0 & 299 & 16.60 & 169.2 & 6 & 0 \\
\hline
\end{tabular}


TABLE 5.-Seismic Recorder Data-Continued Shot Number 9 Shot Point 2

Shot Time (Julian day, hr, min, sec): 180:08:00:00.016

\begin{tabular}{|c|c|c|c|c|c|c|c|c|c|c|c|c|c|c|}
\hline Loc & $\begin{array}{l}\text { Dist } \\
(\mathbf{k m})\end{array}$ & $\begin{array}{l}\text { Arim } \\
\text { (deg) }\end{array}$ & $\mathrm{Db}$ & $\begin{array}{l}\text { Tape } \\
\text { Grade }\end{array}$ & Loc & $\begin{array}{c}\text { Dist } \\
(\mathbf{k m})\end{array}$ & $\begin{array}{l}\text { Azim } \\
(\text { deg })\end{array}$ & $\mathrm{Db}$ & $\begin{array}{c}\text { Tape } \\
\text { Grede }\end{array}$ & Loc & $\begin{array}{l}\text { Dist } \\
(\mathbf{k m})\end{array}$ & $\begin{array}{l}\text { Arim } \\
\text { (deg) }\end{array}$ & $\mathrm{Db}$ & $\begin{array}{r}\text { Tape } \\
\text { Grade }\end{array}$ \\
\hline 297 & 44.01 & 185.1 & 6 & 0 & 334 & 55.82 & 185.1 & 6 & 0 & $36 \theta$ & 47.16 & $178 . \theta$ & 6 & 0 \\
\hline 301 & 34.57 & 188.0 & 24 & 15 & 335 & 55.50 & 186.2 & 6 & 0 & 370 & 47.01 & $179 . \theta$ & & 1 \\
\hline 302 & 35.73 & 197.3 & 6 & 0 & 336 & 54.85 & 185.8 & 6 & 15 & 371 & 47.71 & 180.1 & 6 & 0 \\
\hline 303 & 36.32 & 197.1 & & 1 & 337 & 54.27 & 185.2 & 6 & 0 & 372 & 47.99 & 180.7 & 6 & 0 \\
\hline 304 & 37.53 & 197.1 & 6 & 0 & 338 & 53.77 & $185 . \theta$ & 6 & 15 & 373 & 48.82 & 181.1 & 6 & 0 \\
\hline 305 & 38.38 & 186.5 & 24 & $0 / 30$ & 338 & 52.54 & 185.7 & 6 & 0 & 374 & 48.95 & $181 . \theta$ & & $6 / 17$ \\
\hline 306 & 39.56 & 196.2 & & 3 & 340 & 52.21 & 185.9 & 6 & 0 & 375 & 48.14 & 182.1 & 24 & 0 \\
\hline 307 & 40.61 & 195.7 & 6 & 0 & 341 & 33.60 & 177.6 & 6 & 0 & 376 & 48.73 & 183.1 & & $6 / 18$ \\
\hline 308 & 41.36 & 194.9 & 6 & 0 & 342 & 34.69 & 177.6 & 6 & 0 & 377 & 49.44 & 183.0 & 6 & $0 / 31$ \\
\hline 309 & 42.37 & 194.7 & 6 & 15 & 343 & 35.78 & 177.7 & 6 & 0 & 378 & 50.29 & 183.2 & 6 & 0 \\
\hline 310 & 43.42 & 194.6 & 24 & 0 & 344 & 36.75 & 177.8 & 6 & 0 & 379 & 50.35 & 184.1 & 6 & 0 \\
\hline 311 & 44.14 & 194.3 & 6 & 0 & 345 & 37.74 & $17 \pi .8$ & 6 & 0 & 380 & 50.67 & 184.8 & & 12 \\
\hline 312 & 39.42 & $194 . \theta$ & 42 & 15 & 346 & 38.85 & 177.0 & 6 & 0 & 381 & 71.62 & 188.6 & 6 & 0 \\
\hline 313 & 39.24 & 193.1 & 42 & $0 / 17$ & 347 & 39.80 & 178.0 & 6 & 0 & 382 & 71.40 & $187 . \theta$ & 6 & 0 \\
\hline 314 & 38.84 & 181.1 & 24 & 0 & 348 & 40.77 & 178.4 & 6 & 0 & 383 & 70.65 & 188.1 & 6 & 0 \\
\hline 315 & 39.86 & 189.6 & 6 & 0 & 349 & 42.03 & 178.4 & 6 & 0 & 384 & 69.39 & 187.7 & 6 & 0 \\
\hline 316 & 40.40 & 188.4 & 6 & 0 & $\mathbf{3 5 0}$ & 43.12 & 178.6 & 6 & 15 & 385 & 68.83 & 187.0 & & 15 \\
\hline 317 & 41.20 & $185 . \theta$ & 6 & 0 & 351 & 43.66 & 178.1 & 6 & 0 & 386 & 68.42 & 186.8 & 36 & 12 \\
\hline 318 & 41.51 & 184.8 & 42 & 15 & 352 & 42.84 & 179.6 & 6 & 0 & 387 & 67.87 & 186.6 & 6 & 0 \\
\hline 310 & 41.30 & 183.7 & 6 & 0 & 353 & 42.49 & 181.2 & 6 & 0 & 388 & 67.42 & 186.4 & 6 & 0 \\
\hline 320 & 41.97 & 182.7 & 6 & 0 & 355 & 44.43 & 184.1 & & 15 & 389 & 66.09 & 186.2 & 24 & 0 \\
\hline 321 & 62.89 & 187.0 & & 17 & 356 & 44.74 & 183.5 & 6 & 15 & 380 & 66.69 & 186.7 & 6 & 0 \\
\hline 322 & 62.58 & 187.2 & 6 & 0 & 357 & 45.00 & 182.3 & & 17 & 391 & 66.38 & 186.8 & & 3 \\
\hline 323 & 61.70 & 187.2 & 24 & 12 & 358 & 45.13 & 181.5 & 6 & 0 & 392 & 65.94 & 186.8 & 24 & 12 \\
\hline 324 & 61.31 & 186.8 & 24 & 12 & 359 & 45.35 & 180.6 & 6 & 0 & 393 & 65.50 & 186.6 & 24 & 12 \\
\hline 325 & 60.87 & 186.8 & 6 & 0 & 360 & 45.39 & 179.7 & 6 & 0 & 394 & 65,16 & 186.1 & 6 & 0 \\
\hline 326 & 60.38 & 186.5 & 6 & 0 & 361 & 45.68 & 179.5 & 6 & 0 & 395 & 64.45 & 185.8 & 24 & 12 \\
\hline 327 & 59.91 & 185.6 & 6 & 0 & 362 & 46.02 & 179.2 & 6 & 25 & 396 & 64.01 & 185.6 & 6 & 0 \\
\hline 328 & 59.05 & 186.0 & 6 & 0 & 363 & 46.44 & 178.9 & 6 & 0 & 397 & 63.68 & 185.4 & 6 & 0 \\
\hline $32 \theta$ & 58.31 & $185 . \theta$ & 6 & 0 & 364 & 46.62 & 178.4 & 6 & 0 & 398 & 63.35 & 185.5 & 24 & 12 \\
\hline 330 & 58.02 & 185.4 & 24 & 12 & 365 & 46.90 & 178.0 & 6 & 0 & 399 & 63.08 & 185.6 & 24 & 12 \\
\hline 331 & 57.75 & 185.7 & 24 & 15 & 366 & 47.27 & 177.1 & 6 & 0 & 400 & 62.81 & 186.0 & 6 & 0 \\
\hline 332 & 57.25 & 185.7 & 6 & 0 & 367 & 47.46 & 176.8 & 6 & 0 & & & & & \\
\hline 333 & 56.55 & 184.9 & 6 & 0 & 368 & 47.36 & 178.0 & 6 & 0 & & & & & \\
\hline
\end{tabular}


TABLE 5.-Seismic Recorder Data-Continued Shot Number 10 Shot Point 10

Shot Time (Julian day, hr, min, sec): 114:20:01:13.785

\begin{tabular}{|c|c|c|c|c|c|c|c|c|c|c|c|c|c|c|}
\hline Loc & $\begin{array}{c}\text { Dist } \\
(\mathbf{k m})\end{array}$ & $\begin{array}{l}\text { Azim } \\
\text { (deg) }\end{array}$ & $\mathrm{Db}$ & $\begin{array}{l}\text { Tepe } \\
\text { Grede }\end{array}$ & Loc & $\begin{array}{c}\text { Dist } \\
(\mathbf{k m})\end{array}$ & $\begin{array}{l}\text { Azim } \\
\text { (deg) }\end{array}$ & $\mathbf{D b}$ & $\begin{array}{l}\text { Tepe } \\
\text { Grede }\end{array}$ & Loc & $\begin{array}{c}\text { Dist } \\
(\mathbf{k m})\end{array}$ & $\begin{array}{l}\text { Arim } \\
(\text { deg })\end{array}$ & $\mathrm{Db}$ & $\begin{array}{r}\text { Tepe } \\
\text { Grade }\end{array}$ \\
\hline 107 & 16.77 & 205.5 & 42 & $0 / 30$ & 286 & 67.61 & 192.2 & & 1 & 468 & 4.83 & 187.3 & 82 & 0 \\
\hline 110 & 18.09 & 206.8 & 42 & 0 & 289 & 68.96 & 192.1 & 42 & 0 & 469 & 3.05 & 177.0 & 82 & $0 / 15 / 30$ \\
\hline 113 & 19.57 & 206.2 & 42 & 0 & 291 & 69.99 & 192.0 & & 15 & 470 & 2.57 & 176.2 & 62 & 0 \\
\hline 116 & 21.02 & 204.3 & 42 & 0 & 293 & 71.06 & 191.8 & 24 & 0 & 471 & 1.75 & 174.3 & & 1 \\
\hline 119 & 22.40 & 202.7 & 42 & 0 & 296 & 71.47 & 190.7 & 24 & $0 / 17$ & 472 & 1.25 & 190.5 & 82 & 0 \\
\hline 147 & 34.33 & 194.8 & 24 & 0 & 299 & 72.88 & 190.4 & 24 & 0 & 473 & 0.00 & 238.7 & 94 & 0 \\
\hline 150 & 32.76 & 195.5 & & 25 & 401 & 35.00 & 194.3 & & 15 & 474 & 1.00 & 33.8 & 62 & 13 \\
\hline 153 & 31.19 & 196.3 & & 17 & 441 & 74.72 & 10.2 & 24 & 0 & 475 & 2.55 & 352.3 & 62 & 0 \\
\hline 156 & 29.65 & 197.2 & 24 & 15 & 442 & 72.63 & 10.5 & 42 & 0 & 476 & 4.06 & 354.3 & & 28 \\
\hline 159 & 28.08 & 198.2 & 42 & 15 & 443 & 71.53 & 10.6 & & 9 & 477 & 5.94 & 354.3 & & 28 \\
\hline 183 & 26.63 & 199.3 & & 3 & 444 & 70.48 & 10.8 & 42 & 0 & 478 & 8.19 & 358.4 & 42 & 0 \\
\hline 194 & 25.17 & 200.3 & & 6 & 445 & 67.77 & 11.7 & & 1 & 479 & 10.09 & 4.8 & 42 & 0 \\
\hline 198 & 23.98 & 201.4 & & 1 & 446 & 66.33 & 12.4 & 24 & 15 & 480 & 11.35 & 6.6 & & 25 \\
\hline 203 & 44.27 & 193.5 & 42 & 15 & 447 & 64.39 & 12.5 & 24 & 0 & 481 & 47.13 & 10.0 & 42 & 0 \\
\hline 210 & 46.78 & 194.8 & 42 & 0 & 448 & 62.91 & 12.0 & 42 & 0 & 482 & 46.20 & 10.6 & 24 & 0 \\
\hline 213 & 48.33 & 194.3 & 42 & 15 & 449 & 61.43 & 11.6 & & 1 & 483 & 45.12 & 10.3 & 42 & 0 \\
\hline 216 & 49.96 & 193.9 & 42 & 0 & 450 & 59.76 & 11.0 & 24 & 0 & 484 & 43.93 & 10.4 & 42 & 0 \\
\hline 210 & 51.44 & 193.4 & & 17 & 451 & 58.77 & 10.3 & & 1 & 485 & 42.46 & 10.6 & 42 & 0 \\
\hline 222 & 53.08 & 193.0 & & 1 & 452 & 56.74 & $\mathbf{9 . 3}$ & 24 & 0 & 486 & 40.30 & 10.7 & 42 & 0 \\
\hline 225 & 54.64 & 192.6 & 42 & 0 & 453 & 55.09 & 0.1 & 24 & 15 & 487 & 38.50 & 10.6 & 42 & 0 \\
\hline 228 & 56.25 & 192.3 & & 3 & 454 & 53.65 & 9.2 & 24 & $\mathbf{0}$ & 488 & 36.90 & 10.1 & 24 & 0 \\
\hline 231 & 57.97 & 191.9 & & 3 & 455 & 52.43 & 9.3 & 24 & 0 & 489 & 35.73 & 6.4 & 42 & 0 \\
\hline 248 & 37.53 & 193.5 & 24 & 0 & 456 & 51.31 & 9.3 & & 17 & 490 & 33.62 & 7.4 & 42 & 0 \\
\hline 251 & 39.13 & 192.9 & 24 & 0 & 457 & 49.88 & 9.4 & & 28 & 491 & 31.64 & 7.8 & 42 & 0 \\
\hline 254 & 40.72 & 192.4 & 42 & 0 & 458 & 48.92 & 9.4 & 24 & 0 & 492 & 29.77 & 7.0 & 42 & 0 \\
\hline 257 & 42.26 & 192.0 & 42 & $\mathbf{0}$ & 459 & 47.95 & 9.5 & 24 & $\mathbf{0}$ & 493 & 27.85 & 6.0 & & 1 \\
\hline 260 & 43.48 & 192.3 & 42 & 0 & 461 & 15.51 & 208.8 & 42 & 0 & 494 & 25.54 & 4.1 & 42 & 0 \\
\hline 262 & 59.26 & 191.7 & 42 & 0 & 462 & 14.14 & 205.7 & 42 & 0 & 495 & 23.89 & 4.3 & 42 & 0 \\
\hline 265 & 60.48 & 191.4 & 42 & 0 & 463 & 12.80 & 205.3 & 42 & 0 & 496 & 21.63 & 4.8 & 42 & 15 \\
\hline 268 & 62.01 & 191.9 & 42 & 0 & 464 & 11.21 & 206.7 & 42 & 0 & 497 & 19.90 & 5.3 & 42 & 0 \\
\hline 273 & 63.51 & 193.0 & 42 & 0 & 465 & 9.46 & 213.1 & 42 & 0 & 498 & 17.27 & 3.6 & 42 & 9 \\
\hline 277 & 66.33 & 192.5 & 24 & 0 & 466 & 7.74 & 207.4 & 42 & $0 / 30$ & 499 & 14.93 & 4.0 & & 1 \\
\hline $27 \theta$ & 65.01 & 193.2 & 42 & 0 & 467 & 6.65 & 201.3 & 42 & 0 & 500 & 13.41 & 7.4 & & 3 \\
\hline
\end{tabular}


TABLE 5.-Seismic Recorder Data-Continued Shot Number 11 Shot Point 11

Shot Time (Julian day, hr, min, sec): 114:20:02:23.085

\begin{tabular}{|c|c|c|c|c|c|c|c|c|c|c|c|c|c|c|}
\hline Loc & $\begin{array}{c}\text { Dist } \\
(\mathbf{k m})\end{array}$ & $\begin{array}{l}\text { Axim } \\
\text { (deg) }\end{array}$ & $\mathbf{D b}$ & $\begin{array}{l}\text { Tepe } \\
\text { Grede }\end{array}$ & Loc & $\begin{array}{l}\text { Dist } \\
(\mathbf{k m})\end{array}$ & $\begin{array}{l}\text { Azim } \\
\text { (deg) }\end{array}$ & $\mathrm{Db}$ & $\begin{array}{l}\text { Tepe } \\
\text { Grede }\end{array}$ & Loc & $\begin{array}{l}\text { Dist } \\
(\mathbf{k m})\end{array}$ & $\begin{array}{l}\text { Azim } \\
\text { (deg) }\end{array}$ & $\mathrm{Db}$ & $\begin{array}{r}\text { Tape } \\
\text { Grade }\end{array}$ \\
\hline 107 & 16.77 & 204.5 & 42 & 0 & 286 & 67.68 & 191.9 & & 1 & 468 & 4.93 & 184.1 & 42 & 0 \\
\hline 110 & 18.08 & 205.9 & 42 & 0 & 289 & 69.02 & 191.9 & 24 & 0 & 469 & 3.19 & 172.4 & 62 & 0 \\
\hline 113 & 19.57 & 205.4 & 42 & 0 & 291 & 70.05 & 191.8 & & 12 & 470 & 2.72 & 170.8 & 62 & 0 \\
\hline 116 & 21.03 & 203.5 & 42 & 0 & 293 & 71.13 & 191.6 & 24 & 0 & 471 & 1.91 & 166.8 & & 1 \\
\hline 119 & 22.42 & 202.0 & 24 & 0 & 296 & 71.54 & 190.4 & & 12 & 472 & 1.35 & 178.6 & 82 & 0 \\
\hline 147 & 34.38 & 194.3 & 24 & 0 & 299 & 72.95 & 190.2 & 24 & 0 & 473 & 0.29 & 114.8 & 94 & 0 \\
\hline 150 & 32.81 & 195.0 & & 25 & 401 & 35.06 & 193.9 & & 25 & 474 & 1.09 & 49.0 & 62 & 0 \\
\hline 153 & 31.24 & 195.8 & & 17 & 441 & 74.65 & 10.4 & 6 & 25 & 475 & 2.41 & 358.1 & 62 & 0 \\
\hline 156 & 29.69 & 196.7 & 24 & 0 & 442 & 72.56 & 10.7 & 24 & 0 & 476 & 3.92 & 358.0 & & 28 \\
\hline 159 & 28.11 & 197.6 & 24 & 0 & 443 & 71.46 & 10.9 & & 9 & 477 & 5.80 & 356.7 & & 28 \\
\hline 183 & 26.56 & 198.7 & & 3 & 444 & 70.41 & 11.0 & 24 & 0 & 478 & 8.07 & 0.2 & 42 & 0 \\
\hline 194 & 25.20 & 199.7 & & 6 & 445 & 67.71 & 12.0 & & 1 & 479 & 10.00 & 6.4 & 42 & 0 \\
\hline 198 & 23.99 & 200.8 & & 1 & 446 & 66.27 & 12.7 & 24 & 25 & 480 & 11.27 & 8.0 & & 25 \\
\hline 203 & 44.32 & 193.2 & 24 & 0 & 447 & 64.33 & 12.7 & 24 & 0 & 481 & 47.06 & 10.3 & 24 & 0 \\
\hline 210 & 46.83 & 194.5 & 24 & 0 & 448 & 62.85 & 12.3 & 24 & 0 & 482 & 46.13 & 10.9 & 24 & 0 \\
\hline 213 & 48.38 & 194.0 & 24 & 0 & 449 & 61.36 & 11.9 & & 1 & 483 & 45.05 & 10.6 & 42 & 0 \\
\hline 216 & 50.01 & 193.5 & 24 & 0 & 450 & 59.69 & 11.2 & 24 & 16 & 484 & 43.86 & 10.7 & 42 & 0 \\
\hline 219 & 51.50 & 193.1 & & $14 / 17$ & 451 & 58.70 & 10.6 & & 1 & 485 & 42.39 & 11.0 & 24 & $\mathbf{0}$ \\
\hline 222 & 53.14 & 192.7 & & 1 & 452 & 56.66 & 9.6 & 24 & 0 & 486 & 40.23 & 11.0 & 42 & 0 \\
\hline 225 & 54.70 & 192.3 & 24 & 0 & 453 & 55.01 & 9.4 & 24 & 0 & 487 & 38.44 & 11.0 & 24 & 0 \\
\hline 228 & 56.32 & 192.0 & & 3 & 454 & 53.57 & 9.5 & 24 & 0 & 488 & 36.82 & 10.5 & 24 & 0 \\
\hline 231 & 58.03 & 191.7 & & 3 & 455 & 52.35 & 9.6 & 24 & 0 & 489 & 35.64 & 6.8 & 24 & 0 \\
\hline 248 & 37.59 & 193.1 & 24 & 0 & 456 & 51.23 & 9.6 & & 17 & 490 & 33.54 & 7.8 & 24 & 0 \\
\hline 251 & 39.19 & 192.5 & 24 & 0 & 457 & 49.81 & 9.7 & & 28 & 491 & 31.56 & 8.3 & 24 & 0 \\
\hline 254 & 40.78 & 192.0 & 24 & 0 & 458 & 48.85 & 9.8 & 24 & 0 & 492 & 29.68 & 7.5 & 24 & 0 \\
\hline 257 & 42.32 & 191.6 & 24 & 0 & 459 & 47.87 & 9.9 & 24 & 0 & 493 & 27.75 & 6.5 & & 1 \\
\hline 260 & 43.54 & 191.9 & & 3 & 461 & 15.50 & 207.8 & 42 & 0 & 494 & 25.44 & 4.7 & 42 & 0 \\
\hline 262 & 59.33 & 191.4 & 42 & 0 & 462 & 14.14 & 204.6 & 42 & 0 & 495 & 23.79 & 4.9 & 42 & 0 \\
\hline 265 & 60.55 & 191.2 & 24 & 0 & 463 & 12.80 & 204.0 & 42 & 0 & 496 & 21.54 & 5.6 & 42 & 0 \\
\hline 268 & 62.07 & 191.6 & & $9 / 17$ & 464 & 11.21 & 205.2 & 42 & 0 & 497 & 19.80 & 6.1 & & 12 \\
\hline 273 & 63.57 & 192.7 & 24 & $0 / 15$ & 465 & 9.38 & 211.2 & 42 & 0 & 498 & 17.17 & 4.5 & & 29 \\
\hline 277 & 66.39 & 192.3 & 24 & 0 & 466 & 7.73 & 205.2 & 42 & 0 & 499 & 14.83 & 5.1 & & 1 \\
\hline 279 & 65.06 & 192.9 & 24 & 0 & 467 & 6.68 & 198.9 & 42 & 0 & 500 & 13.33 & 8.6 & & 3 \\
\hline
\end{tabular}


TABLE 5.-Seismic Recorder Data-Continued

Shot Number 12 Shot Point 12

Shot Time (Julian day, hr, min, sec): 115:21:00:58.487

\begin{tabular}{|c|c|c|c|c|c|c|c|c|c|c|c|c|c|c|}
\hline Loc & $\begin{array}{l}\text { Dist } \\
(\mathbf{k m})\end{array}$ & $\begin{array}{l}\text { Azim } \\
\text { (deg) }\end{array}$ & $\mathrm{Db}$ & $\begin{array}{l}\text { Tepe } \\
\text { Grede }\end{array}$ & Loc & $\begin{array}{c}\text { Dist } \\
(\mathbf{k m})\end{array}$ & $\begin{array}{l}\text { Arim } \\
\text { (deg) }\end{array}$ & Db & $\begin{array}{l}\text { Tape } \\
\text { Grade }\end{array}$ & Loc & $\begin{array}{c}\text { Dist } \\
(\mathbf{k m})\end{array}$ & $\begin{array}{l}\text { Arim } \\
\text { (deg) }\end{array}$ & $\mathbf{D b}$ & $\begin{array}{l}\text { Tepe } \\
\text { Grede }\end{array}$ \\
\hline 107 & 91.22 & 193.1 & 24 & 0 & 286 & 142.53 & 191.2 & 24 & 15 & 468 & 79.77 & 190.1 & & 3 \\
\hline 110 & 92.42 & 193.5 & & 1 & 289 & 143.88 & 191.2 & 24 & 0 & 469 & 77.91 & 189.8 & & 3 \\
\hline 113 & 93.91 & 193.6 & 24 & 0 & 291 & 144.91 & 191.2 & & 12 & 470 & 77.44 & 189.8 & & 3 \\
\hline 116 & 95.47 & 193.4 & & 1 & 293 & 145.99 & 191.1 & 6 & 0 & 471 & 76.62 & 189.9 & & 3 \\
\hline 119 & 96.93 & 193.2 & 24 & 0 & 296 & 146.41 & 190.5 & & 12 & 472 & 76.19 & 190.3 & & 3 \\
\hline 147 & 109.19 & 191.7 & 24 & 0 & 299 & 147.82 & 190.4 & 6 & 0 & 473 & 74.94 & 190.3 & & 3 \\
\hline 150 & 107.60 & 191.9 & & 25 & 401 & 109.88 & 191.6 & & 25 & 474 & 74.02 & 190.0 & & 3 \\
\hline 153 & 106.01 & 192.1 & 6 & 0 & 441 & 0.22 & 188.6 & 94 & 0 & 475 & 72.52 & 190.9 & & 3 \\
\hline 156 & 104.43 & 192.3 & 24 & 15 & 442 & 2.33 & 182.4 & 94 & 0 & 476 & 71.05 & 191.2 & & 28 \\
\hline $15 \theta$ & 102.82 & 192.5 & 24 & 0 & 443 & 3.45 & 181.5 & & 9 & 477 & 69.24 & 191.6 & & 28 \\
\hline 183 & 101.22 & 192.7 & & 17 & 444 & 4.52 & 181.4 & 42 & 0 & 478 & 66.94 & 191.7 & & 3 \\
\hline 194 & $\mathbf{9 9 . 8 2}$ & 192.8 & 6 & 6 & 445 & 7.41 & 176.1 & 42 & 0 & 479 & 64.90 & 191.1 & & 3 \\
\hline 198 & 98.57 & 193.0 & & 1 & 446 & $\boldsymbol{9 . 0 3}$ & 173.8 & & 1 & 480 & 63.61 & 190.9 & & 3 \\
\hline 203 & 118.16 & 191.5 & 24 & 15 & 447 & 10.90 & 176.8 & 42 & 0 & 481 & 27.81 & 190.7 & 42 & 0 \\
\hline 210 & 121.62 & 192.1 & 24 & 0 & 448 & 12.23 & 180.8 & 42 & 0 & 482 & 28.75 & 189.8 & 24 & 0 \\
\hline 213 & 123.19 & 191.9 & 24 & 15 & 449 & 13.62 & 183.9 & 42 & 0 & 483 & 29.81 & 190.3 & 24 & 0 \\
\hline 216 & 124.84 & 191.8 & 24 & 0 & 450 & 15.21 & 187.3 & 42 & 0 & 484 & 31.01 & 190.0 & 42 & 0 \\
\hline $21 \theta$ & 126.33 & 191.6 & 24 & 0 & 451 & 16.17 & 189.8 & 42 & 0 & 485 & 32.48 & 189.8 & 24 & 0 \\
\hline 222 & 127.98 & 191.5 & & 1 & 452 & 18.23 & 193.1 & 42 & 0 & 486 & 34.64 & 189.8 & 24 & 0 \\
\hline 225 & 129.55 & 191.3 & 24 & 0 & 453 & 19.88 & 193.2 & 42 & 0 & 487 & 36.44 & 189.9 & 24 & 0 \\
\hline 228 & 131.17 & 191.2 & & 3 & 454 & 21.32 & 192.7 & 42 & 0 & 488 & 38.04 & 180.4 & 24 & 0 \\
\hline 231 & 132.89 & 191.0 & & 3 & 456 & 23.65 & 192.2 & 42 & 0 & 489 & 39.36 & 193.8 & 24 & 0 \\
\hline 248 & 112.43 & 191.4 & 24 & 0 & 457 & 25.07 & 191.9 & & 28 & 490 & 41.39 & 192.6 & & 1 \\
\hline 251 & 114.05 & 191.2 & 24 & 0 & 458 & 26.03 & 191.8 & 24 & 0 & 491 & 43.34 & 192.0 & 24 & 0 \\
\hline 254 & 115.64 & 191.1 & 24 & 0 & 459 & 27.00 & 191.5 & 42 & 0 & 492 & 45.25 & 192.4 & 24 & 0 \\
\hline 257 & 117.18 & 190.9 & 24 & 0 & 460 & 1.38 & 181.7 & 82 & 0 & 493 & 47.21 & 192.8 & & 1 \\
\hline 260 & 118.40 & 191.1 & 24 & 0 & 461 & 89.78 & 193.5 & & 3 & 494 & 49.62 & 193.4 & 24 & 0 \\
\hline 262 & 134.19 & 191.0 & 24 & 0 & 462 & 88.64 & 192.7 & & 3 & 495 & 51.24 & 193.0 & 24 & 0 \\
\hline 265 & 135.41 & 190.8 & 24 & 0 & 463 & 87.36 & 192.5 & & 3 & 496 & 53.44 & 192.5 & 24 & 0 \\
\hline 268 & 136.93 & 191.1 & 24 & 0 & 464 & 85.75 & 192.4 & & 3 & 497 & 55.14 & 192.1 & 24 & 0 \\
\hline 273 & 138.41 & 191.6 & & 15 & 465 & 83.71 & 192.8 & & 3 & 498 & 57.82 & 192.3 & & 29 \\
\hline 277 & 141.24 & 191.4 & 6 & 0 & 466 & 82.37 & 191.9 & & 3 & 499 & 60.12 & 191.8 & & 1 \\
\hline 279 & 139.90 & 191.7 & 24 & 0 & 467 & 81.48 & 191.2 & & 3 & 500 & 61.55 & $190 . \theta$ & 24 & 0 \\
\hline
\end{tabular}


TABle 5.-Seismic Recorder Data-Continued

Shot Number 19 Shot Point 13

Shot Time (Julian day, hr, min, sec): 312:20:00:00.190

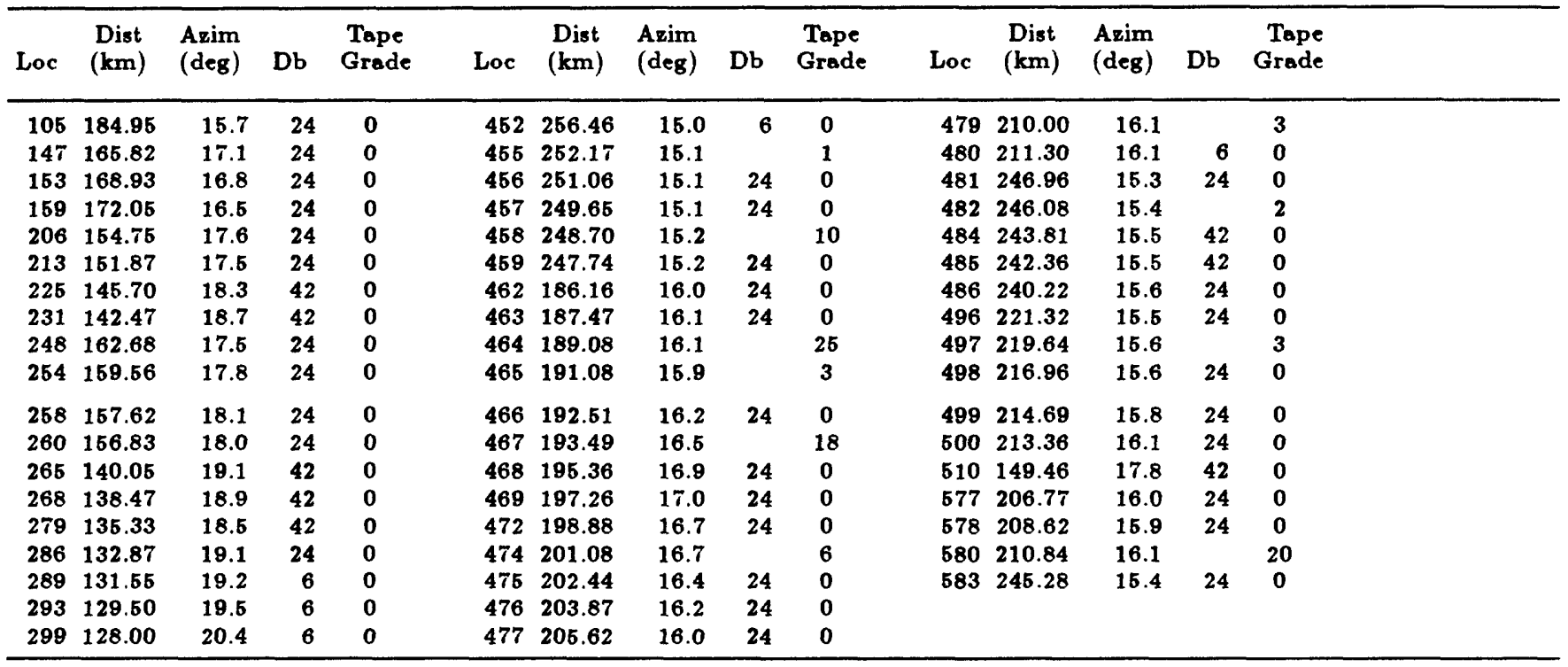




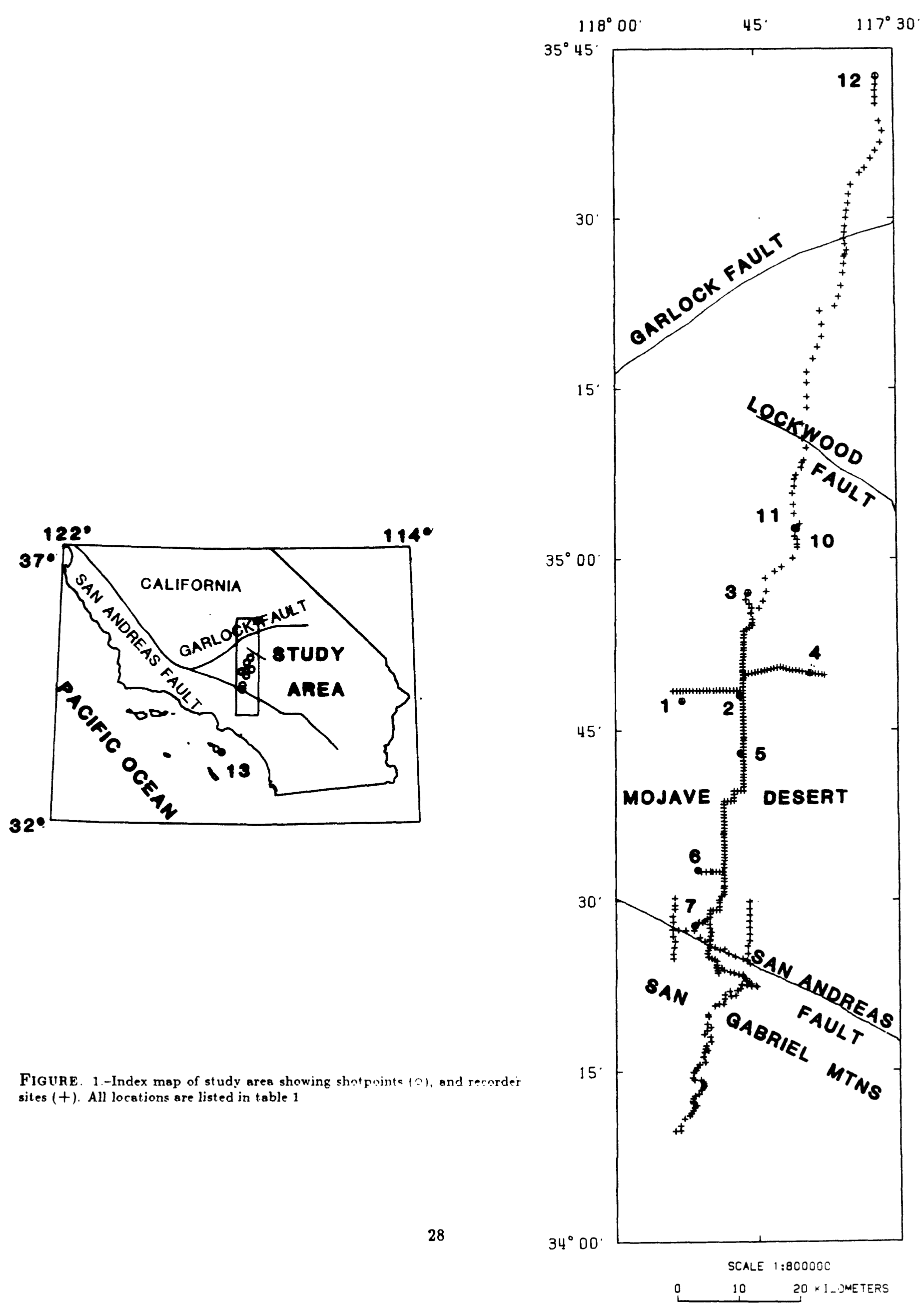




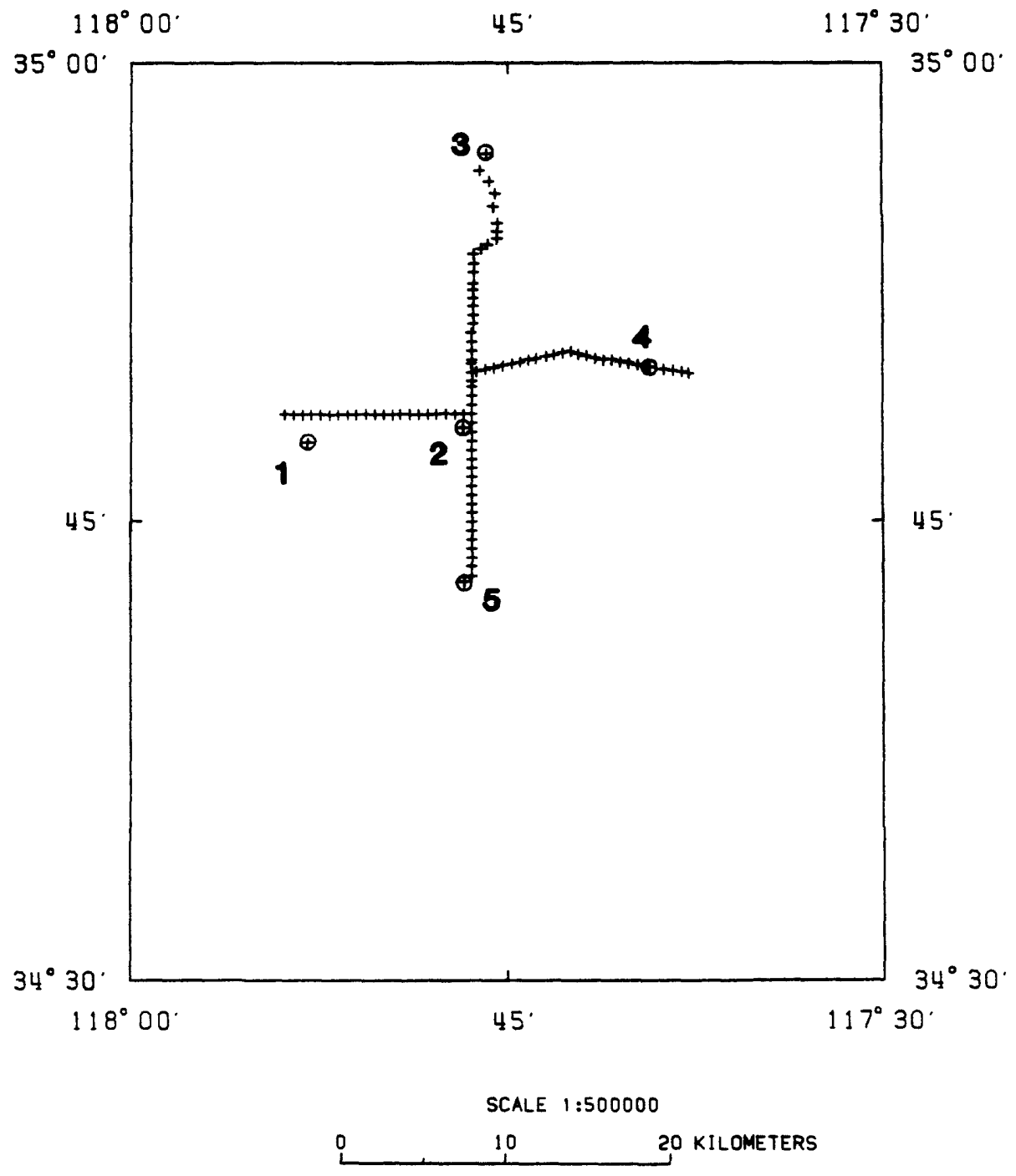

Figure 2.-Location of recorder sites $(+)$ and shotpoints $(c)$ for deployment 1 , shot numberc 1-5 (stations 101 - 200). 


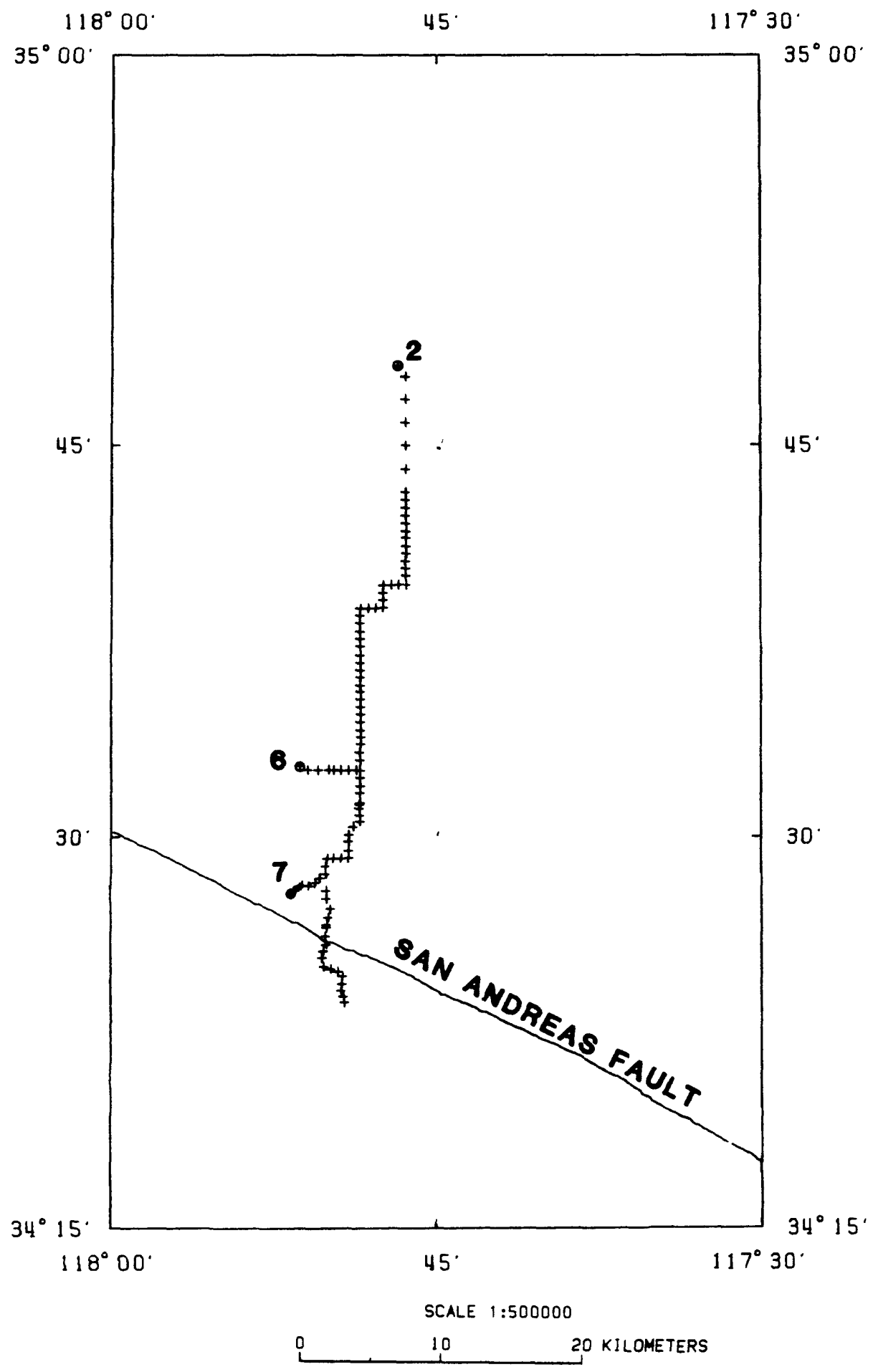

Figure 3.-Location of recorder sites $(+)$ and shotpoints $(0)$ for deployment 2, shot numbers 6-8 (stations 142 $-300)$. 


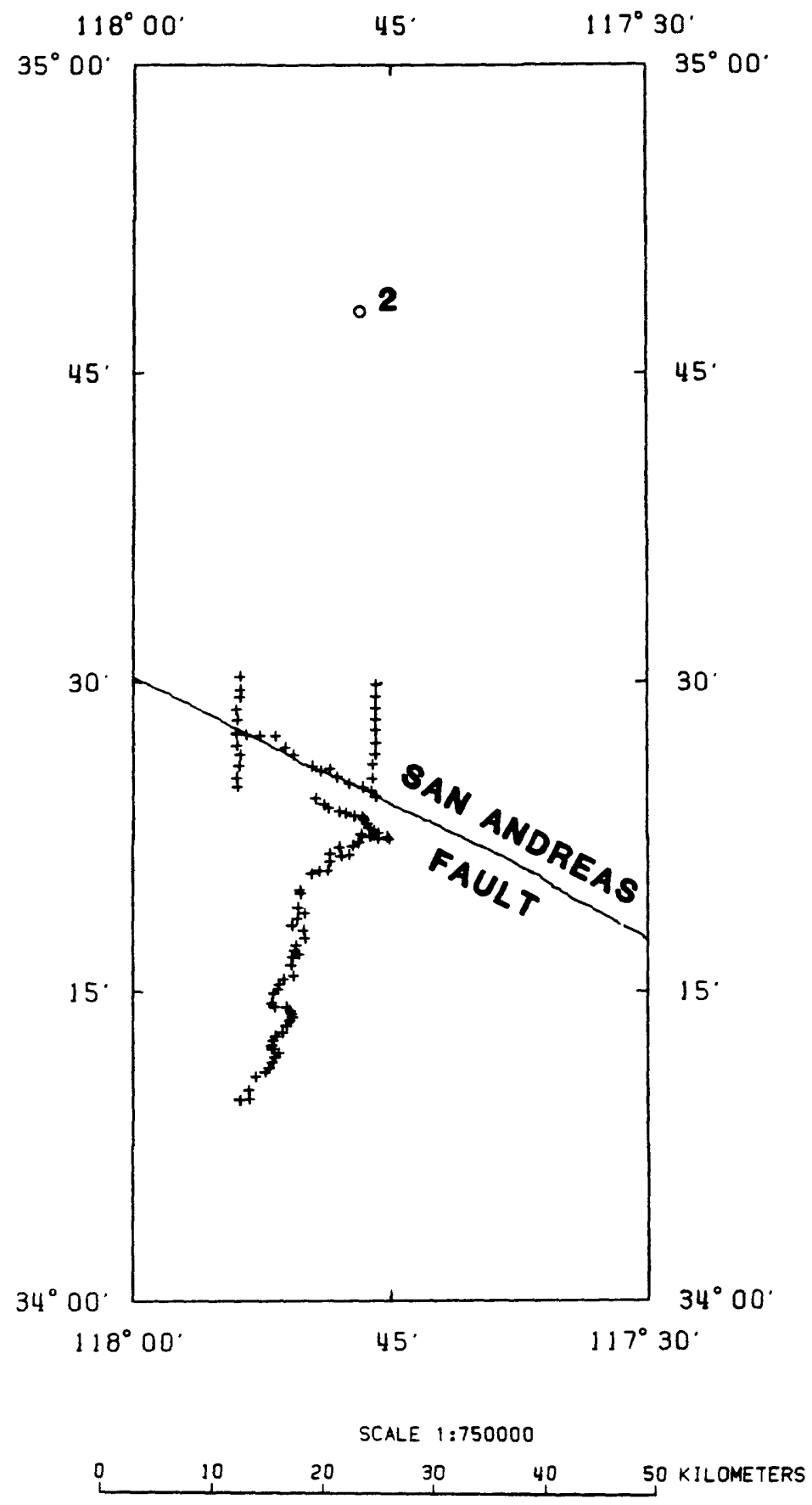

Figure. 4.-Location of recorder sites $(+)$ and shotpoint $(0)$ for deployment 3 , shot mumlier 9 (stations 297 . 400). 


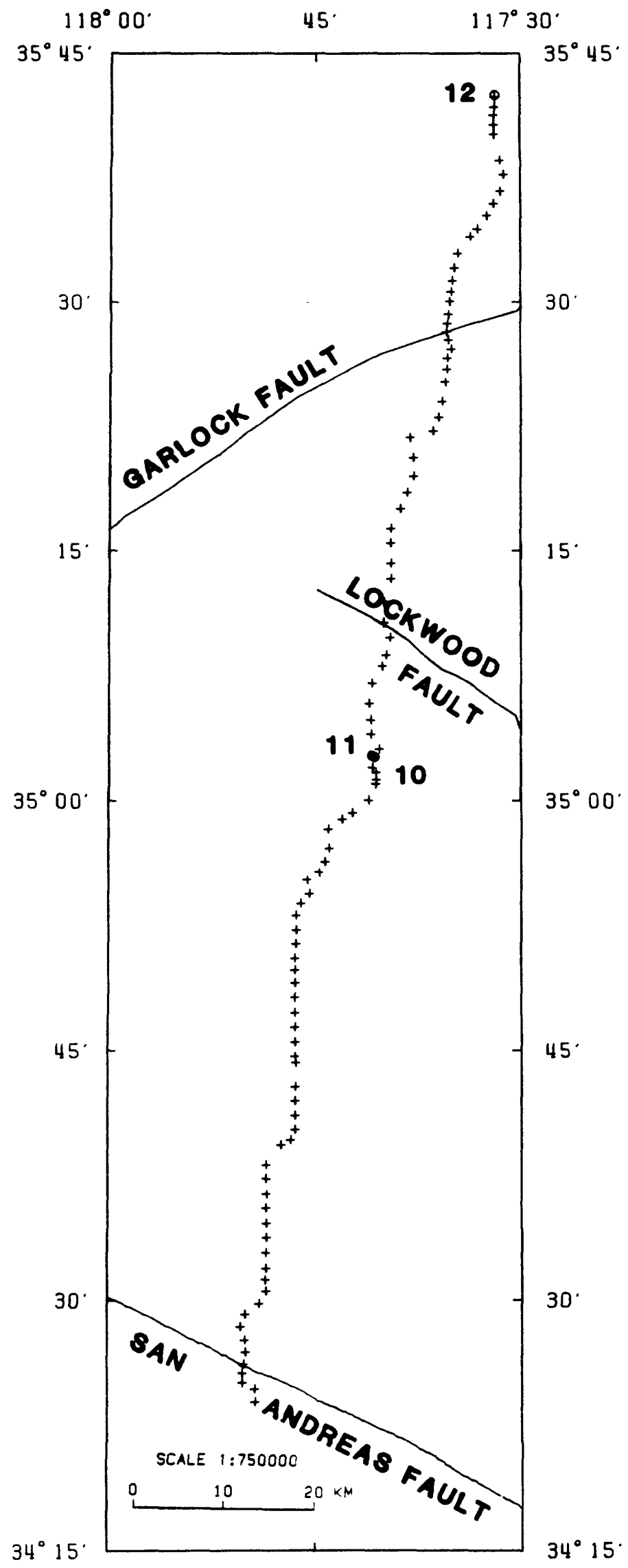

Figure. 5.-Location of recorder sites $(+)$ and shotpoint $(0)$ for deployment 4, shot numlers 10-12 (stations $107-500)$. 


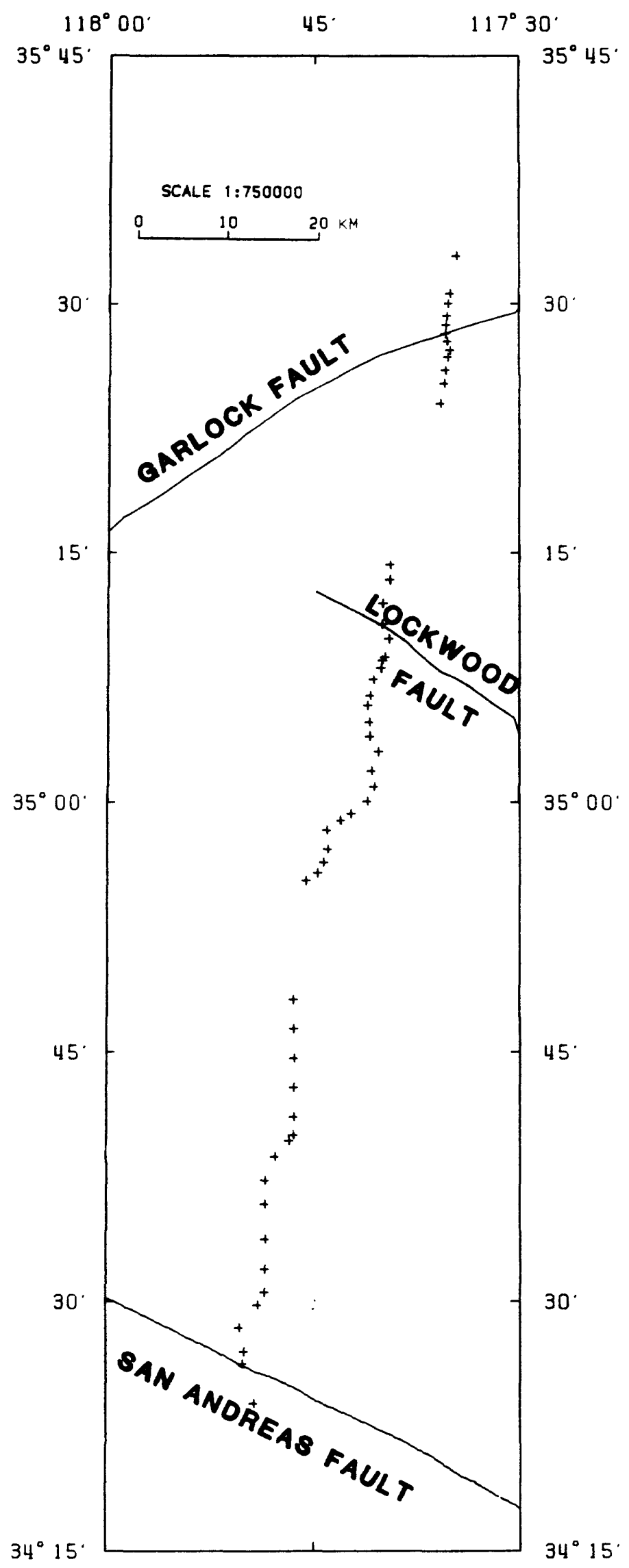

FiguRE 6.-Location of recorder sites $(+)$ for deployment 5 , shotnumber 13 (statione 105 - 5.83). The shot point was located on Catalina Island, not shown. 


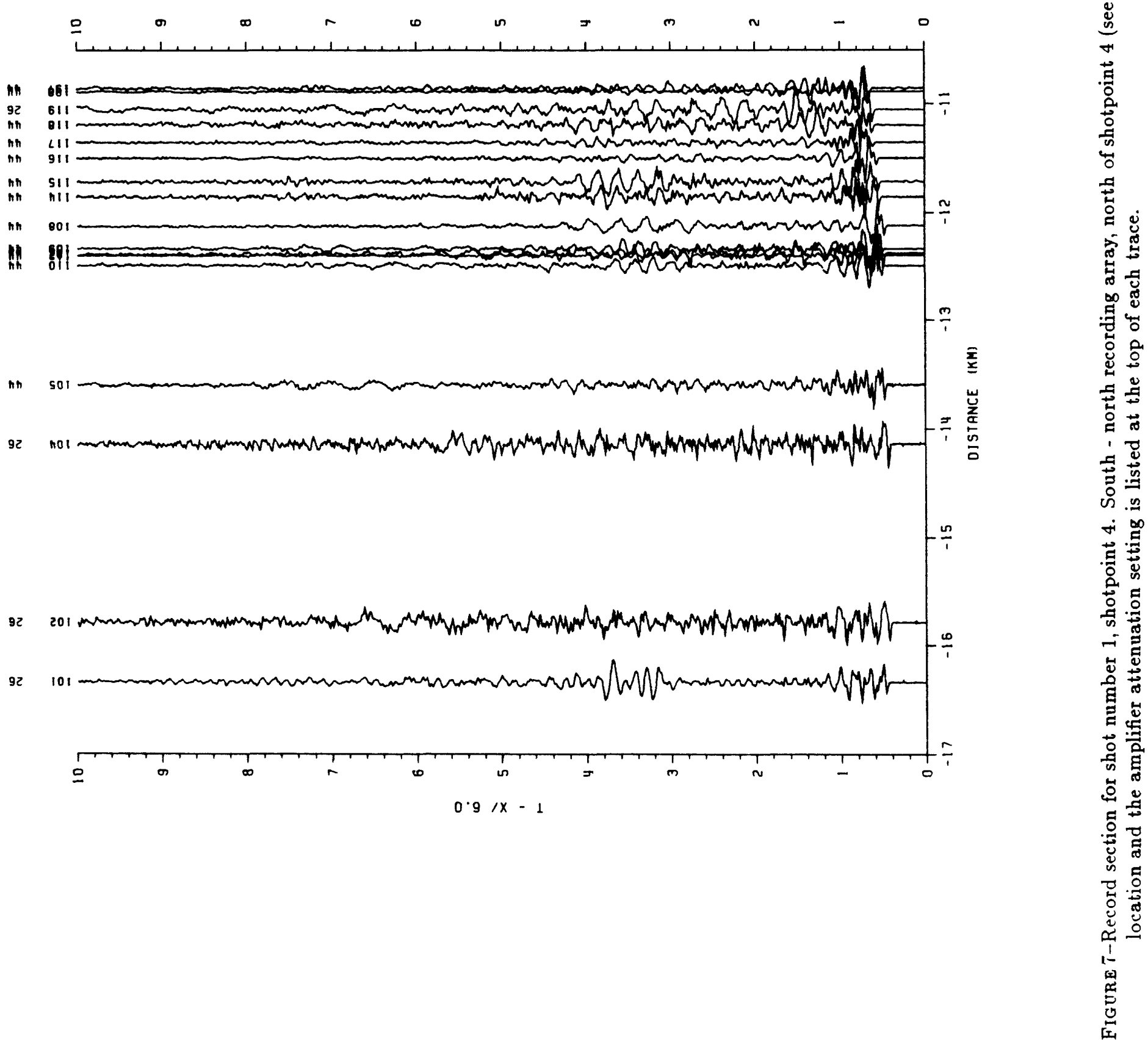




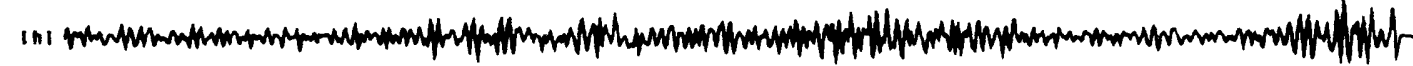

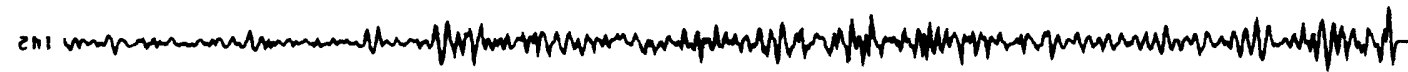

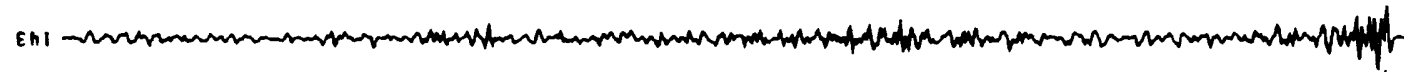
thi -

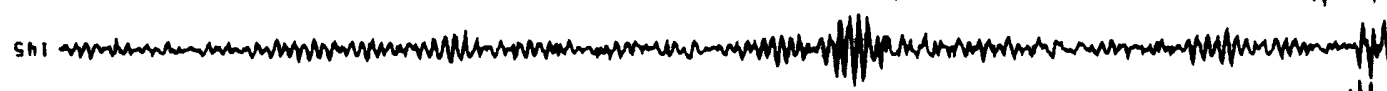
$9 n 1$ Lht

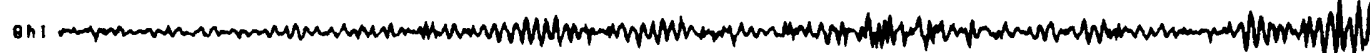
6 on osi 1 $|S|$ 烈

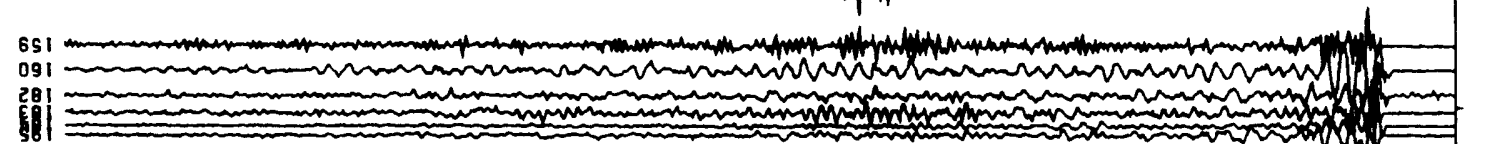

E61

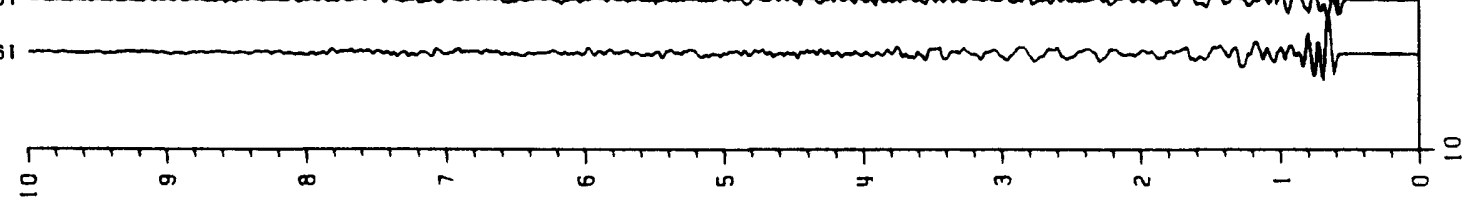

$0 \cdot 9 / x-1$ 


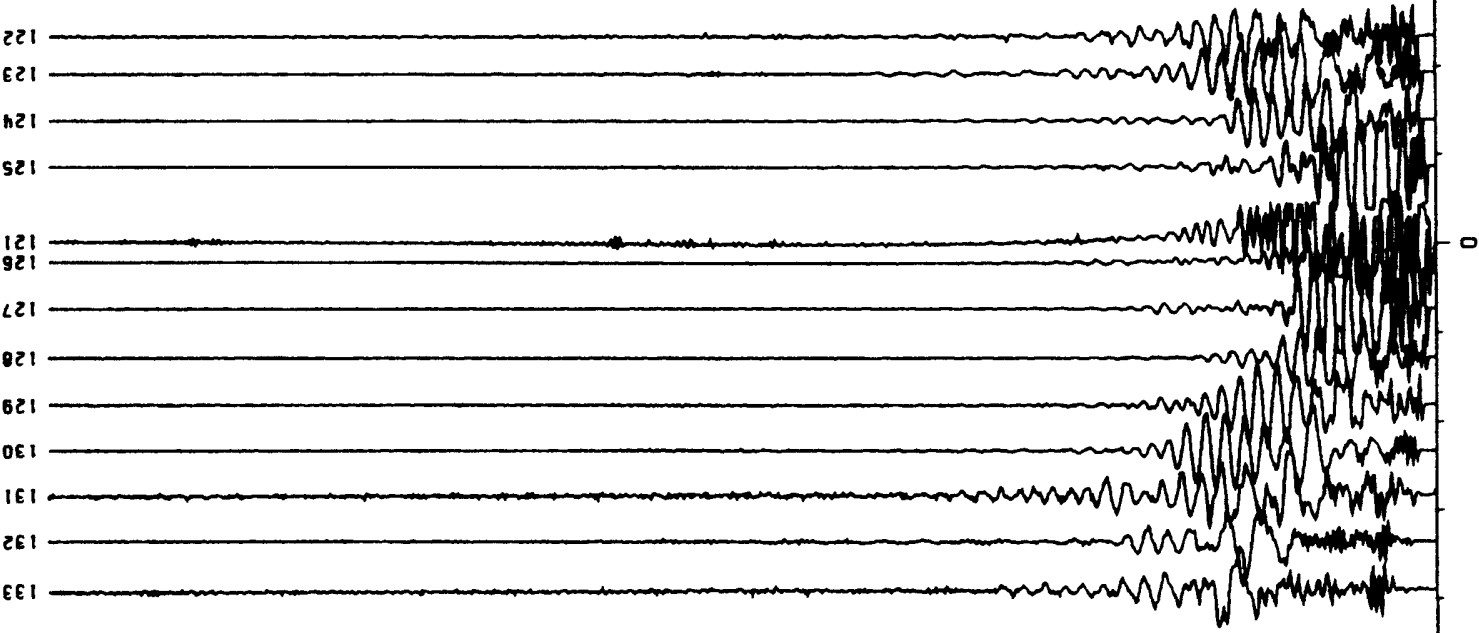
18:

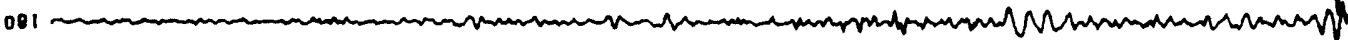
$6\llcorner 1$ QL1 LC1 921 1

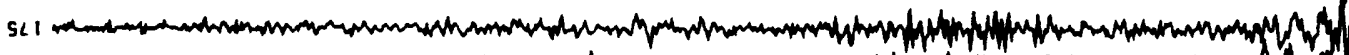

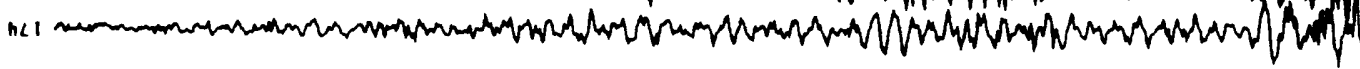

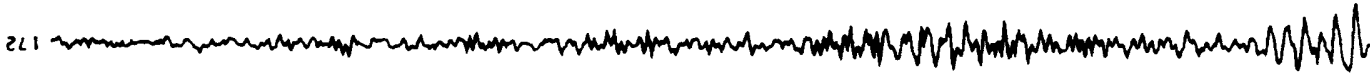

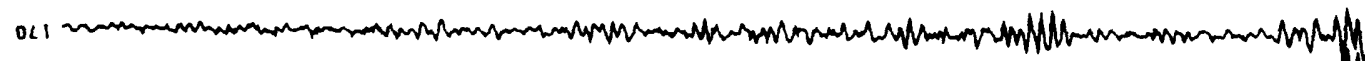
691 2 081 m.nm

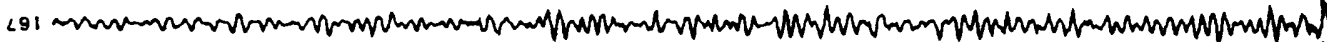

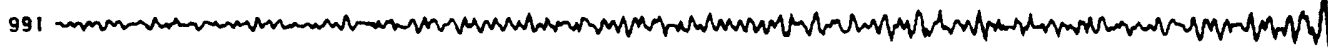
591 m m

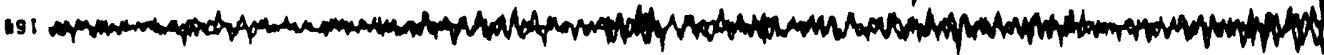
E91

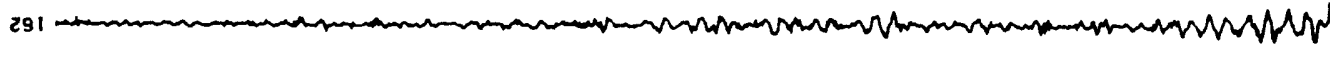
기 


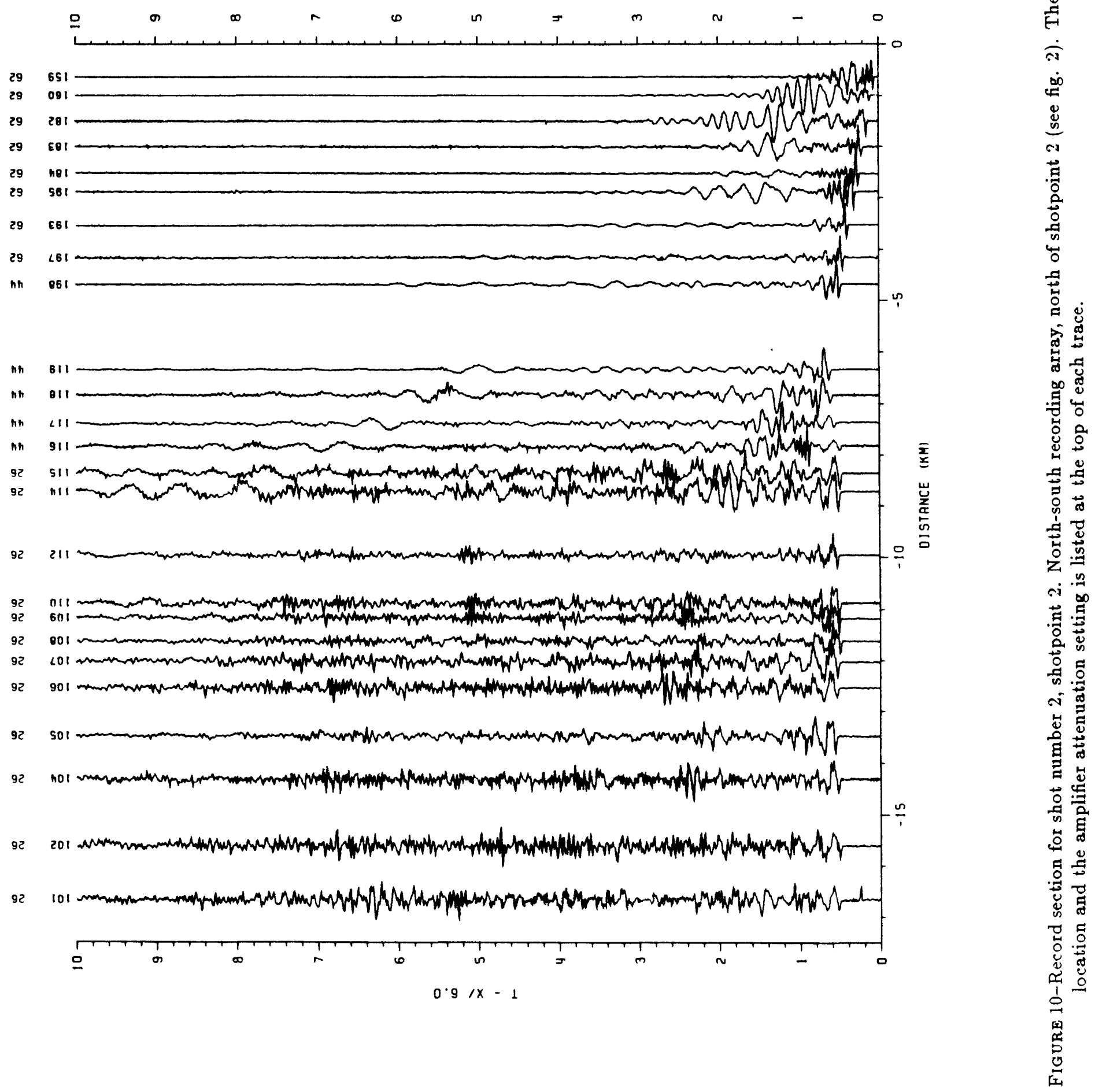




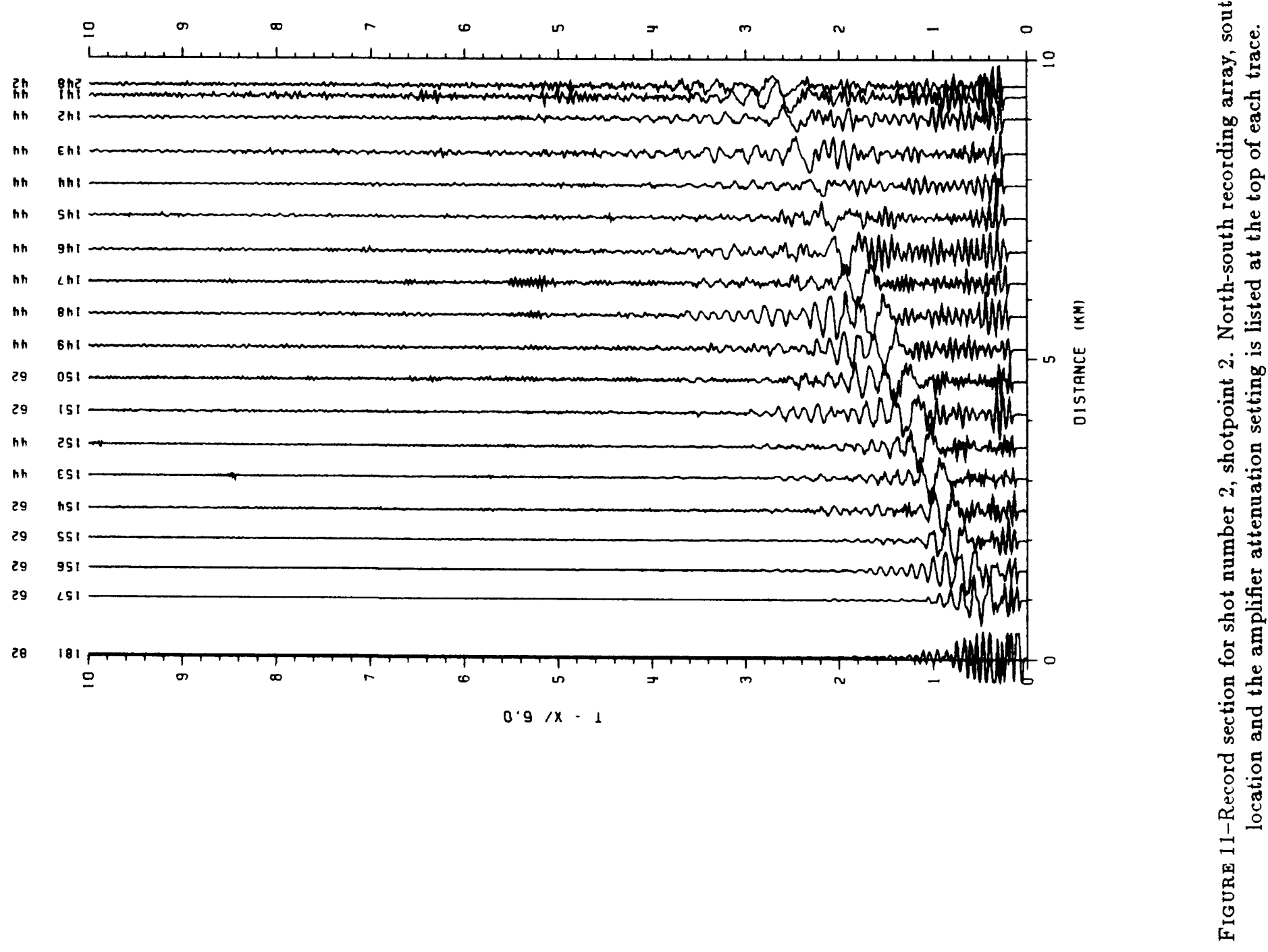


221 - 21 C21 1 21,

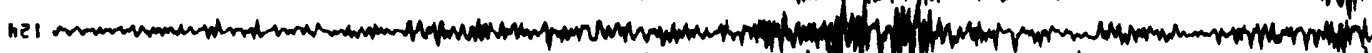

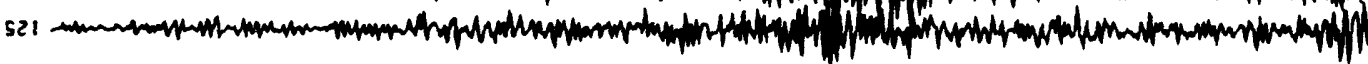
6อ1 L21 021 ב

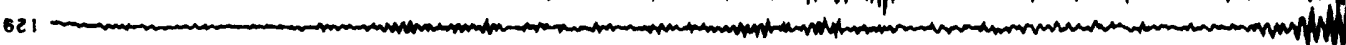
OEI I 1 ב 161 m m ZEI I EE I ב

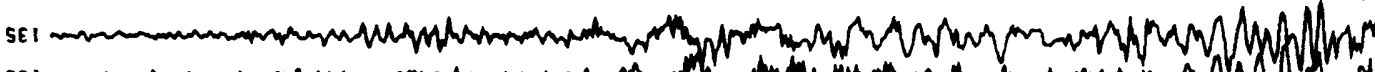

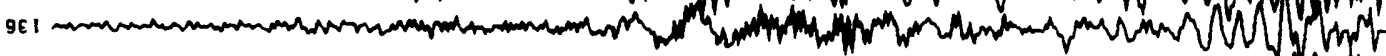

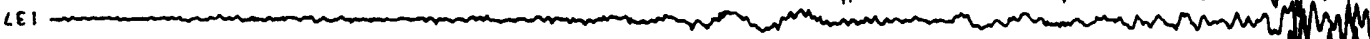
oEl 1 (1) 6E I C81 881 061 281 E61 S61 हo 281 091 181 (4) hLI $\varepsilon \subset 1$ $2 ८ 1$

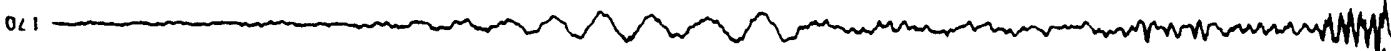
691 1 091 L91 991 (9) ${ }_{191}^{591}$ 191 -

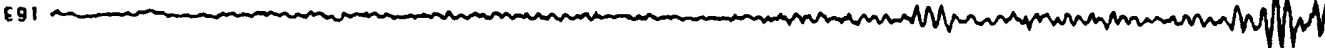
291 on $\mathbf{\infty}$

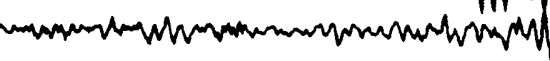




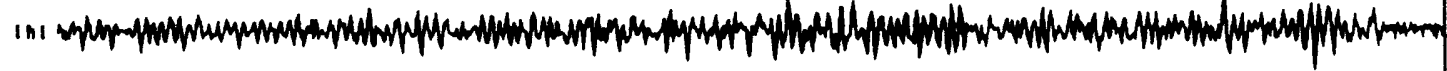

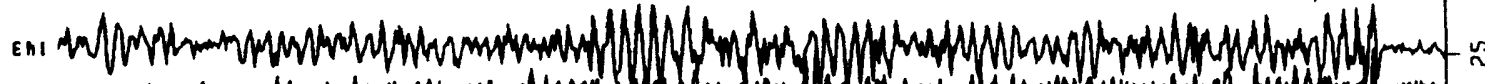

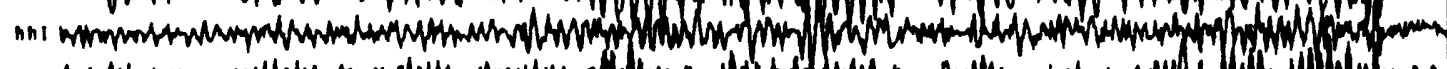

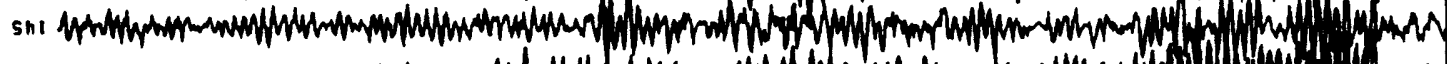

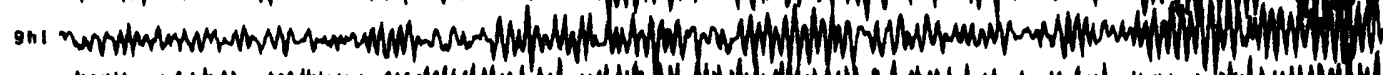

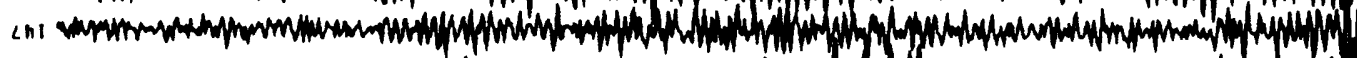

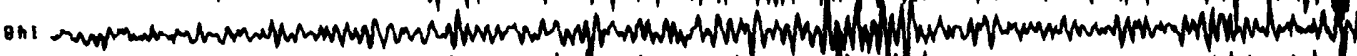

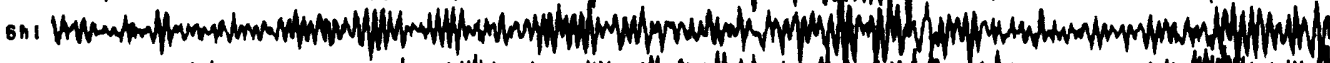

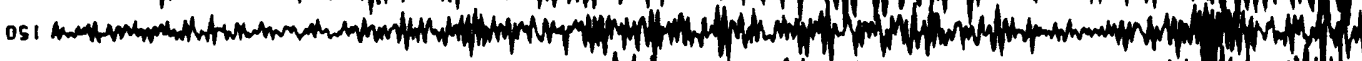

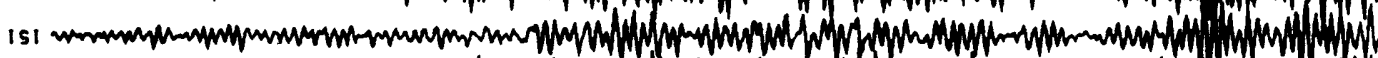

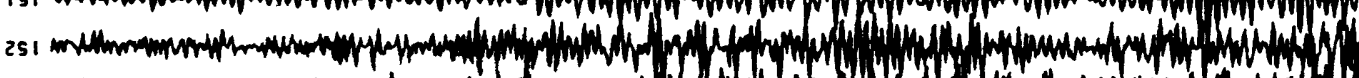

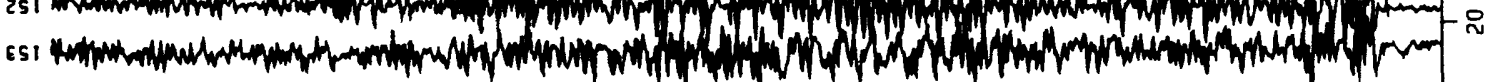

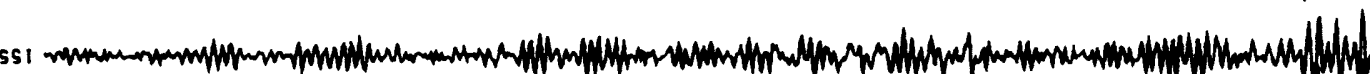

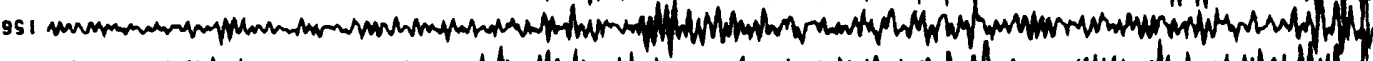

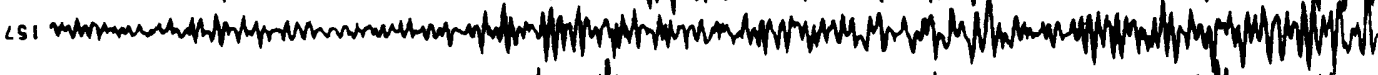

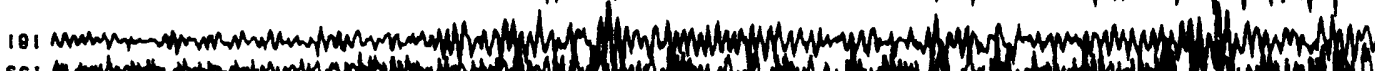

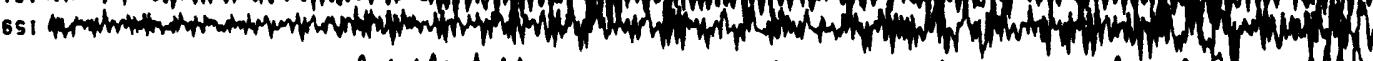

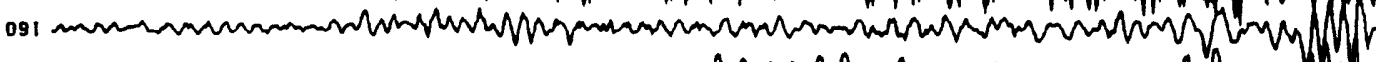

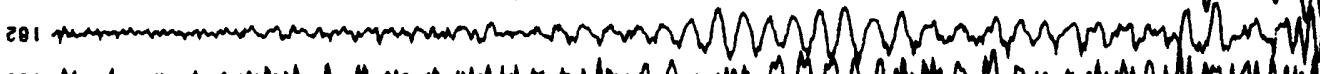

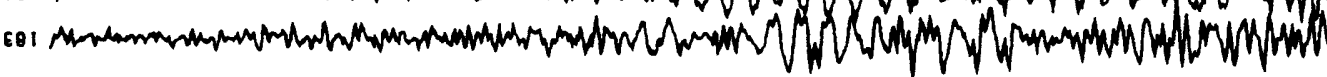

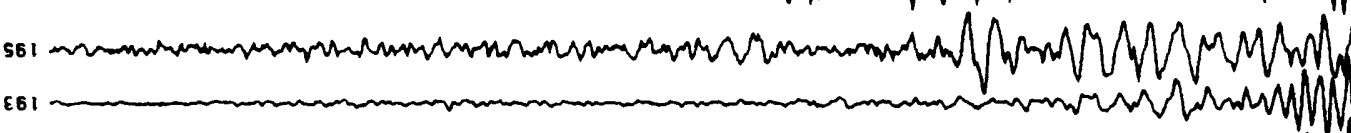
(61 -

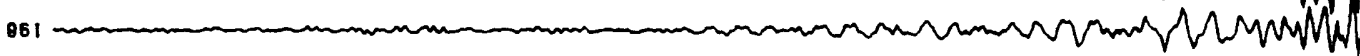

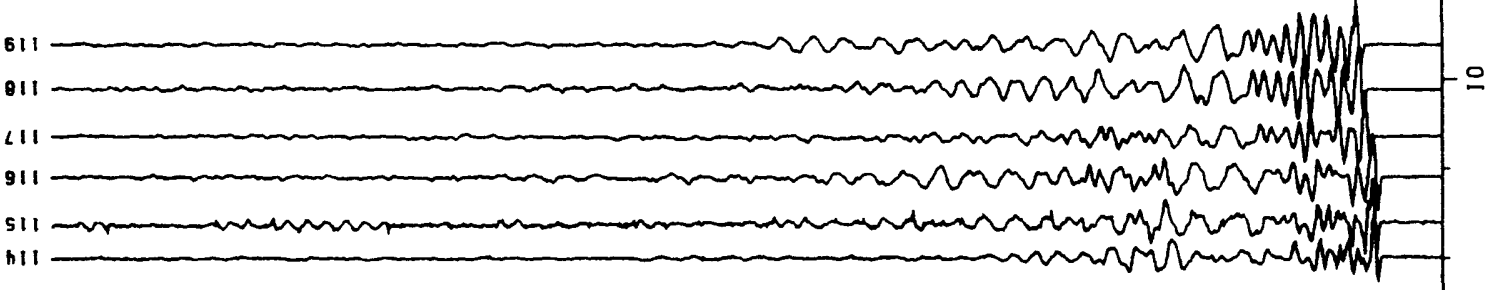

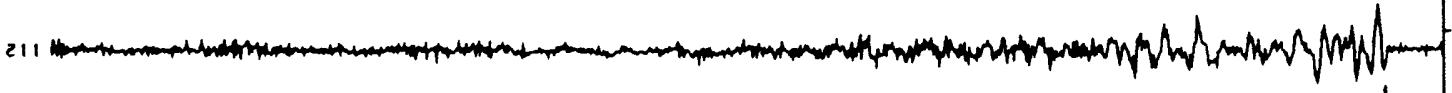
(101) sol רurmonilnt no1

r

0

in 


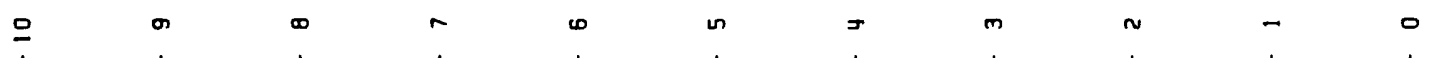

므

o

61

NomuMWh

561

121

$\varepsilon$

281

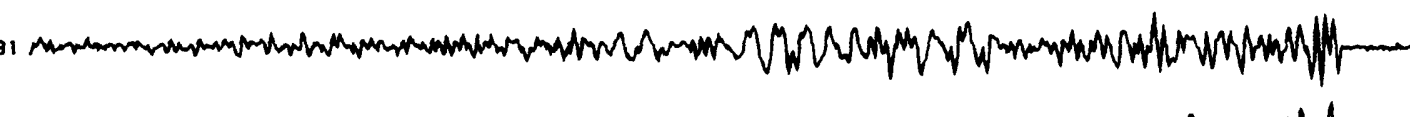

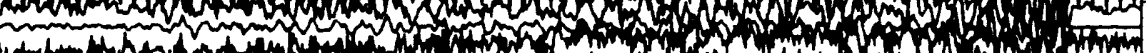
BLI

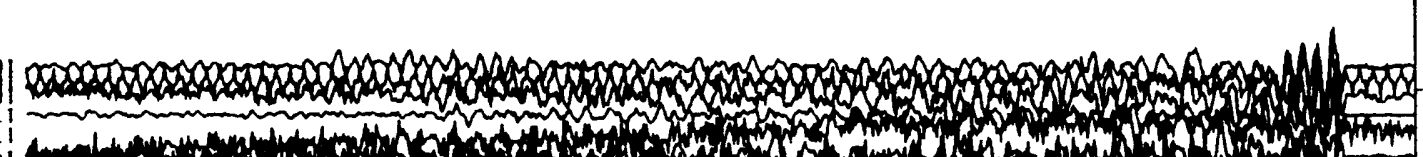
SLI

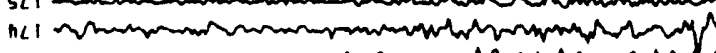

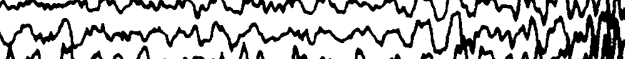

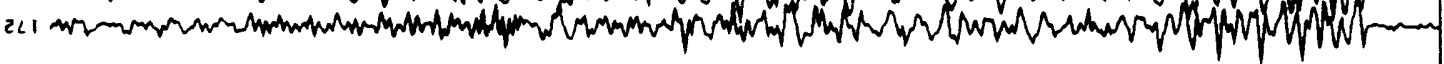

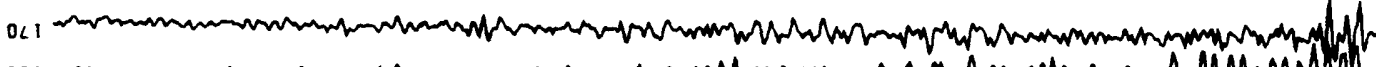

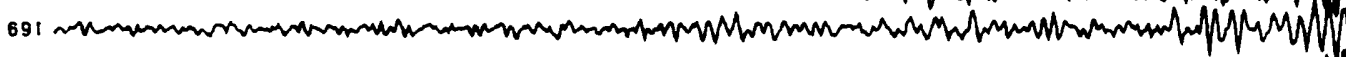

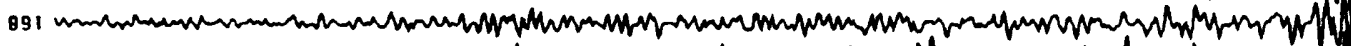

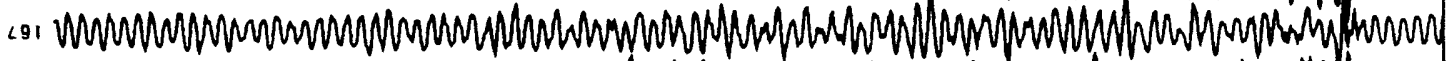
991 -

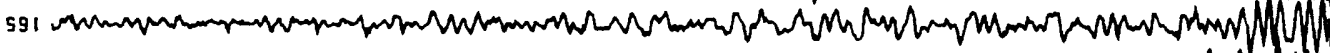

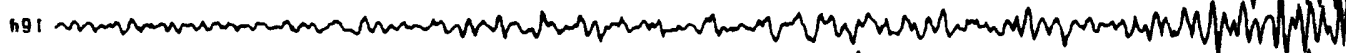

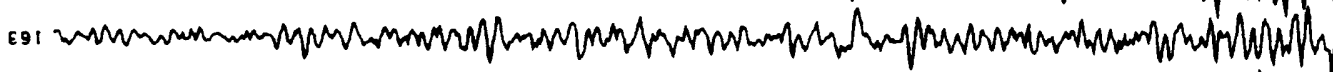

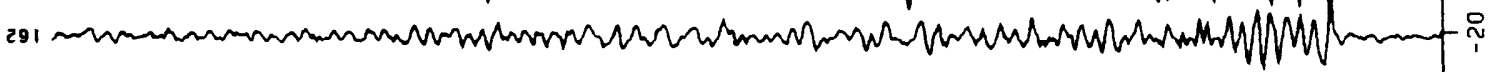


92

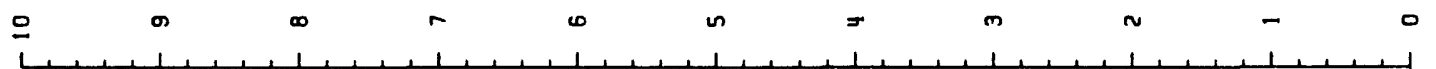

92

221 20.

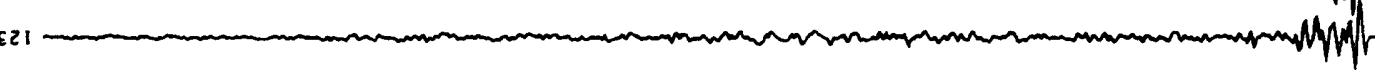

92

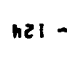

s21

wiply

92

92

92

92

92

92

92

92

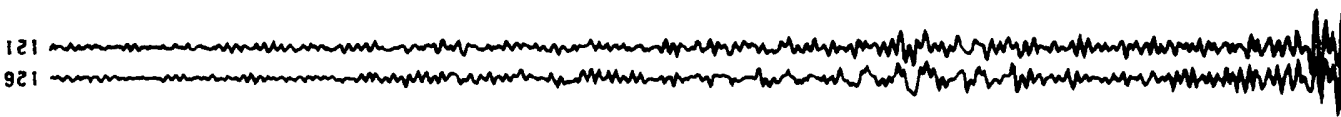

(2)

Mompram

mannd Nun Nom

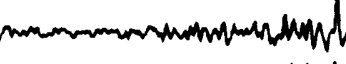

62 I -

0

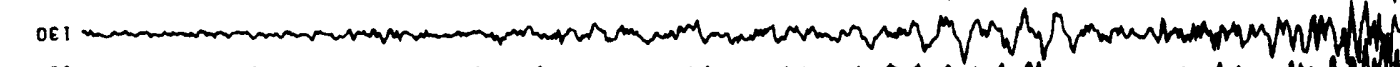

IEI

रE।

$\varepsilon \varepsilon 1$

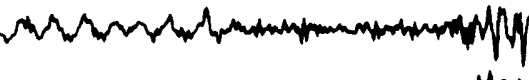
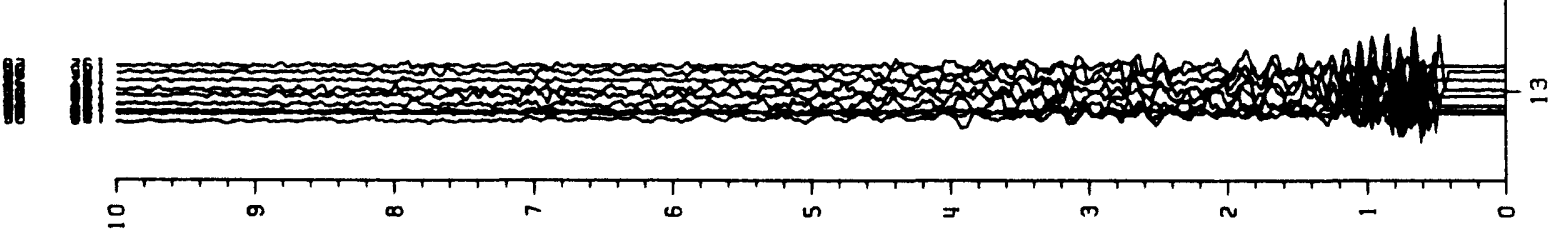

$0.9 / x-1$ 


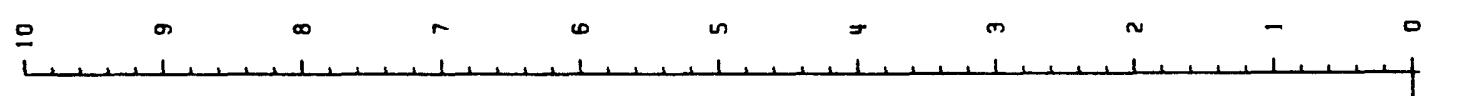

hh

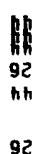

101

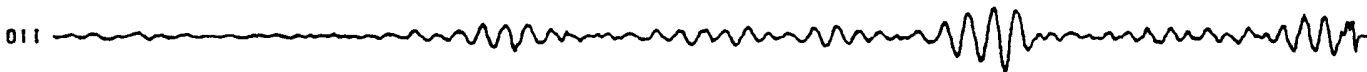

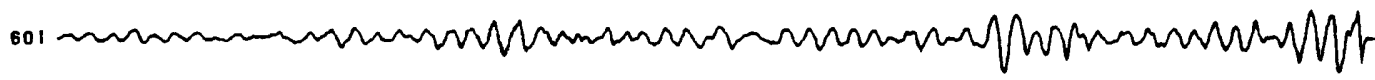

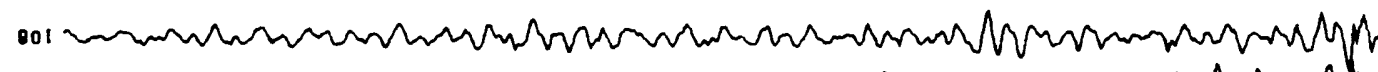

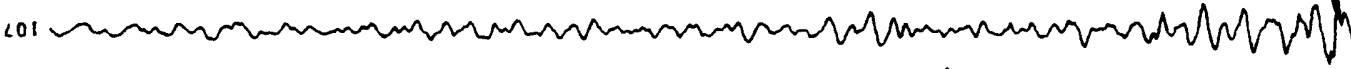
901 M sol $\widetilde{1}$ nol

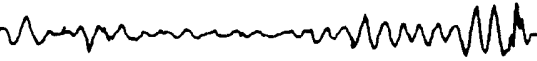

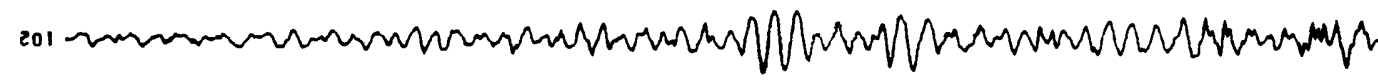

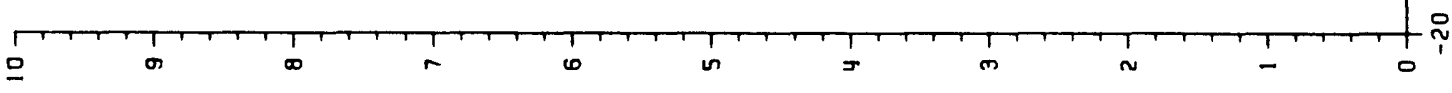
$0.9 / x-1$ 


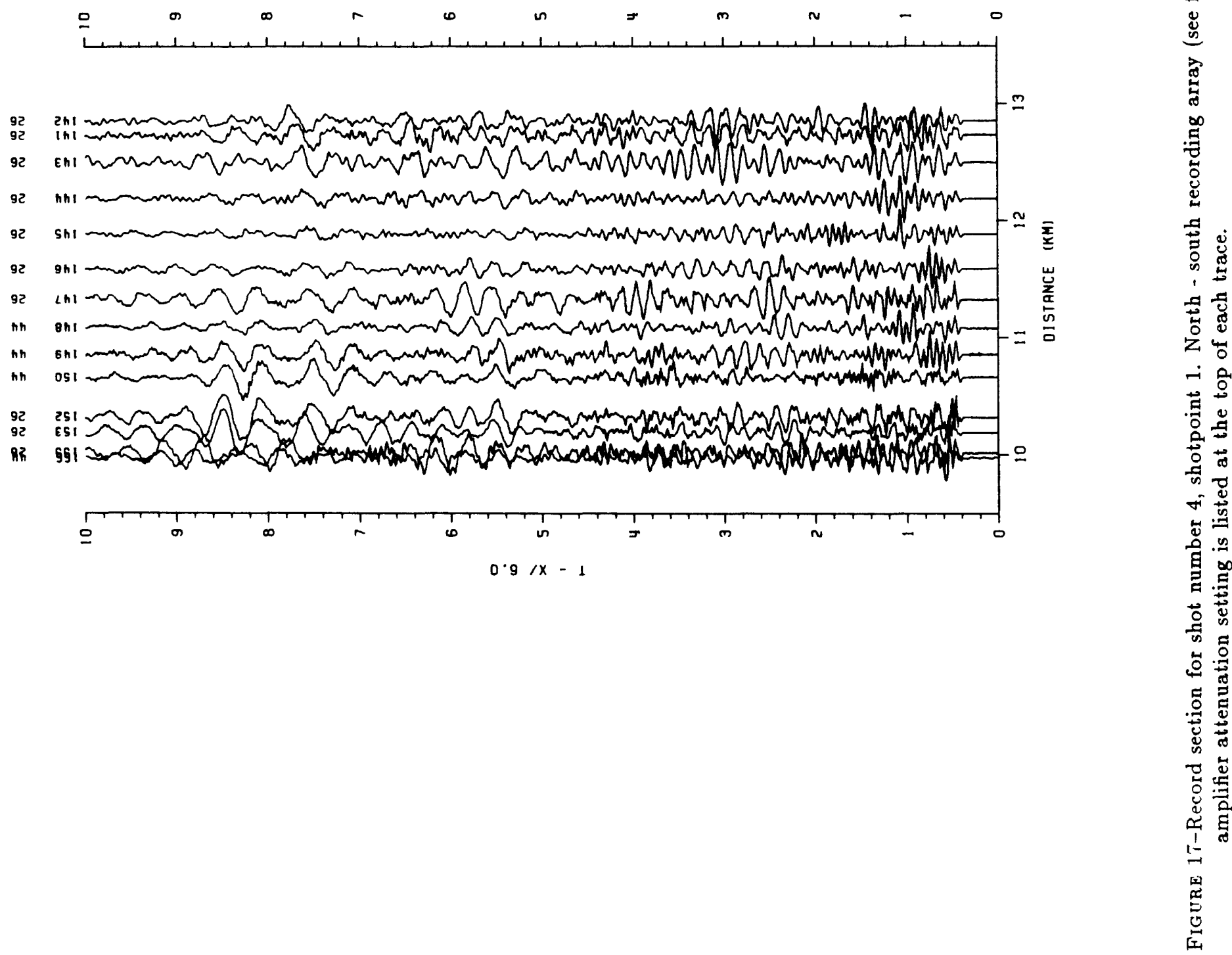




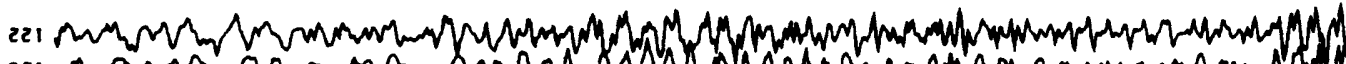
E21 vornon

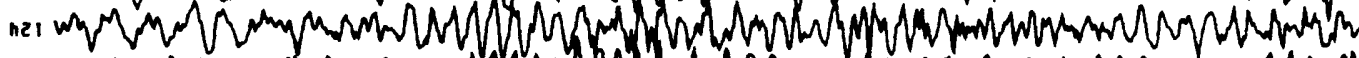

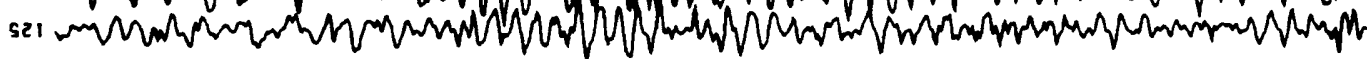

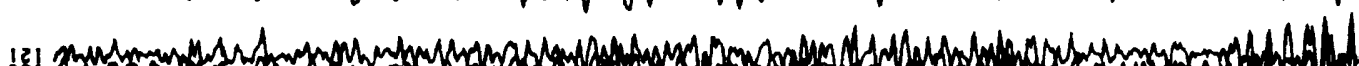

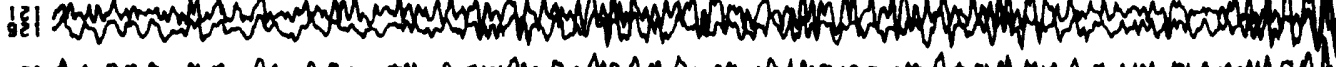

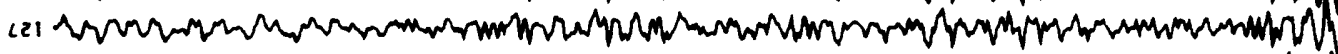

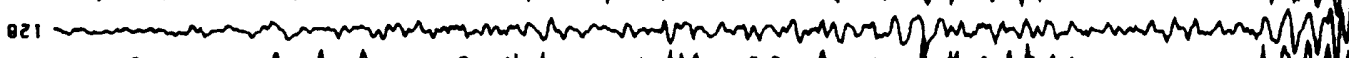

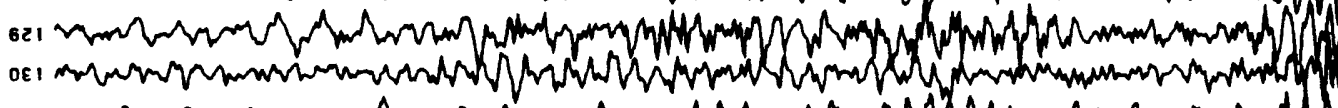

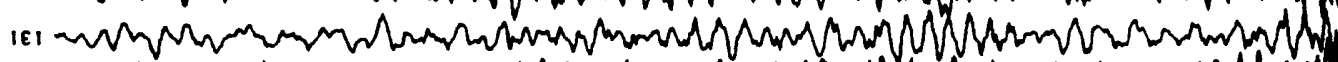

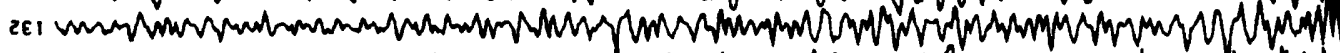

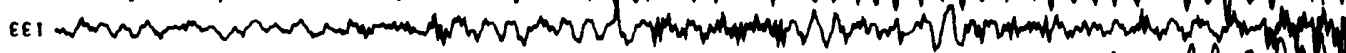

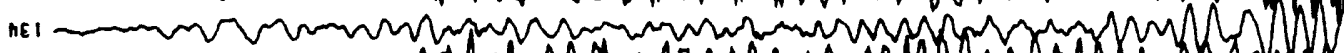

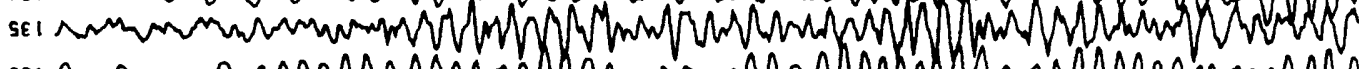

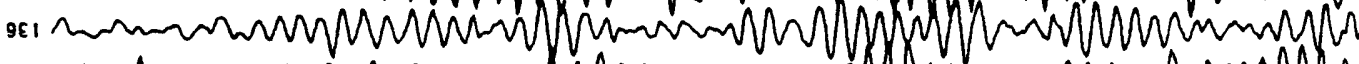

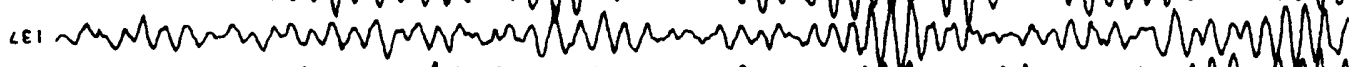
Be1 (2) :E1 co1 B81 Consm $061+2$ $161 \mathrm{r}(\mathrm{C})$ ${ }_{261} \sim \mathrm{Cr} N \mathrm{~N}$ :61 - (n)

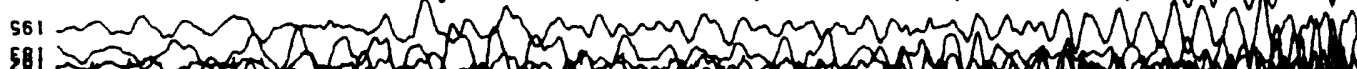

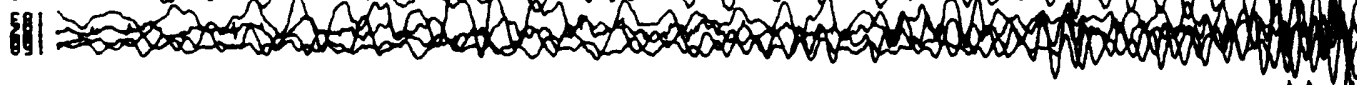
(1) 

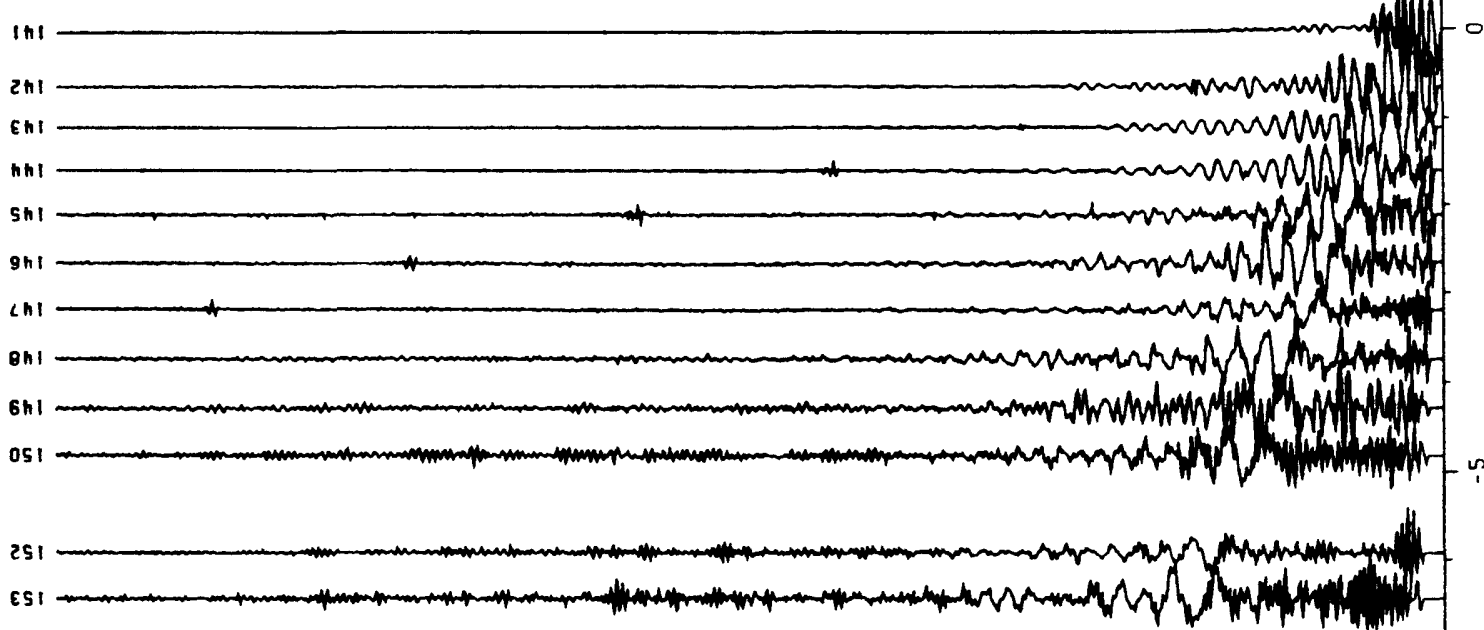

SS1 -

9si 1 -

LS1

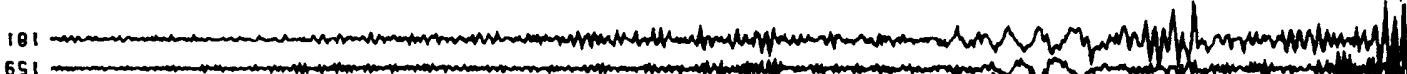

091 (1)

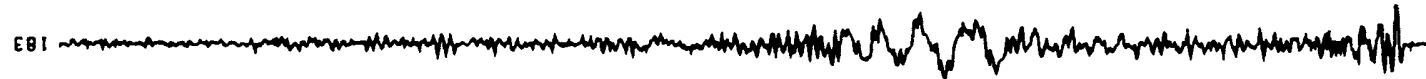
561 -

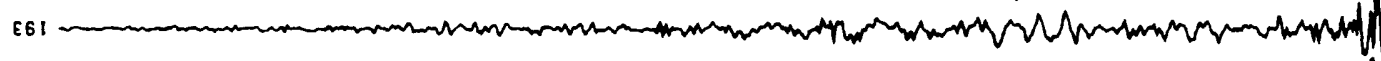

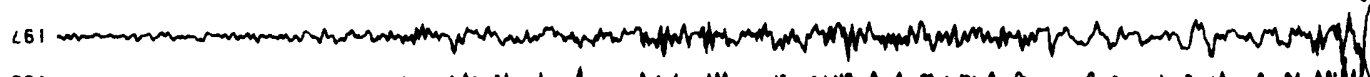

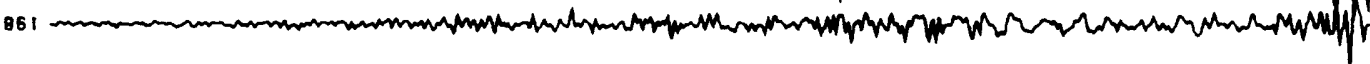

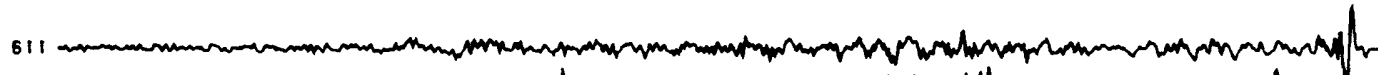

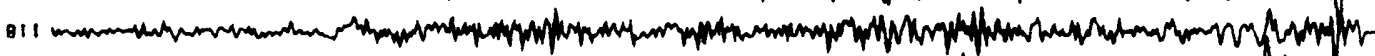

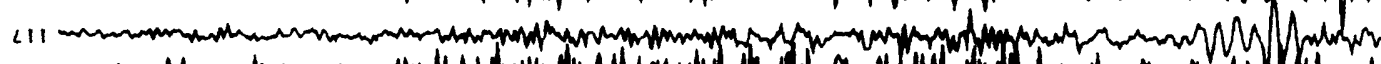

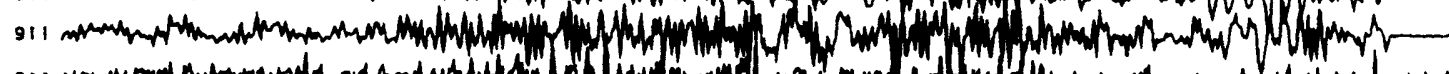

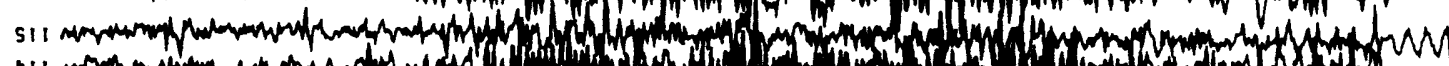

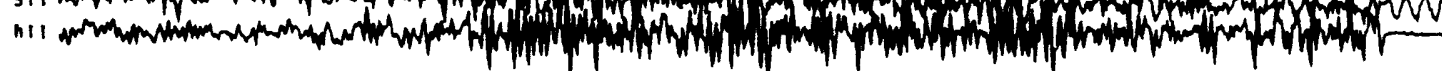

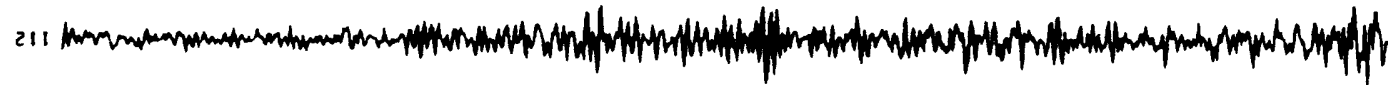

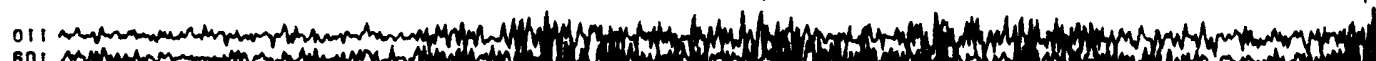

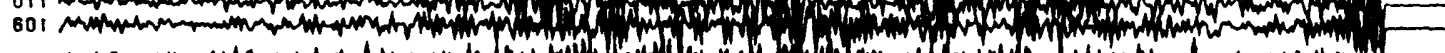

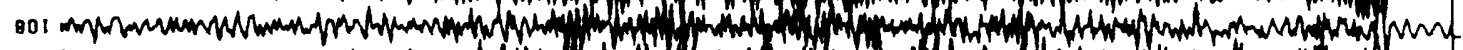

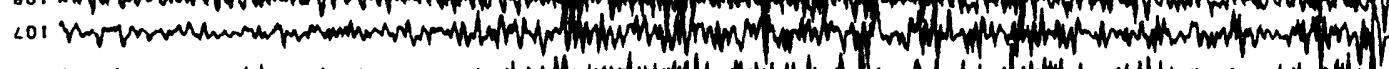

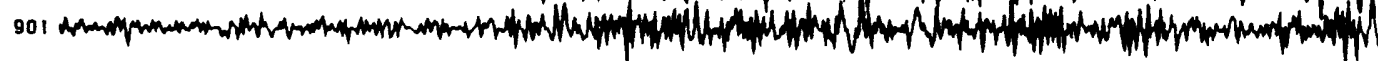
SO1 m

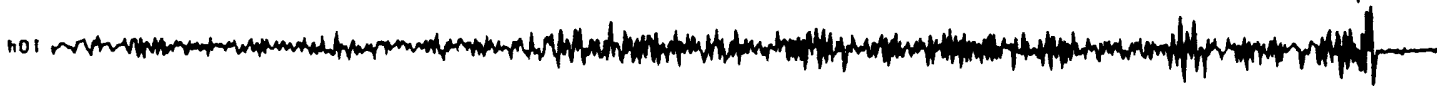

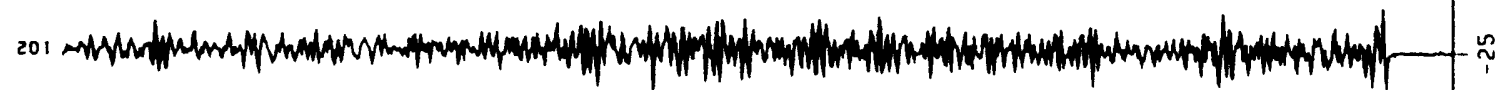

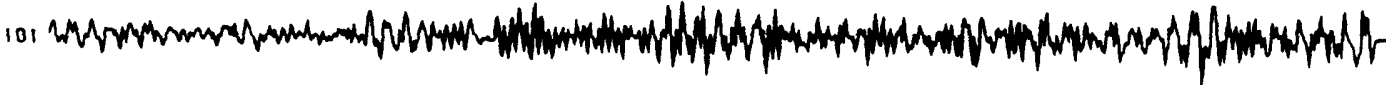

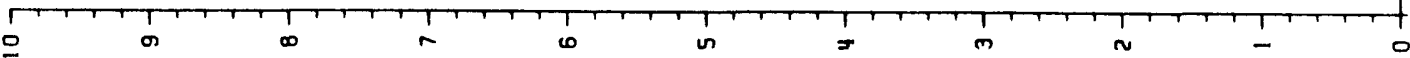




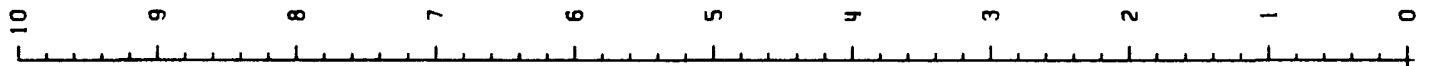

181

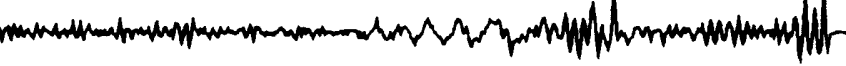

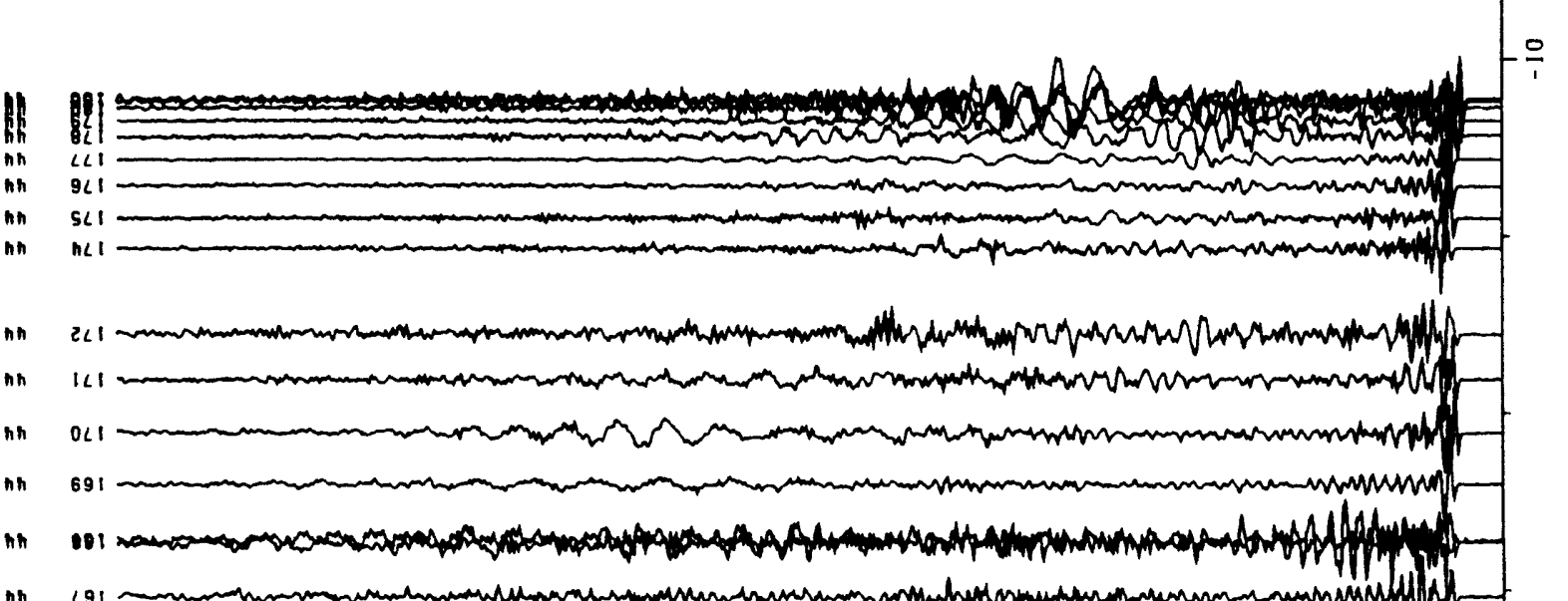

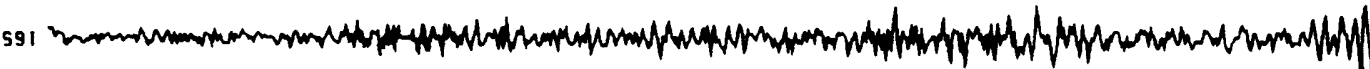
म91 sestepentive

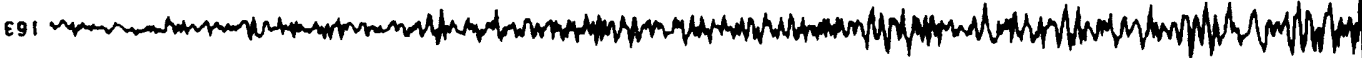

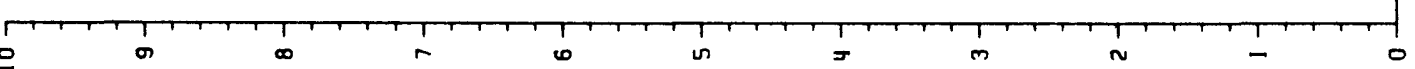

$0.9 / x-1$ 


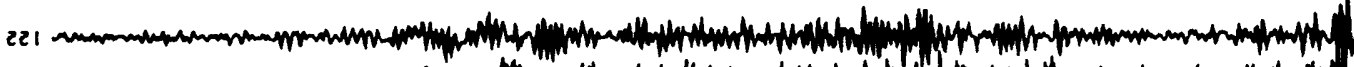

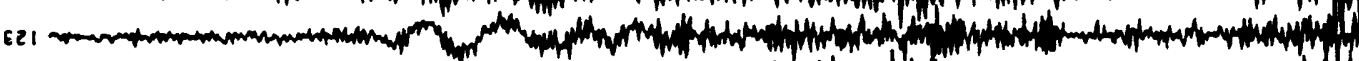

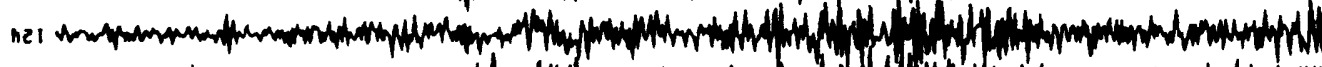

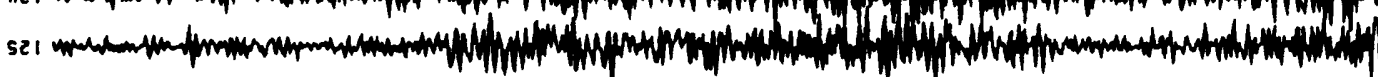
(2)

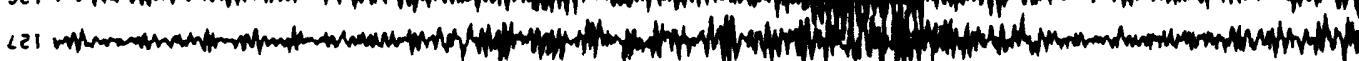
021 621 oE

IEI

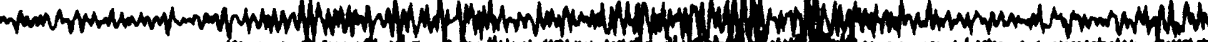

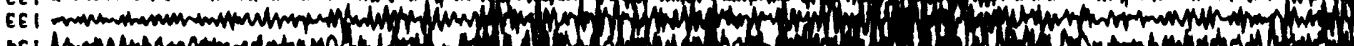

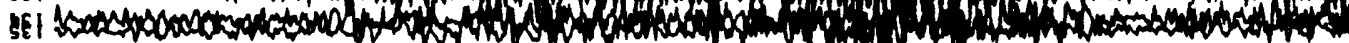

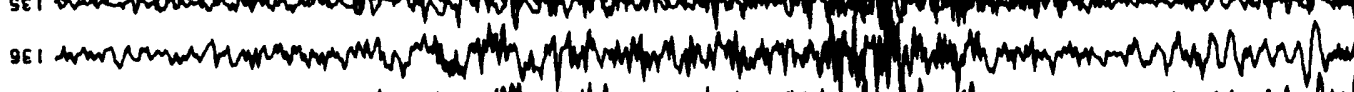

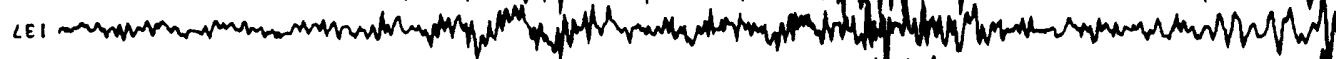
OEI I

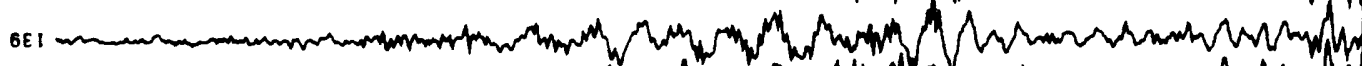

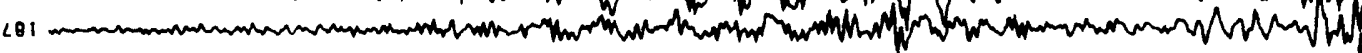
891

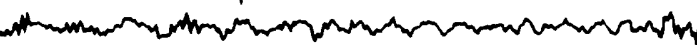

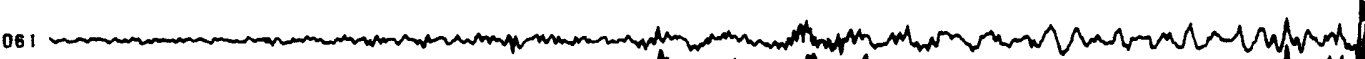
161 m 261 (1)

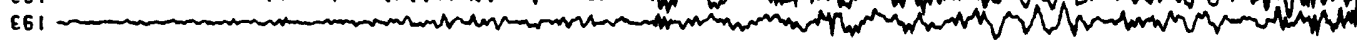

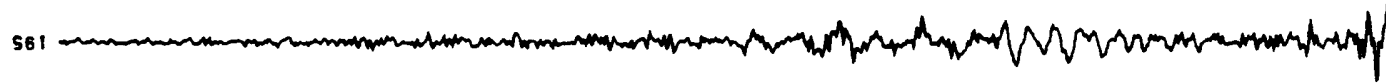

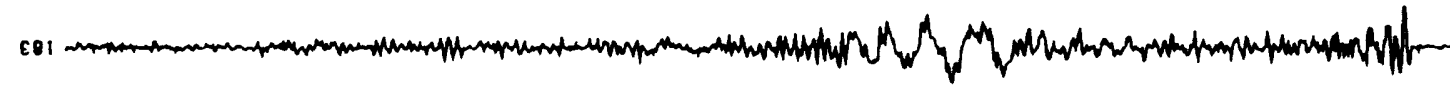

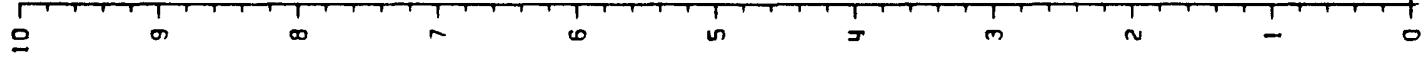

$0.9 / x-1$ 


$$
\text { a }
$$

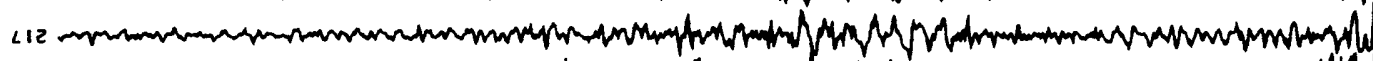

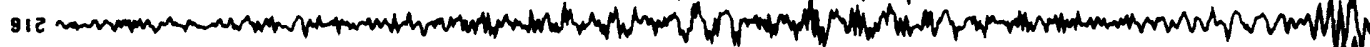
512 -

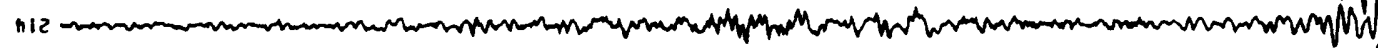

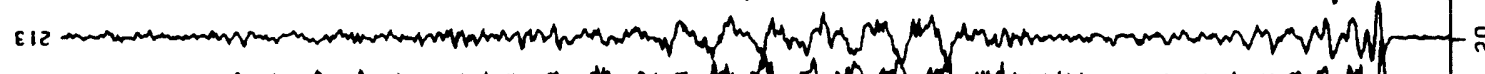

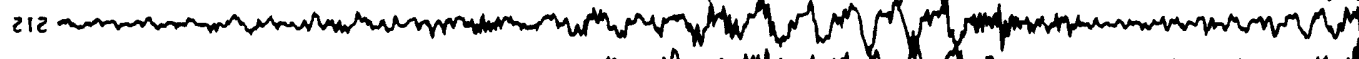

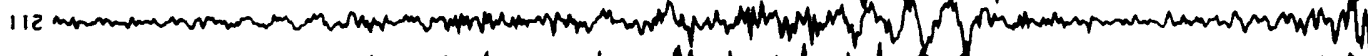

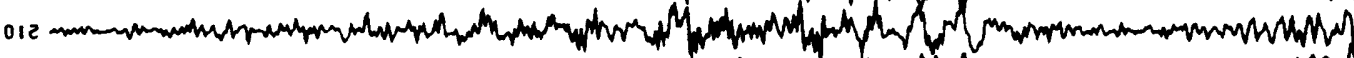

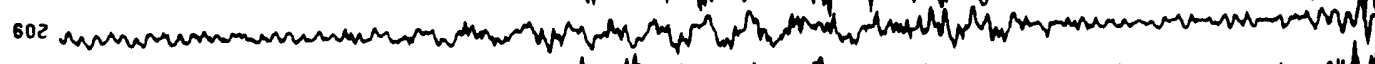

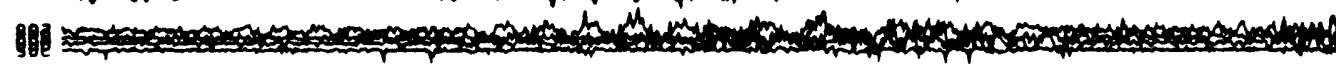
02 O2 202

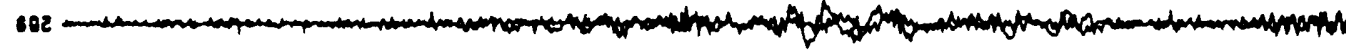

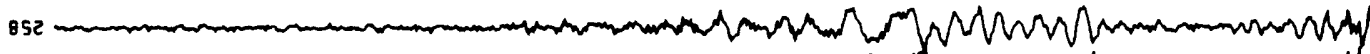
152 -

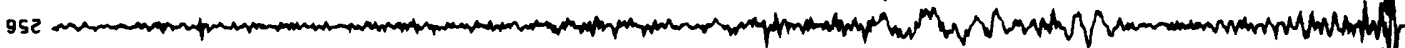
ss2 -

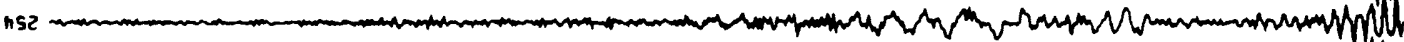

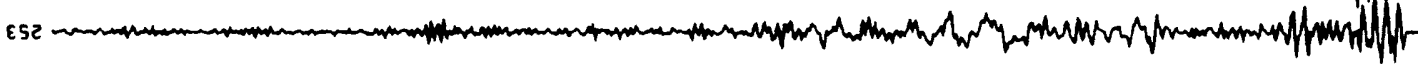
Sh2

$\infty$ $0.9 / x-1$ 


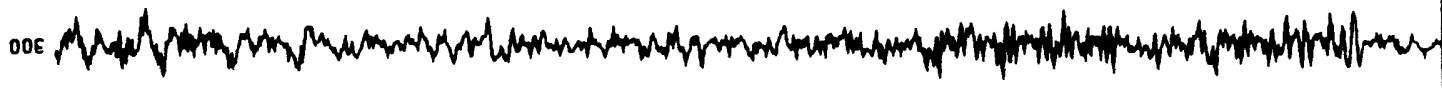

262 m-10

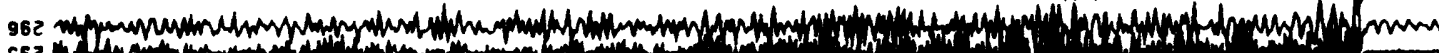
C6z

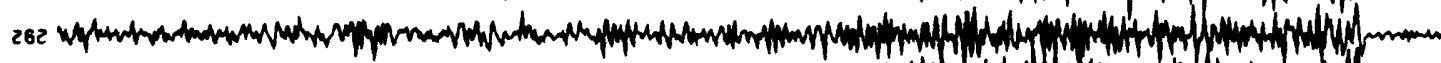

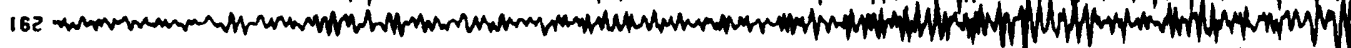
062 r

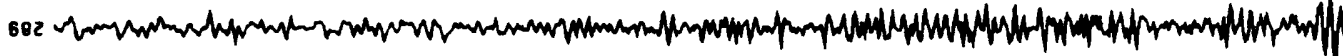
ioz

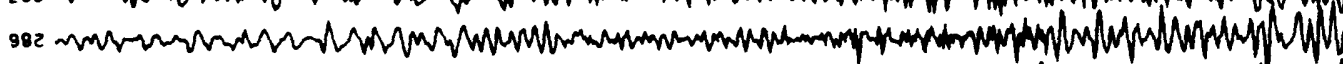

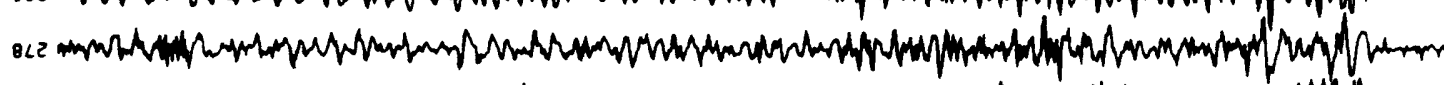

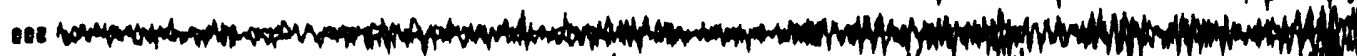
3 践 sez

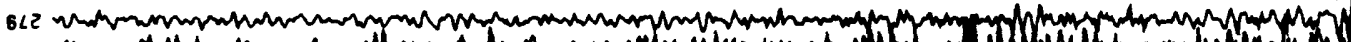

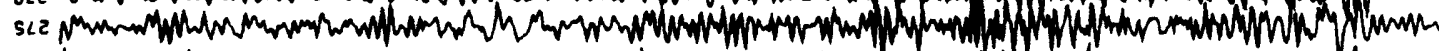

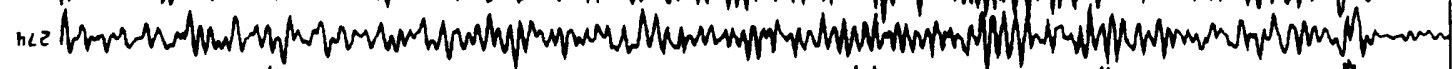
SEe tor

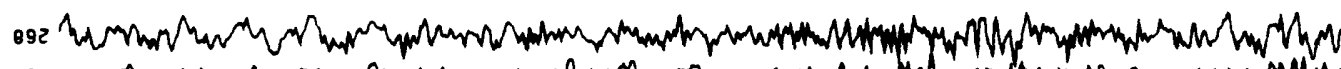

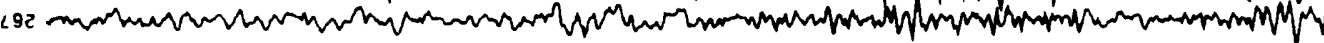

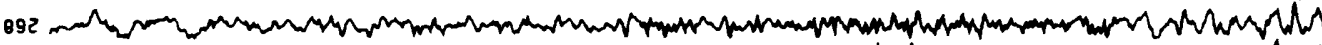
592 nuthmon 192 2010 E92 (1) 192 2010 2E2 2 2

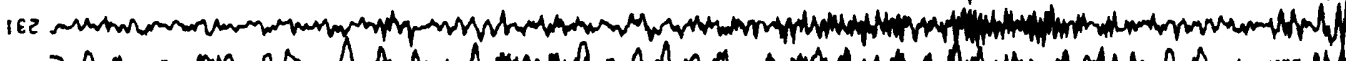

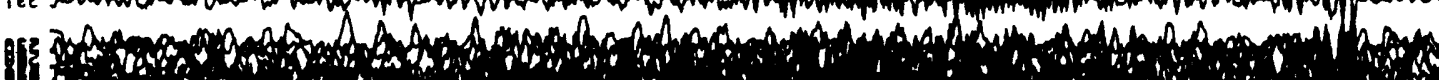

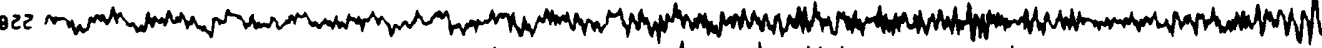

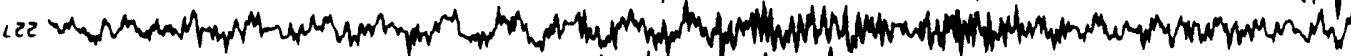

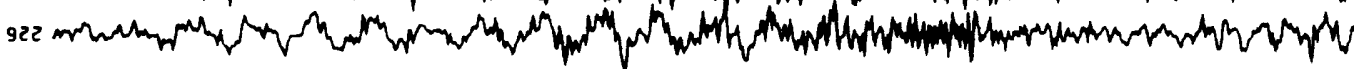

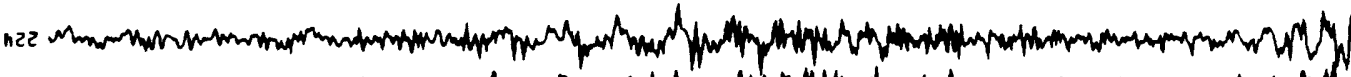

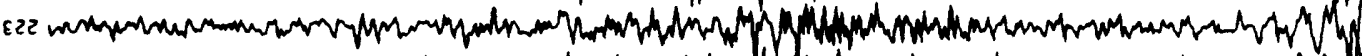

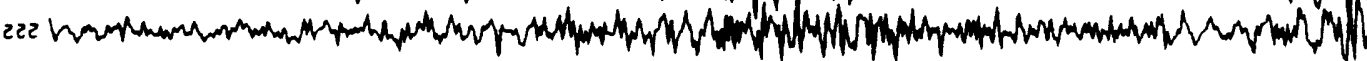

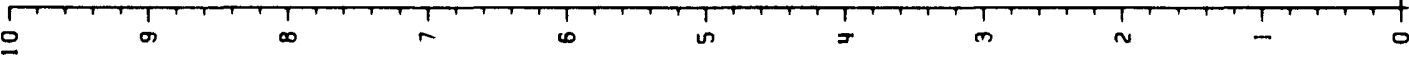




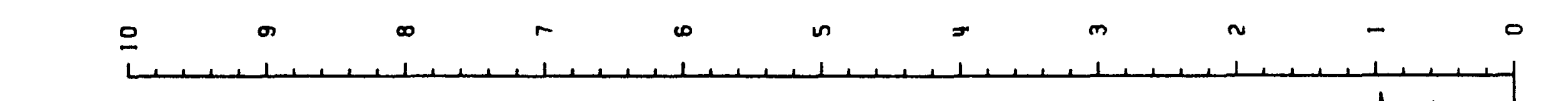

2h

2h

2h

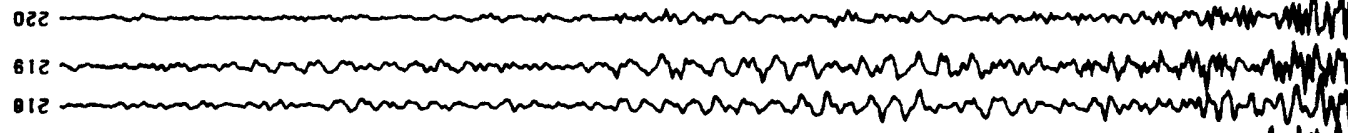

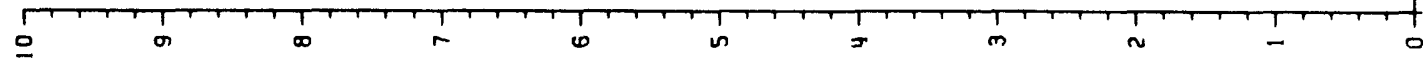




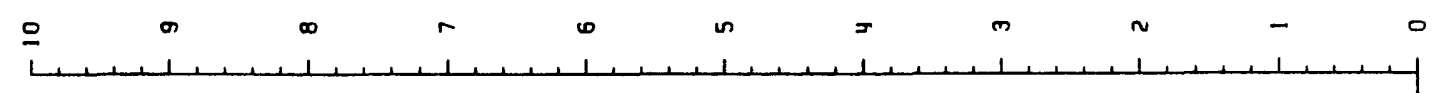

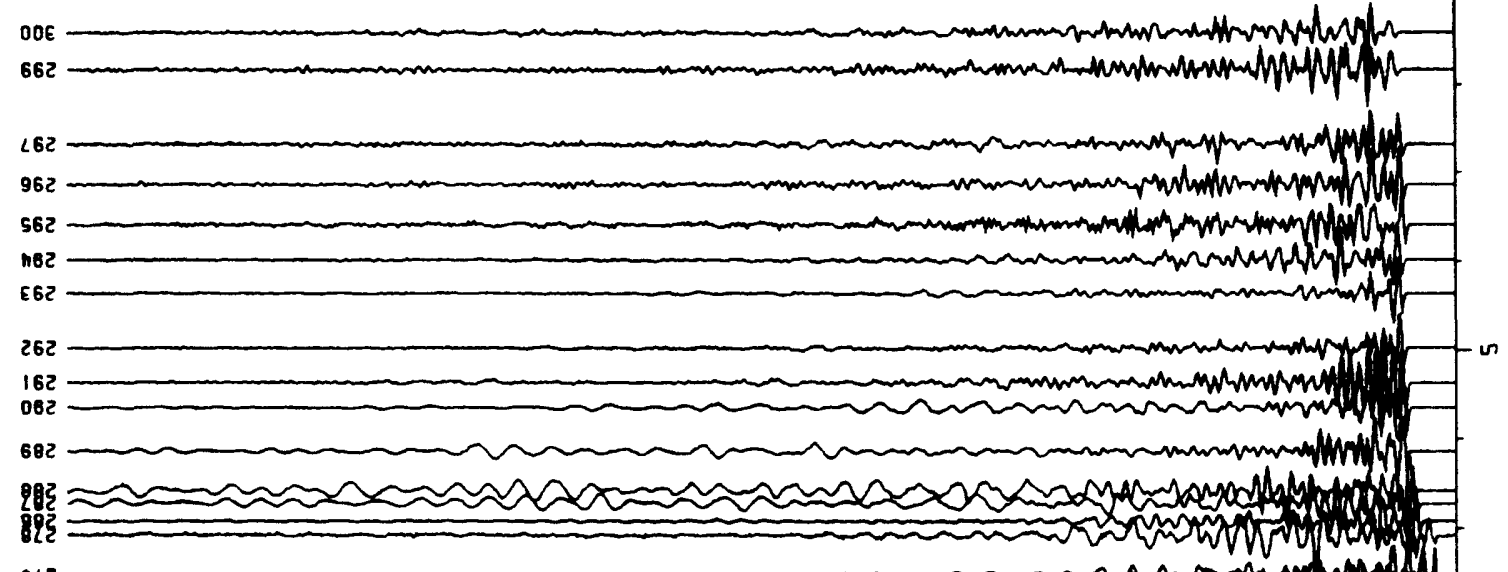
$692 \sim 20$

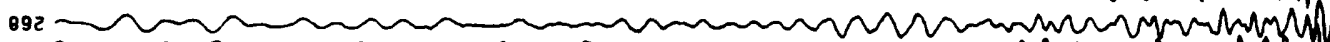
$292 \sim 2 c$ $992 \sim$ (92)

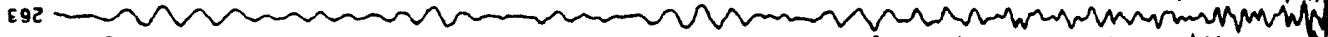
192 (n)

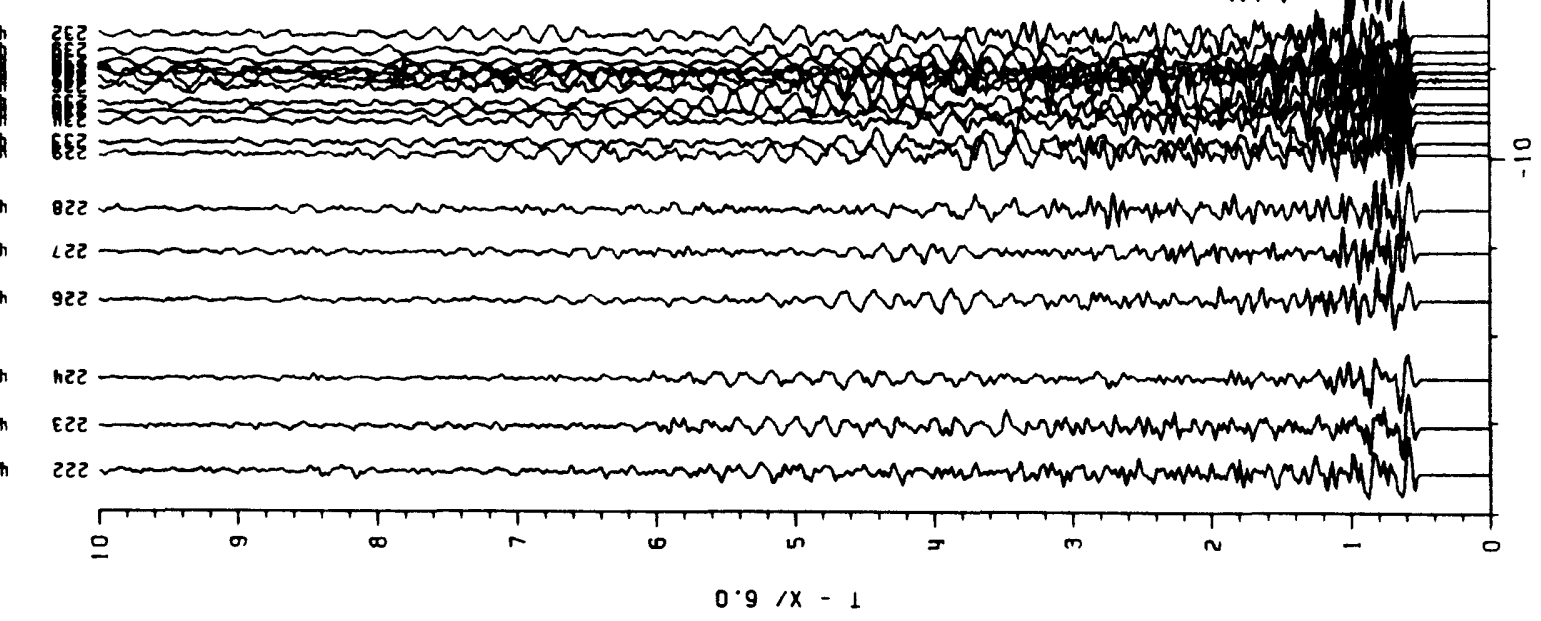



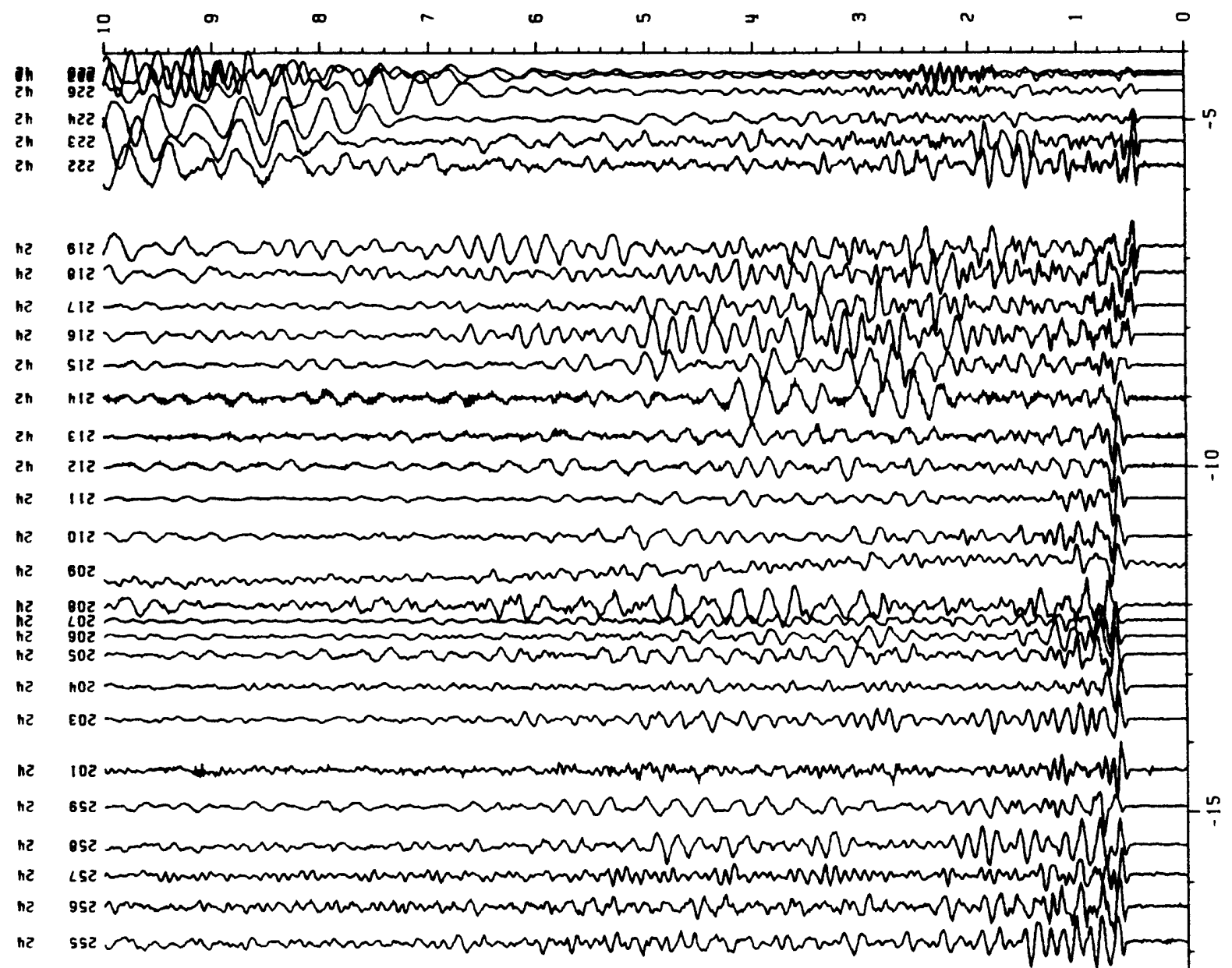

csi am-1)

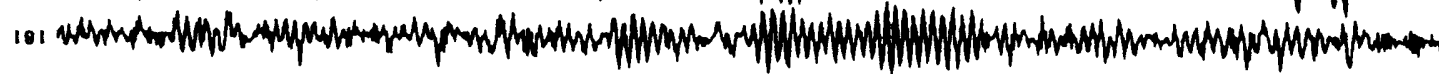

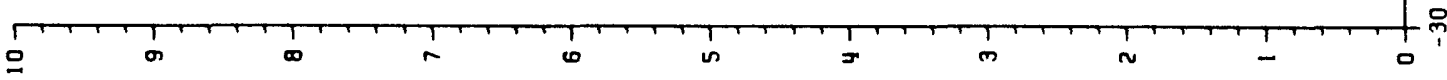




$$
\text { 음 }
$$




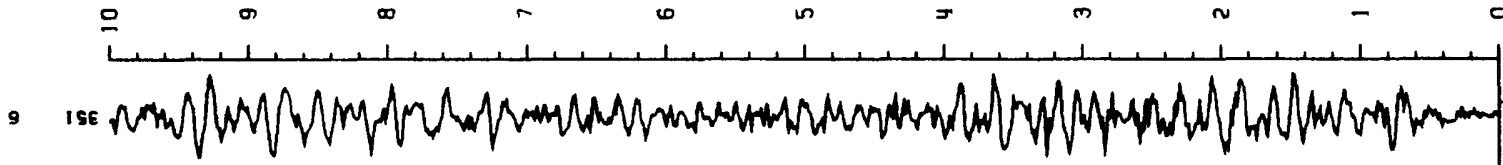

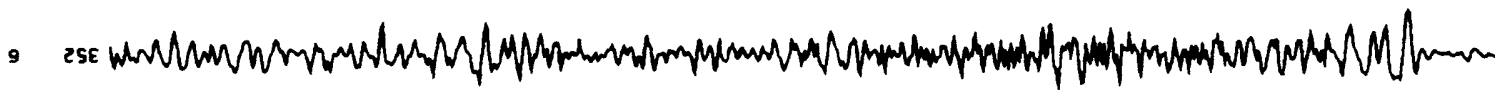

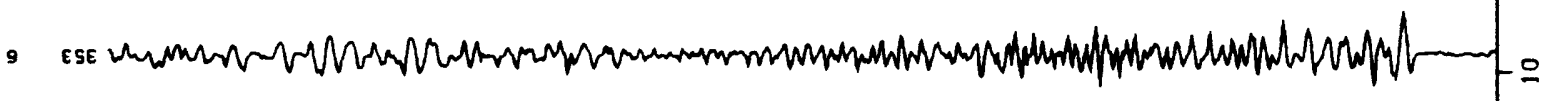

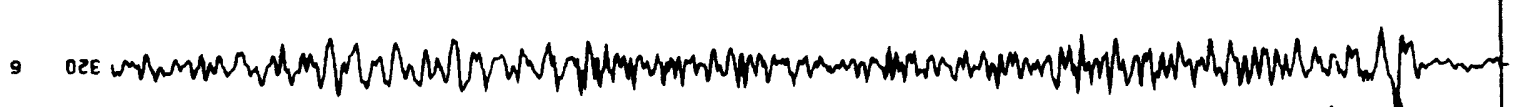

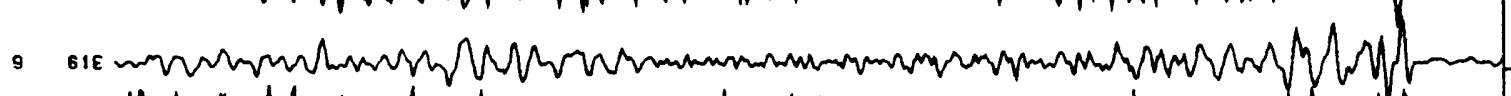

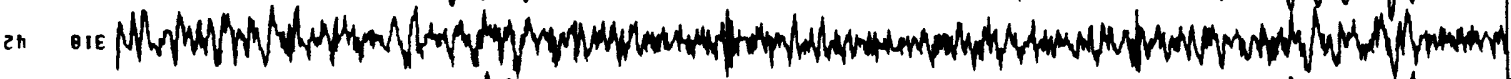

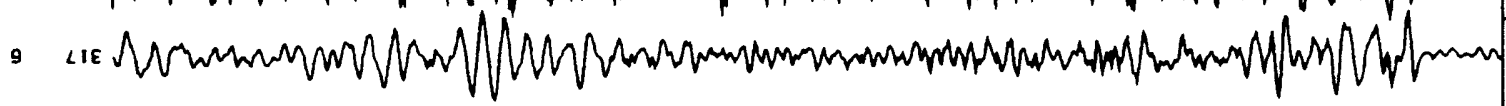

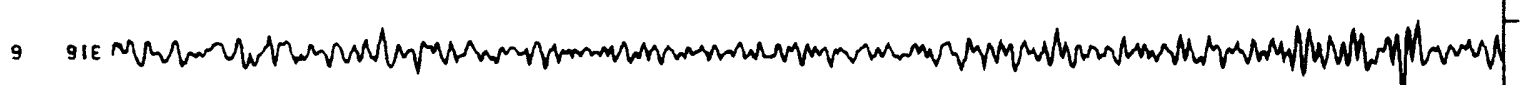

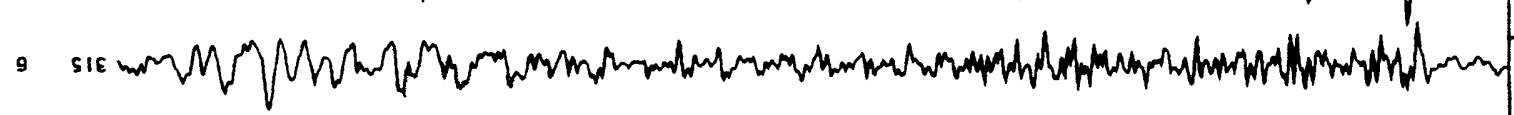

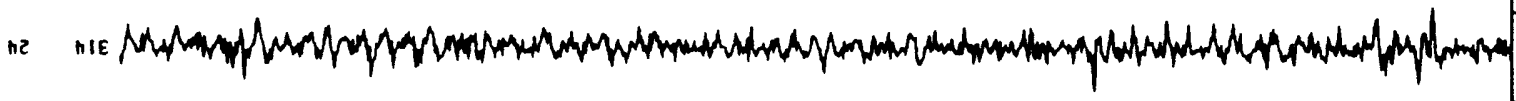

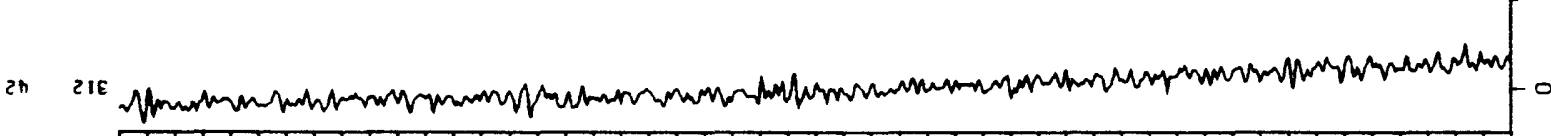

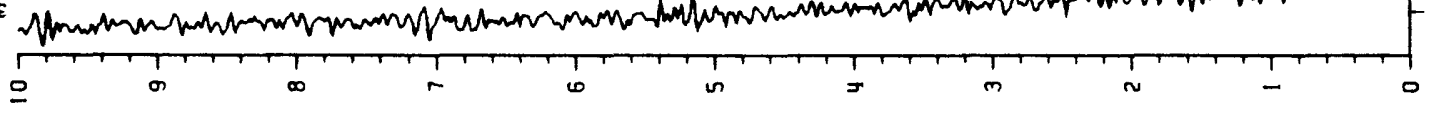

$0.9 / x-1$ 
a

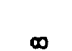

IIE
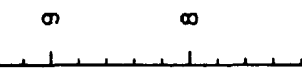

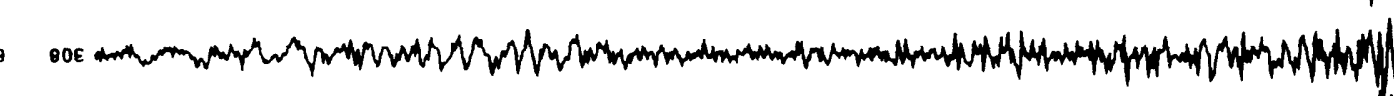

9

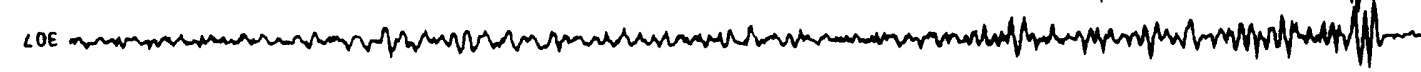

(5)

$$
\text { , }
$$

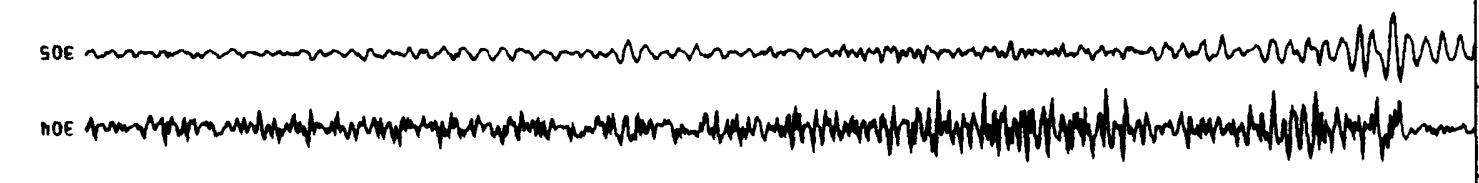

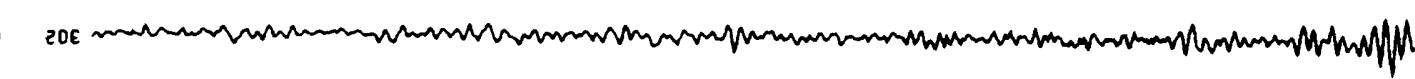

he 


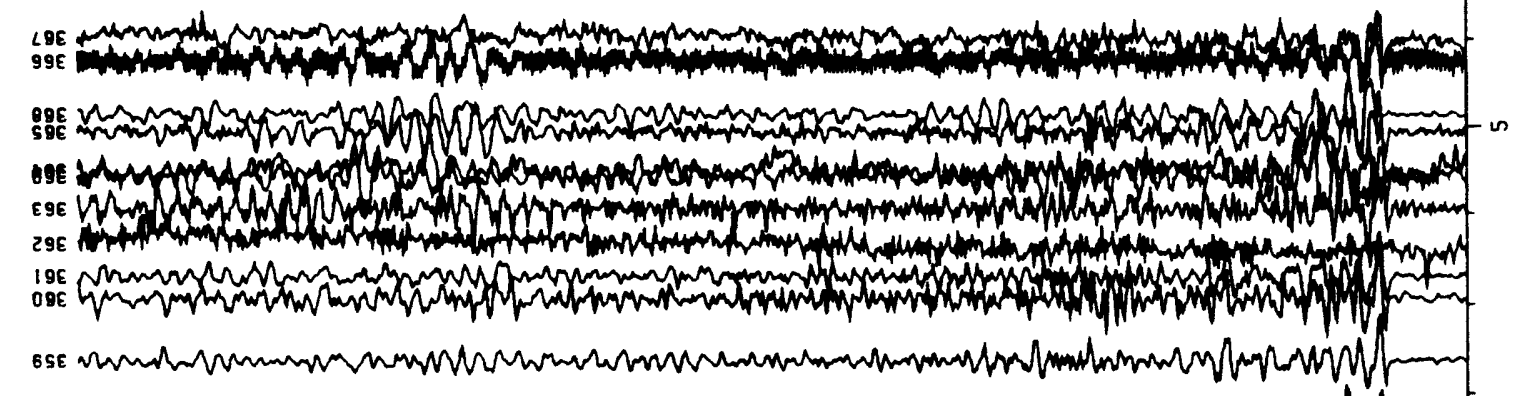

8.

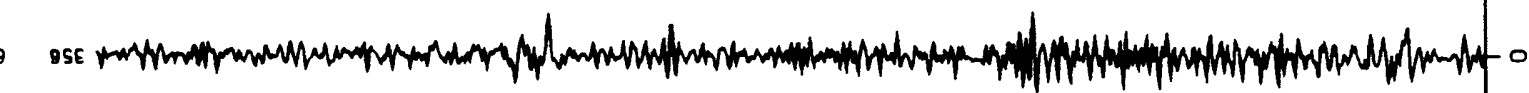

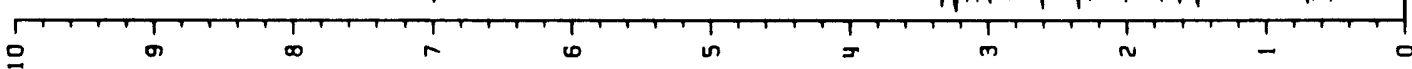


음 o $\infty$ $r$ $\omega$ n m $\circ$

9 eе

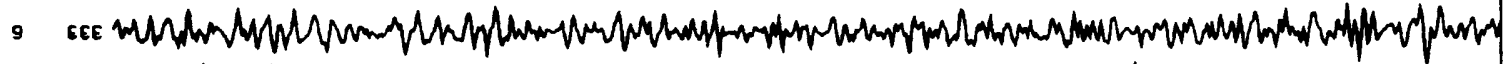

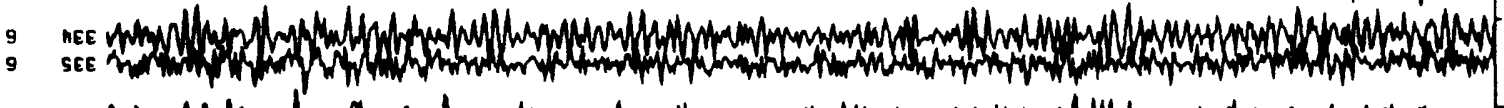

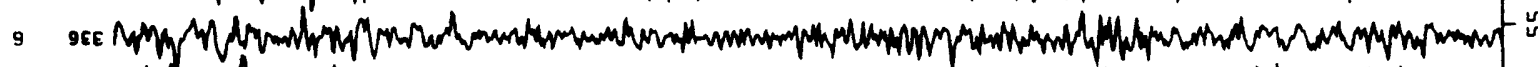

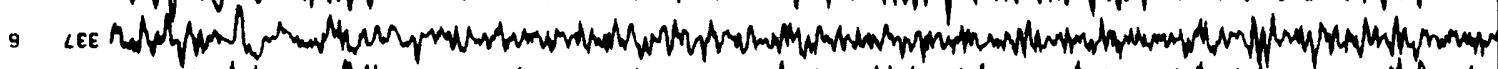

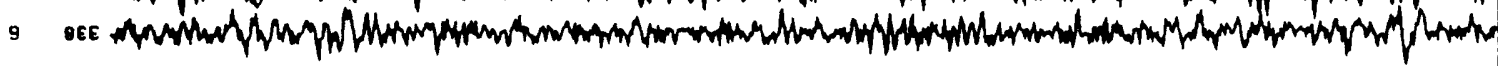

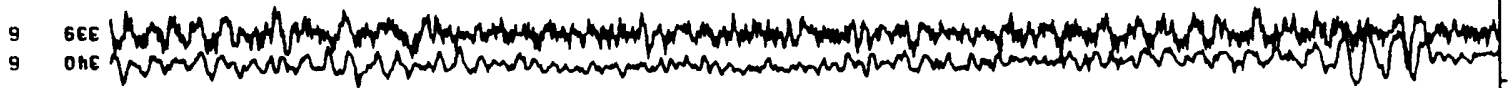

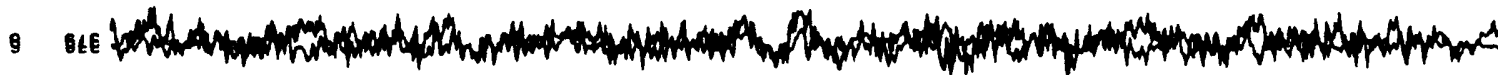

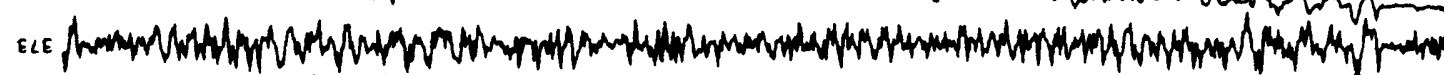

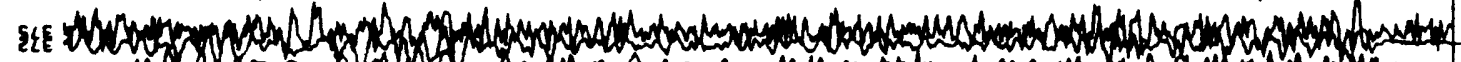

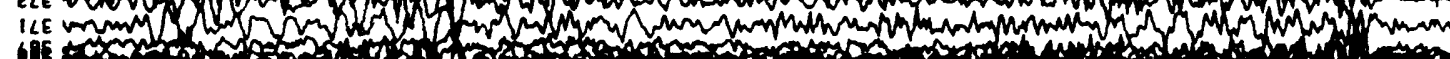
60

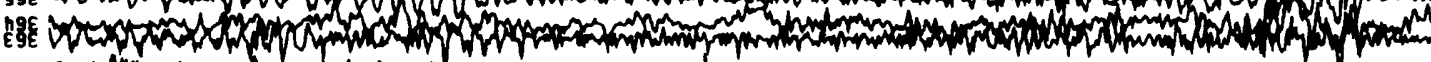

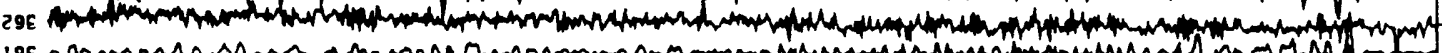

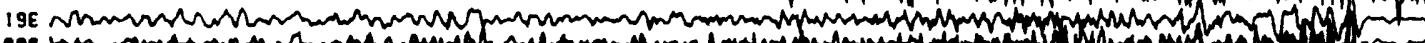

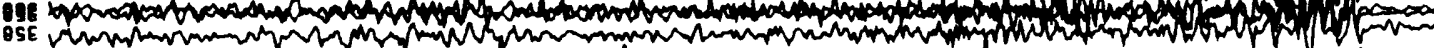

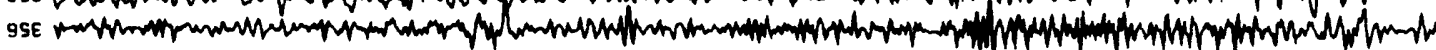

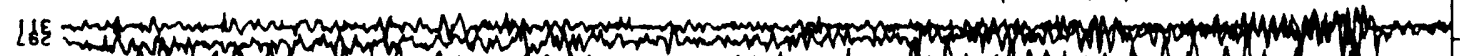
ISE OSE 2SE WWWMn

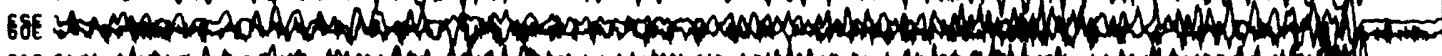

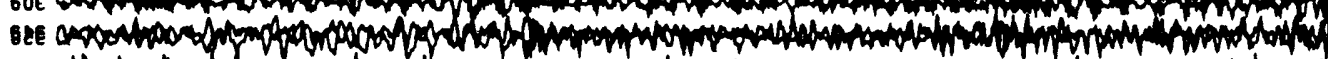

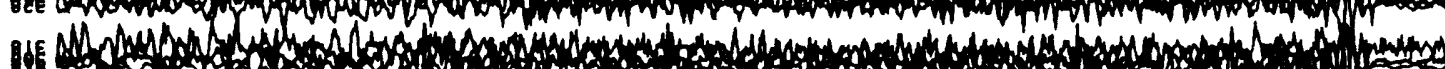
LE

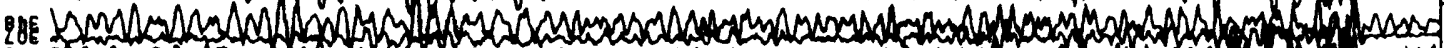
gie the

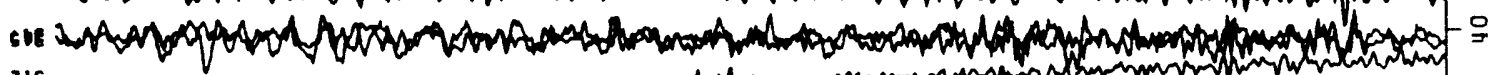

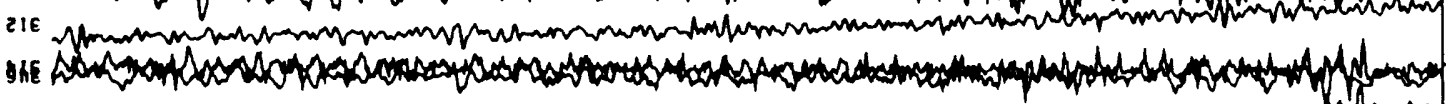
SOE

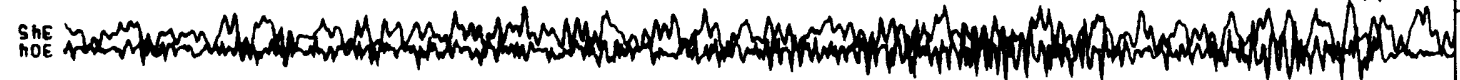

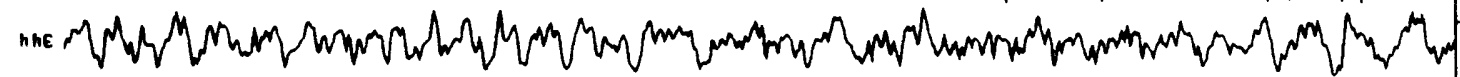

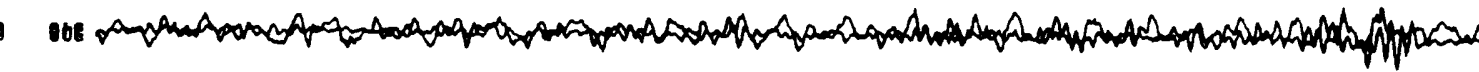

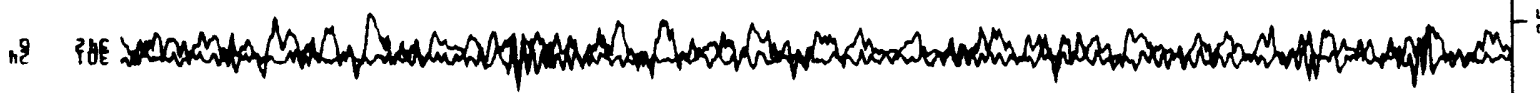

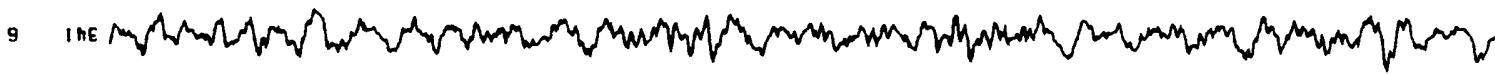

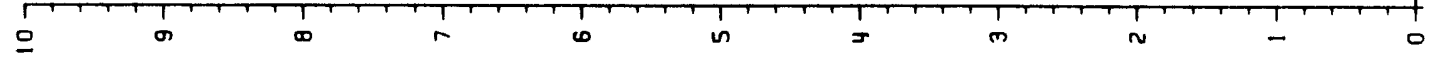




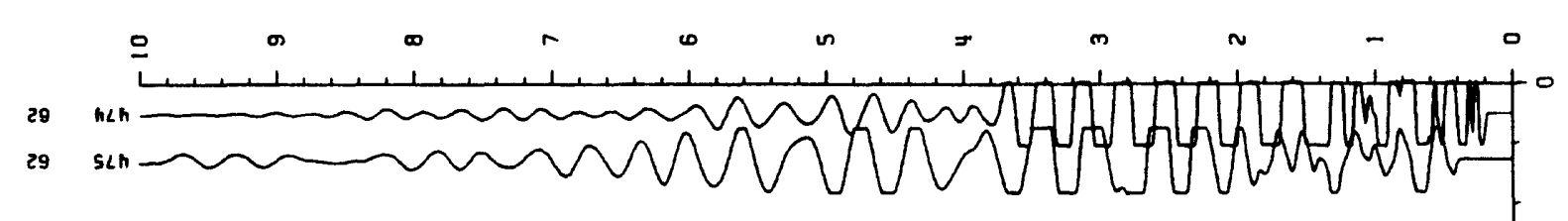

2h

(n)

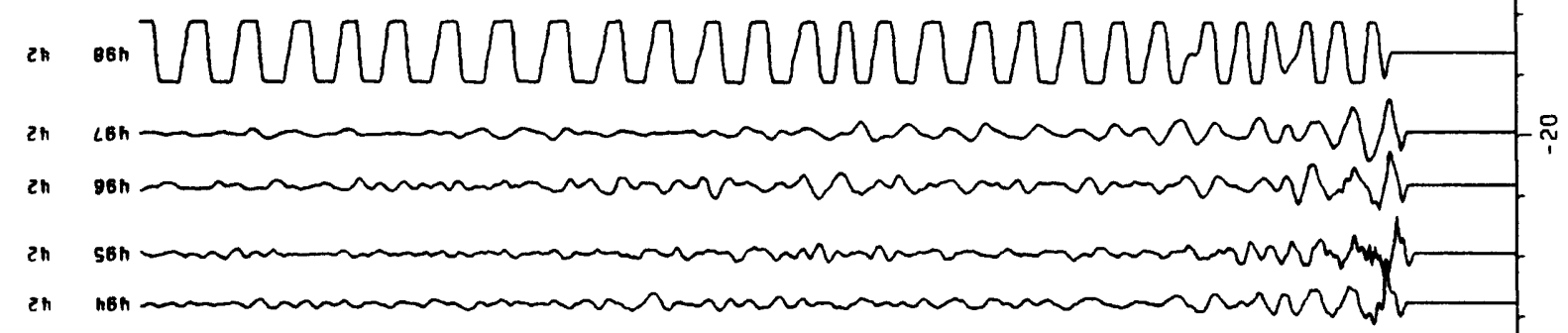

(s) ssh

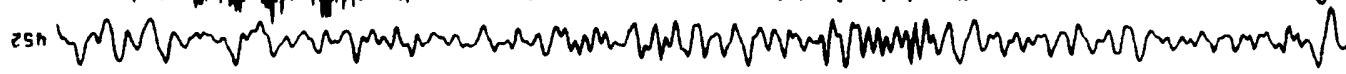

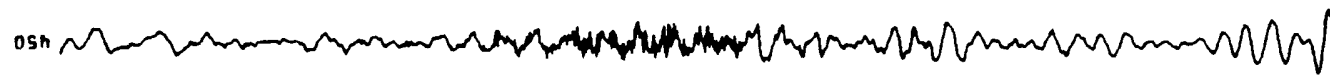
ons

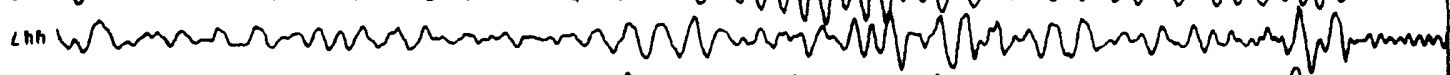

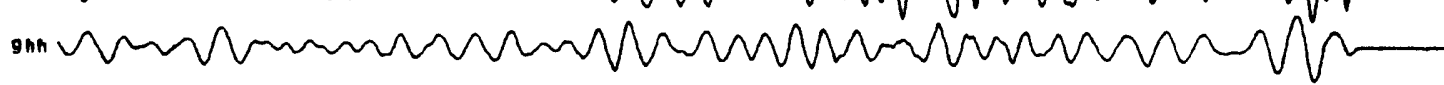

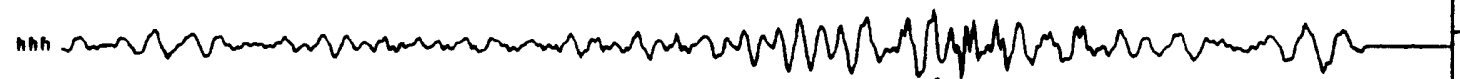

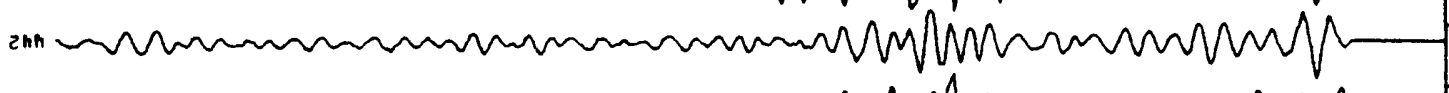
(T) 


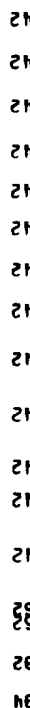

n6

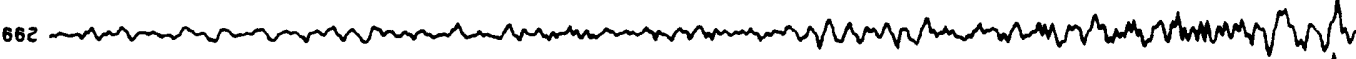
662 $600 \mathrm{C}$

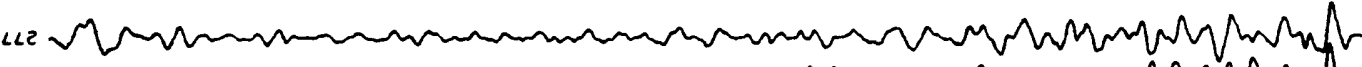
6ce

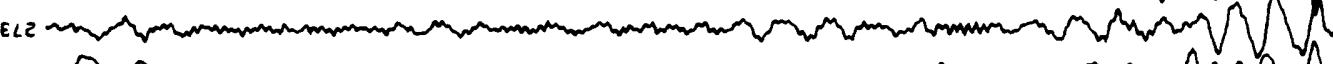

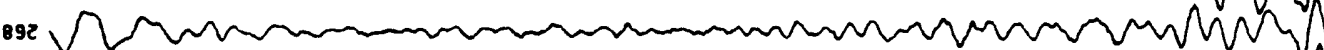
s92 29г (1) ${ }_{8 \mathrm{Fh}}=$ (12) O०2 W

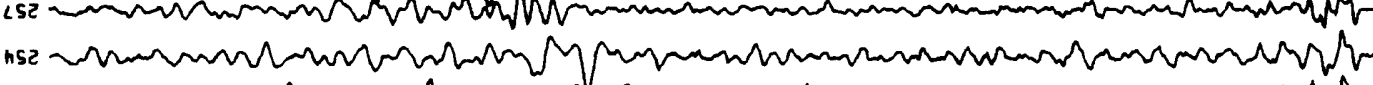

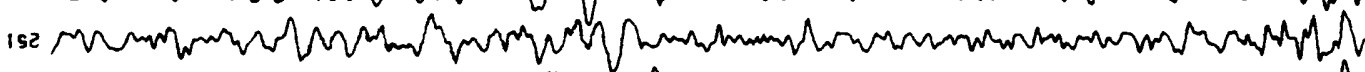

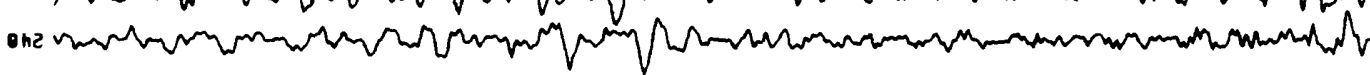

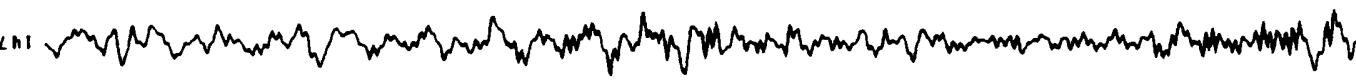

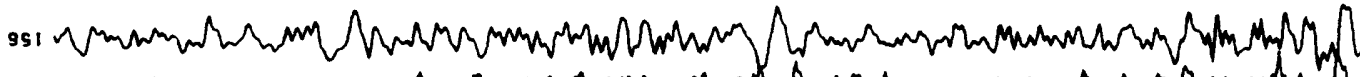

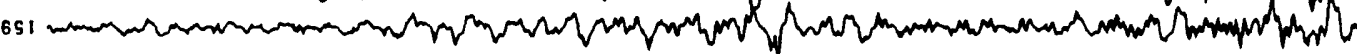$$
2 \pi n
$$

E८h
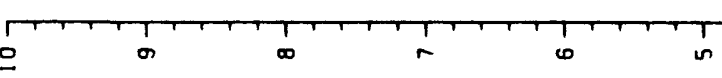

(1, 

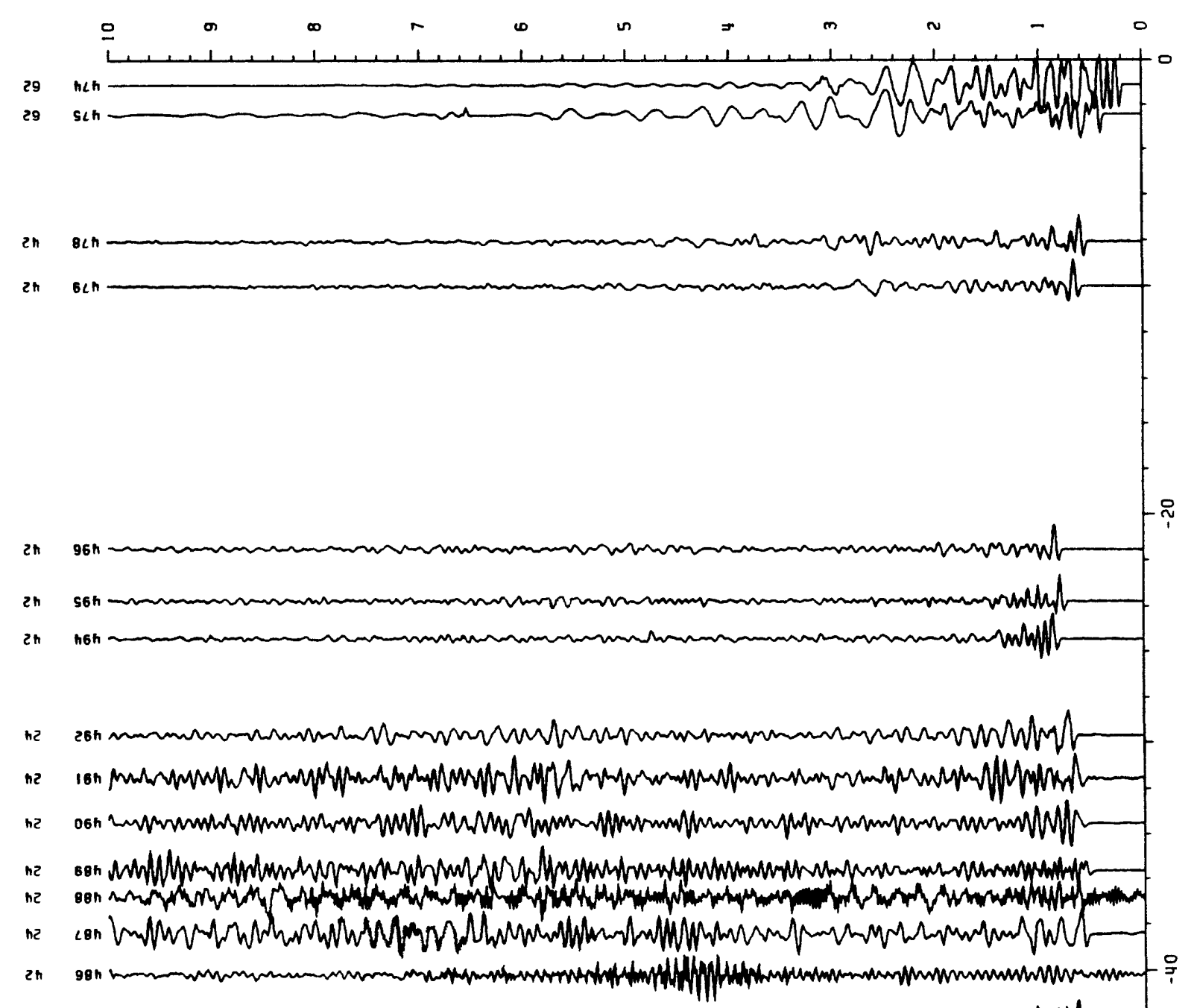

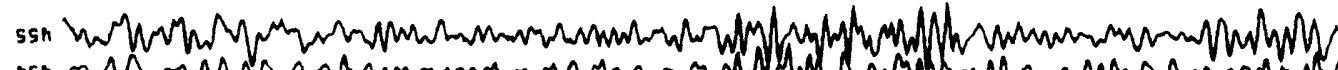

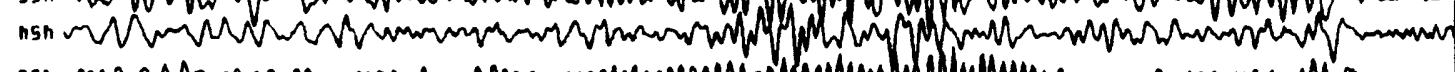

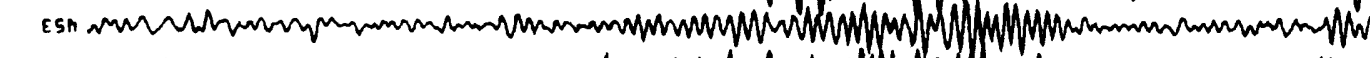

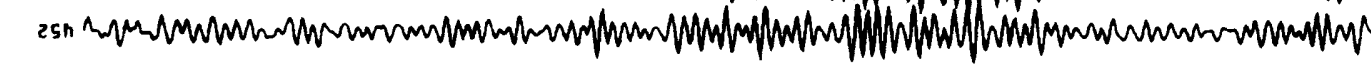

not com

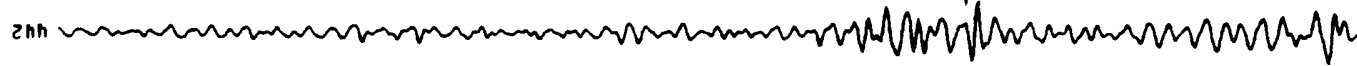

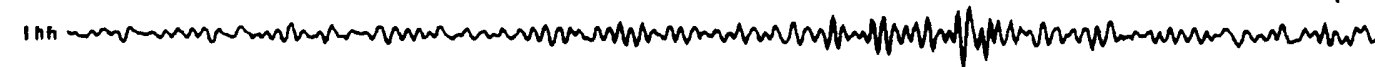

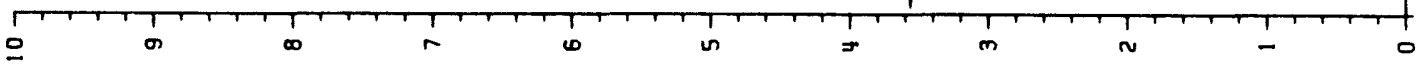


क

$\infty$

$\boldsymbol{\omega}$

in

$=$

$m$

N

- 0

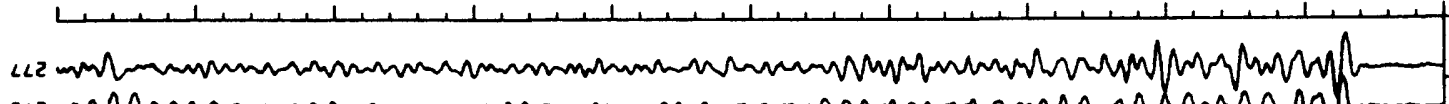

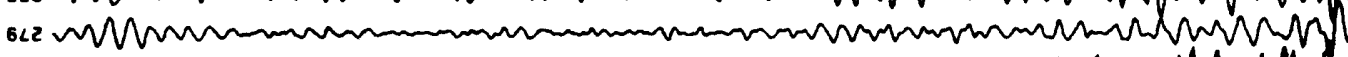
Ec2 s9z

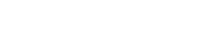

292 (n)

minn

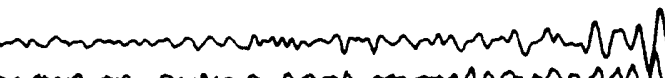

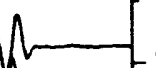

S22

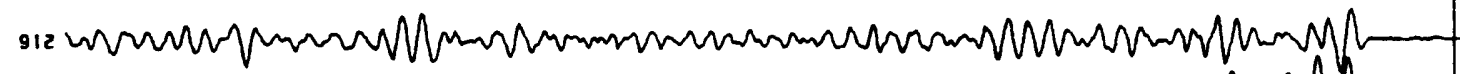

E12 w

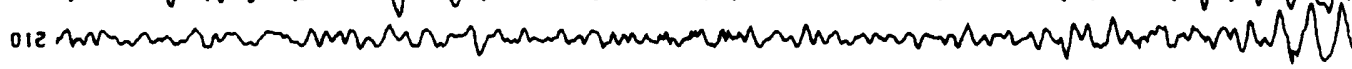

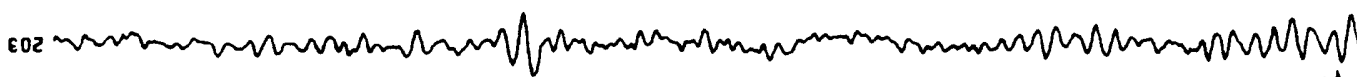
cse manthen

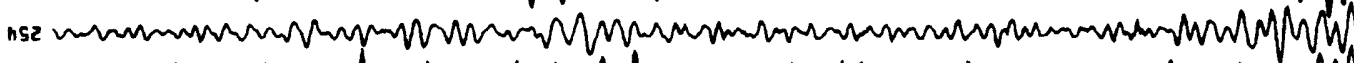

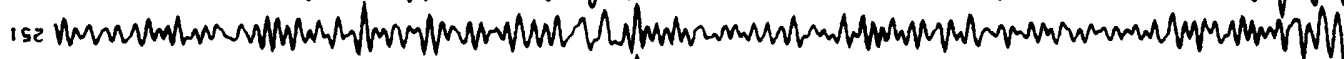

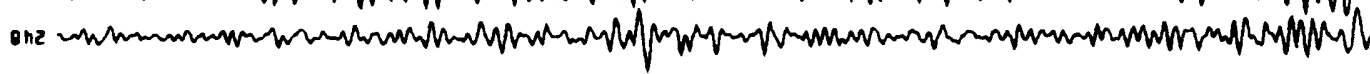

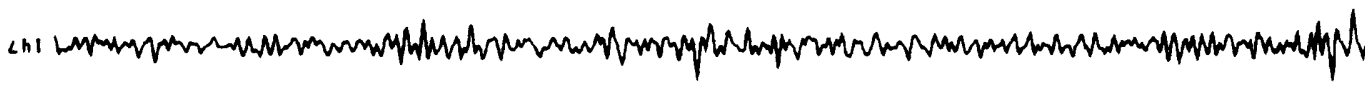

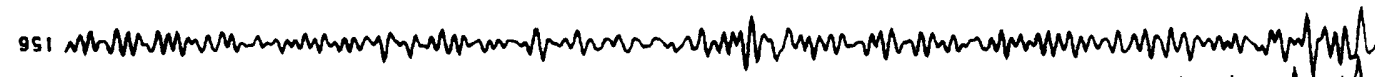

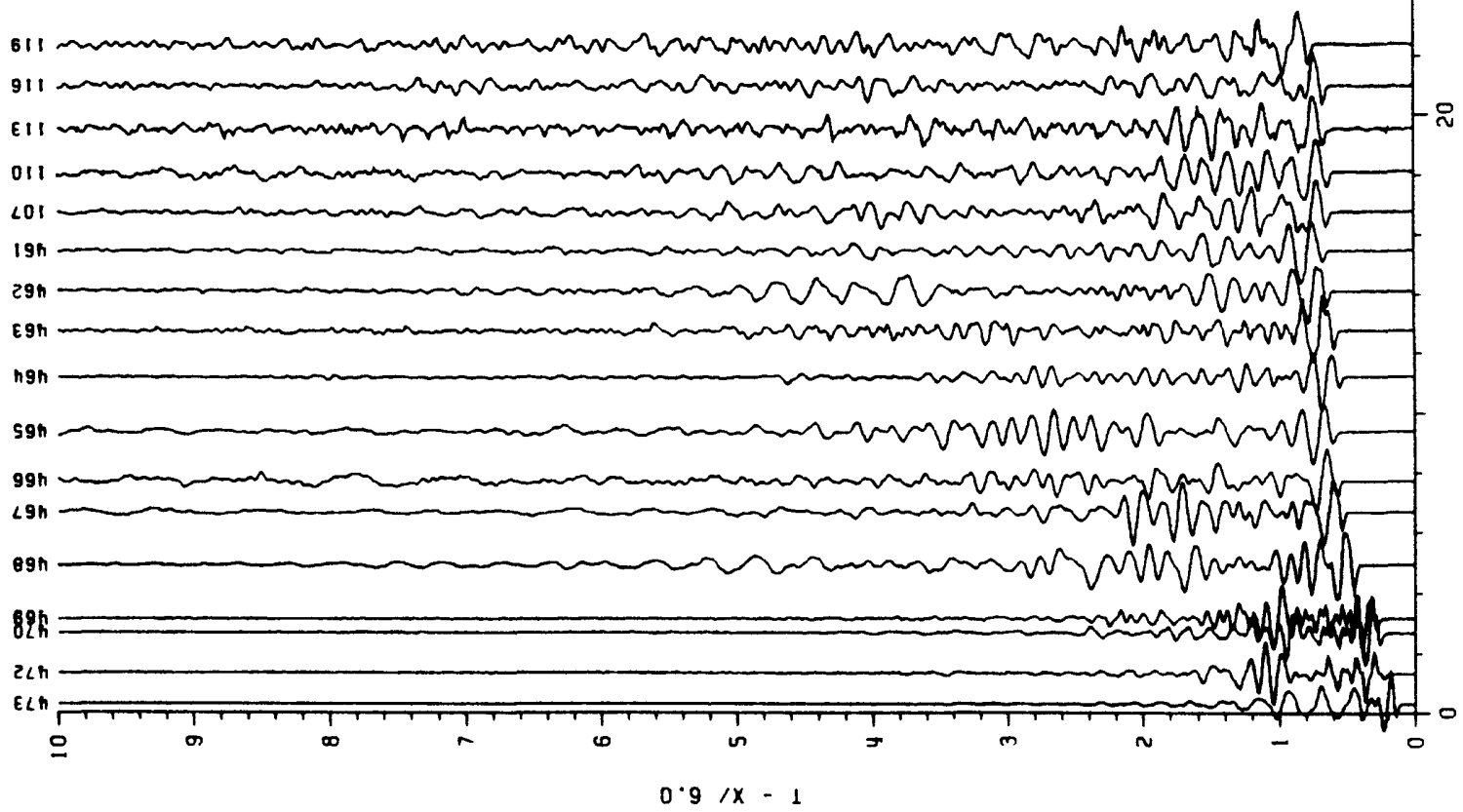




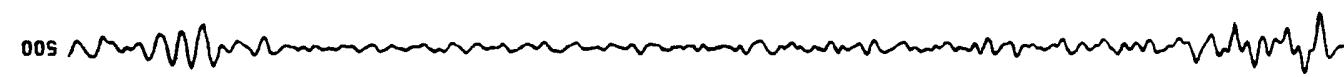

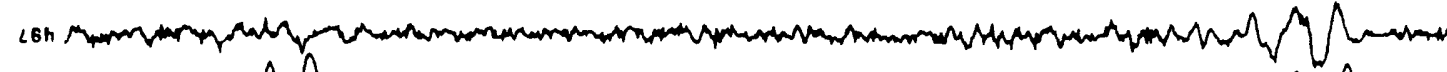

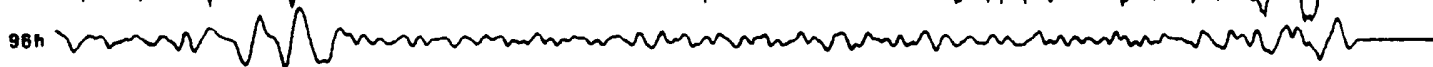

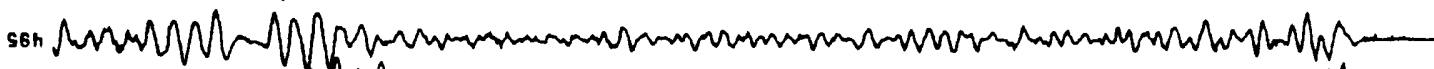

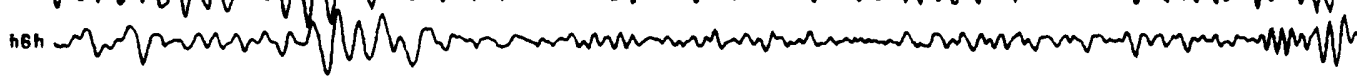

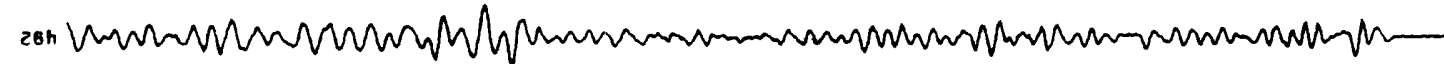

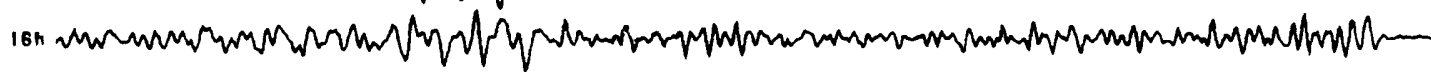

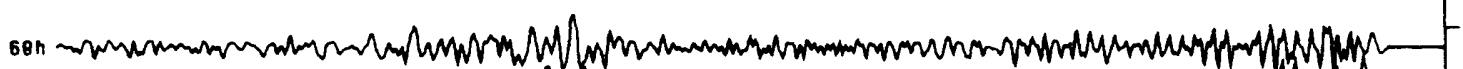

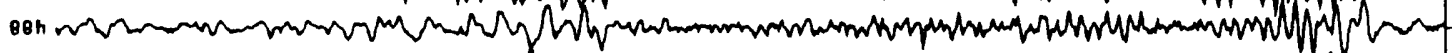
cos Wnomm

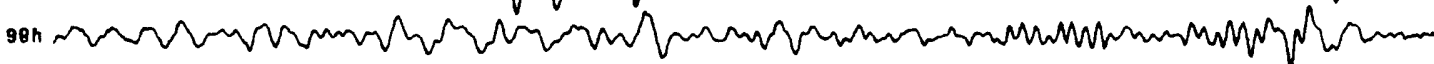

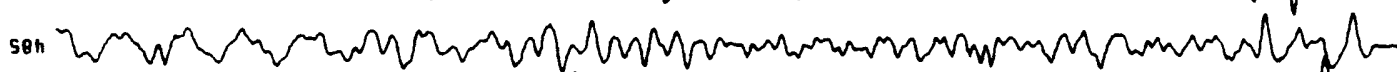
Beh

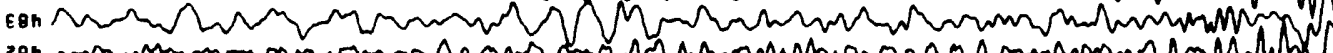
ces mom

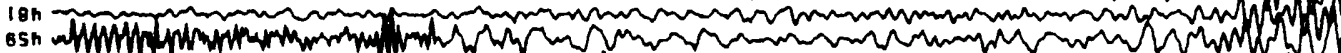
QSh

9sh ssh ESh 25h

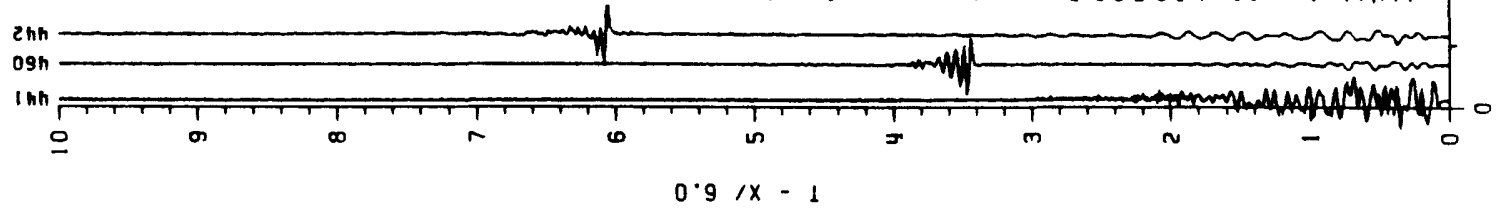




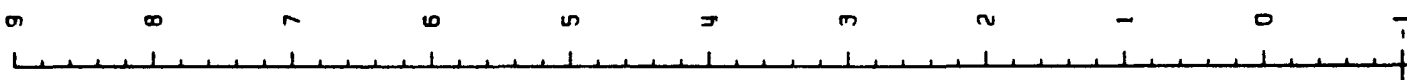

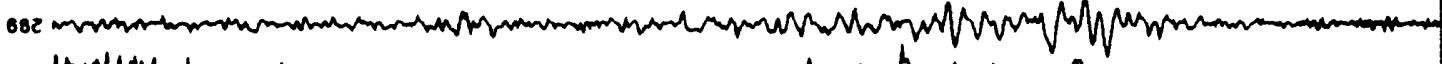

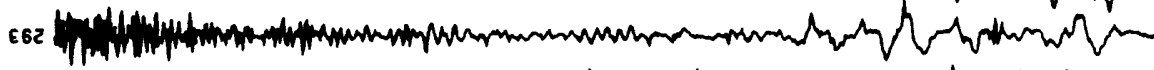

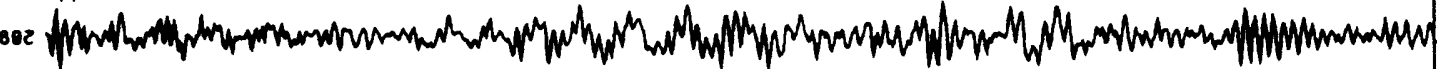
LL2 $8<2$

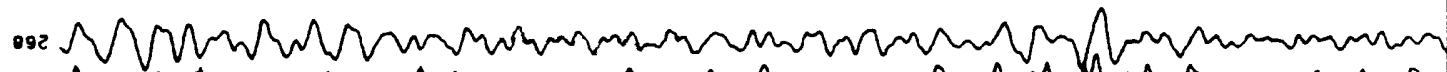

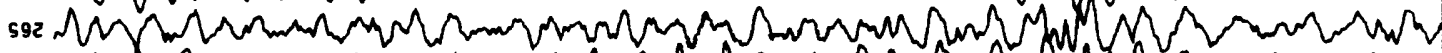

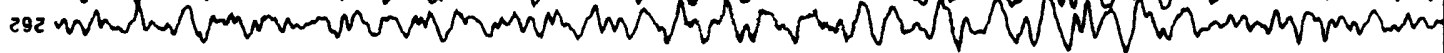

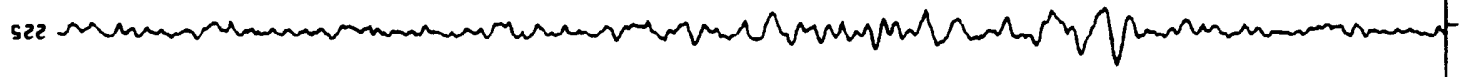

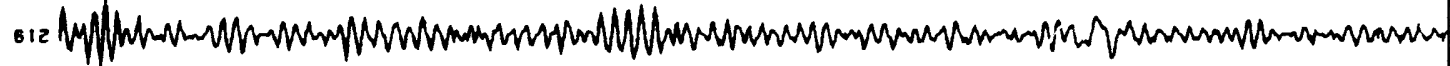

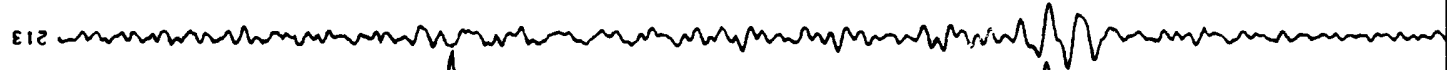
012 ${ }_{092}^{802}$ (a) cse ce

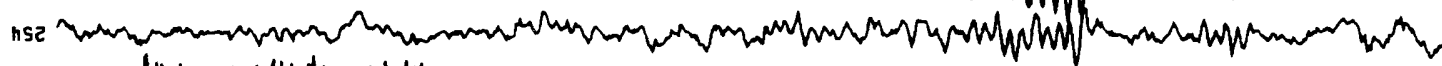

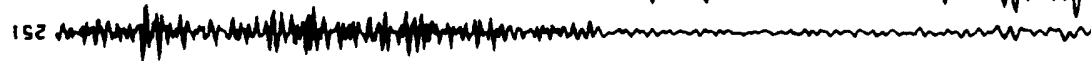
ohe nuyph

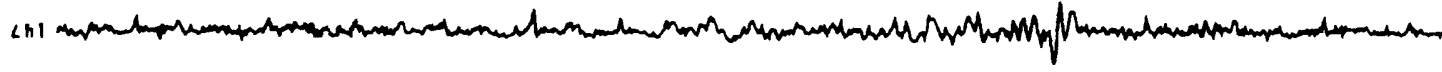

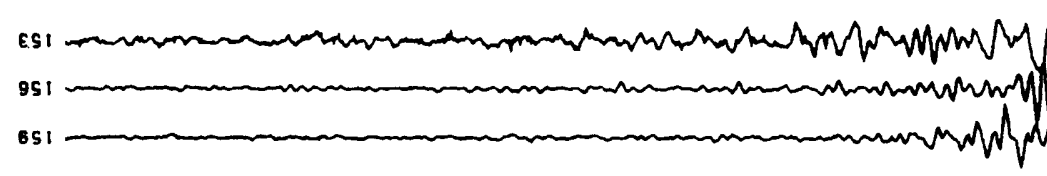

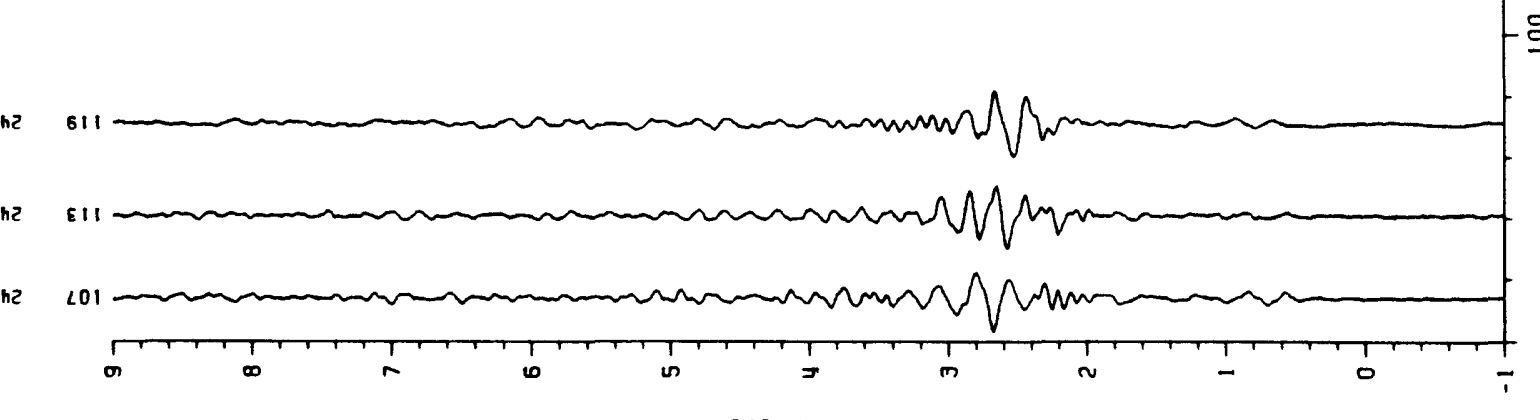


s62

${ }_{522}$

ols

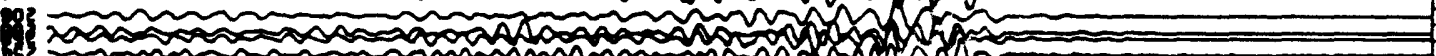

one war

ChI I

(5) 1 (1)

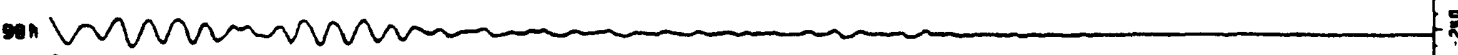

2t1
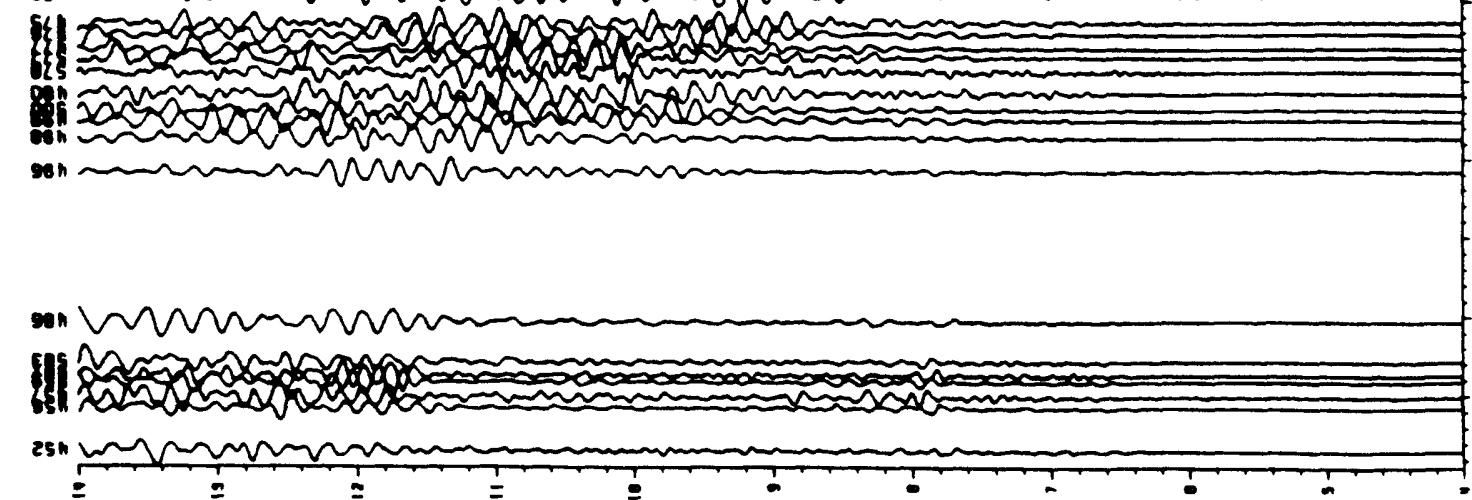

$0 \cdot 1 \times-1$ 